\title{
Surveying Business Uncertainty
}

\author{
David Altig, Jose Maria Barrero, Nicholas Bloom, \\ Steven J. Davis, Brent Meyer, and Nicholas Parker
}

\author{
Working Paper 2019-13c \\ June 2019 (revised August 2020)
}

\begin{abstract}
We elicit subjective probability distributions from business executives about their own-firm outcomes at a one-year look-ahead horizon. In terms of question design, our key innovation is to let survey respondents freely select support points and probabilities in five-point distributions over future sales growth, employment, and investment. In terms of data collection, we develop and field a new monthly panel Survey of Business Uncertainty (SBU). The SBU began in 2014 and now covers about 1,750 firms drawn from all 50 states, every major nonfarm industry, and a range of firm sizes. We find three key results. First, firm-level growth expectations are highly predictive of realized growth rates. Second, subjective uncertainty is highly predictive of forecast errors and the magnitude of future forecast revisions. Third, subjective uncertainty rises with the firm's absolute growth rate in the previous year and the extent of recent news about its growth prospects. We aggregate over firm-level forecast distributions to construct monthly indices of business expectations (first moment) and uncertainty (second moment) for the U.S. private sector.
\end{abstract}

JEL classification: L2, M2, 032, 033

Key words: business expectations, uncertainty, subjective forecast distributions, surveys

https://doi.org/10.29338/wp2019-13c

The authors are indebted to Mike Bryan, who played an instrumental role in launching the Survey of Business Uncertainty, to Emil Mihaylov for excellent research assistance, and to the authors' survey team: Grayson McAlister, Mea Resea Homer, Angelica Martini, Andres Carrillo-Rodriguez, Diana Basnakian, J., Alex Fields, Isabella Webber, Ethan Nadeau, Albert Hunecke, Mehak Ahmed, Paris Stroud, Luke Owens, Alexander Rangazas, J. Breuer, Nicholas Kogan, Daniel Brown, Brianna Goodrum, and Emilio Rodriguez. They also thank Tatsuro Senga for input about related Japanese surveys and the Federal Reserve Bank of Atlanta, the Alfred P. Sloan Foundation and the University of Chicago Booth School of Business for financial support. Finally, they thank our editor, Wilbert van der Klaauw, and two anonymous referees for many helpful comments that greatly improved the paper. The views expressed here are those of the authors and not necessarily those of the Federal Reserve Bank of Atlanta or the Federal Reserve System. Any remaining errors are the authors' responsibility. All results have been reviewed to ensure that no confidential information was disclosed.

Please address questions regarding content to David Altig, Federal Reserve Bank of Atlanta, dave.altig@atl.frb.org; Jose Maria Barrero, Instituto Tecnologico Autonomo de Mexico Business School, jose.barrero@itam.mx; Nicholas Bloom, Stanford University, nbloom@stanford.edu; Steven J. Davis, University of Chicago Booth School of Business and Hoover Institution, Steven.Davis@ChicagoBooth.edu; Brent Meyer, Federal Reserve Bank of Atlanta, brent.meyer@atl.frb.org; or Nicholas Parker, Federal Reserve Bank of Atlanta, nicholas.parker@atl.frb.org.

Federal Reserve Bank of Atlanta working papers, including revised versions, are available on the Atlanta Fed's website at www.frbatlanta.org. Click "Publications" and then "Working Papers." To receive e-mail notifications about new papers, use frbatlanta.org/forms/subscribe. 


\title{
Surveying Business Uncertainty
}

\author{
David Altig, ${ }^{1}$ Jose Maria Barrero, ${ }^{2}$ Nicholas Bloom, ${ }^{3}$ Steven J. Davis, ${ }^{4}$ \\ Brent Meyer ${ }^{1}$ and Nicholas Parker ${ }^{1}$
}

16 March 2020

\begin{abstract}
We elicit subjective probability distributions from business executives about their ownfirm outcomes at a one-year look-ahead horizon. In terms of question design, our key innovation is to let survey respondents freely select support points and probabilities in five-point distributions over future sales growth, employment, and investment. In terms of data collection, we develop and field a new monthly panel Survey of Business Uncertainty. The SBU began in 2014 and now covers about 1,750 firms drawn from all 50 states, every major nonfarm industry, and a range of firm sizes. We find three key results. First, firm-level growth expectations are highly predictive of realized growth rates. Second, firm-level subjective uncertainty predicts the magnitudes of future forecast errors and future forecast revisions. Third, subjective uncertainty rises with the firm's absolute growth rate in the previous year and with the magnitude of recent revisions to its expected growth rate. We aggregate over firm-level forecast distributions to construct monthly indices of business expectations (first moment) and uncertainty (second moment) for the U.S. private sector.
\end{abstract}

Keywords: Business Expectations, Uncertainty, Subjective Forecast Distributions, Surveys

JEL Classification: L2, M2, O32, O33.

Disclaimer: Any opinions and conclusions expressed herein are those of the authors and do not necessarily represent the views of the Federal Reserve Bank of Atlanta. All results have been reviewed to ensure that no confidential information was disclosed.

Acknowledgements: We are indebted to Mike Bryan, who played an instrumental role in launching the Survey of Business Uncertainty, to Emil Mihaylov for excellent research assistance, and to our survey team: Grayson McAlister, Mea Resea Homer, Angelica Martini, Andres CarrilloRodriguez, Diana Basnakian, J., Alex Fields, Isabella Webber, Ethan Nadeau, Albert Hunecke, Mehak Ahmed, Paris Stroud, Luke Owens, Alexander Rangazas, J. Breuer, Nicholas Kogan, Daniel Brown, Brianna Goodrum, and Emilio Rodriguez. We thank Tatsuro Senga for input about related Japanese surveys and the Federal Reserve Bank of Atlanta, the Alfred P. Sloan Foundation and the University of Chicago Booth School of Business for financial support. Finally, we thank our editor, Wilbert van der Klaauw, and two anonymous referees for many helpful comments that greatly improved the paper.

${ }^{1}$ Federal Reserve Bank of Atlanta, ${ }^{2}$ Instituto Tecnológico Autónomo de México Business School, ${ }^{3}$ Stanford University, ${ }^{4}$ University of Chicago Booth School of Business and Hoover Institution 


\section{Introduction}

Uncertainty is a fundamental fact of economic life. Businesses and households grapple with uncertainty in forming plans and making decisions. The extent and nature of uncertainties change over time, sometimes gradually and sometimes abruptly, altering the outlook for decision makers and affecting their choices. Recent history offers some vivid examples: the $9 / 11$ terrorist attacks, the Global Financial Crisis, banking and sovereign debt crises in the Eurozone, the June 2016 Brexit referendum, a dramatic escalation of trade policy tensions under the Trump Administration, and the coronavirus pandemic of 2020. These examples underscore the need for sound, flexible measures of uncertainty, so that we can better understand and model the relationship of perceived uncertainty to economic decisions, outcomes, and performance.

We would like to track the uncertainty that agents perceive in their external environments and the uncertainty they perceive about own future outcomes, e.g., a firm's future sales. A standard approach maintains rational expectations and some form of stationarity, so that past conditional volatility can serve as the basis for inferences about uncertainty over future outcomes. Examples include Bloom (2009), Fernández-Villaverde et al. (2011), Jurado, Ludvigson, and Ng (2015), and Colacito et al. (2018). Another approach treats the dispersion in point forecasts as a proxy for uncertainty (e.g., Bachmann, Elstner and Sims, 2013). Scotti (2016) uses surprises in economic data releases to proxy for uncertainty. Yet another approach relies on newspapers and other text sources to construct uncertainty measures, as in Baker, Bloom, and Davis (2016), Handley and Li (2018) and Hassan et al. (2019). Datta et al. (2017) offer an extensive overview of various approaches, with a focus on measuring uncertainty in the external environment.

While valuable, these approaches may not adequately capture the subjective uncertainty that agents perceive, which presumably is what drives their decisions. There is a now-large body 
of evidence that subjective expectations deviate systematically from the expectations implied by rational expectations with full use of available information. ${ }^{1}$ In addition, many of the most prominent empirical proxies for uncertainty pertain to distinct theoretical concepts and differ in their statistical properties (Kozeniauskas et al., 2018). These observations argue for a measurement approach that gets directly at the uncertainty agents perceive without invoking assumptions about rationality, information, and stationarity.

We - a group of researchers at the Atlanta Fed, Chicago Booth and Stanford - set out in 2013 to develop and field a new survey instrument to measure the perceived uncertainty of senior decision makers in U.S. firms. In doing so, we built on earlier work that elicits subjective beliefs from households, as in Dominitz and Manski (1997) and Manski (2004). ${ }^{2}$ We spent about a year on initial field testing of various question designs, conducting cognitive interviews, and creating the Survey of Business Uncertainty (SBU). Since 2014, the SBU has collected subjective probability distributions over own-firm future outcomes from a panel of business executives. We send them surveys each month and recruit new firms over time, with the aim of collecting long response histories for many firms. As of October 2019, we have data for 1,743 firms drawn from all 50 states, every major nonfarm industry, and a wide range of firm sizes.

\footnotetext{
${ }^{1}$ Examples include Coibion and Gorodnichenko (2012, 2015) and Bordalo et al. (2019) for professional forecasters, Malmendier and Tate (2005), Ben-David, Graham, and Harvey (2013), Gennaioli, Ma and Shleifer (2016) and Barrero (2020) for firm managers, Barber and Odean (2001), Bailey et al. (2011), Puetz and Ruenzi (2011) and Akepanidtaworn et al. (2019) for investors and mutual fund managers, and Roszypal and Schlaffmann (2017) for consumers.

${ }^{2}$ Manski (2004) is an early advocate of measuring subjective expectations by asking survey respondents to assign probabilities to pre-specified outcomes. Most of this work surveys households and consumers. The University of Michigan Survey of Consumers (www.sca.isr.umich.edu) has long asked households to assign probabilities to binary outcomes defined over family income, job loss, inflation, and more. The New York Fed's Survey of Consumer Expectations (www.newyorkfed.org/microeconomics/sce) includes questions with a similar structure and questions that elicit probabilities over multiple prespecified outcomes, e.g., bins defined by inflation rate intervals. See Armantier et al. (2017).
} 
Our core survey questions elicit five-point subjective probability distributions over each firm's own future sales growth, employment, and capital expenditures. The look-ahead horizon is four quarters or twelve months, depending on the outcome variable. Survey respondents freely select five support points and then assign probabilities to each. This approach affords great flexibility for the respondent, allowing for high or low expected growth, uncertain or predictable outlooks, and negative or positive skew in the distribution over future outcomes. It also avoids anchoring, because our question format specifies neither the location nor spread of the support points. Respondents nearly always update their subjective distributions across consecutive surveys, usually by modest amounts. This result suggests that they are attentive to the survey and actively updating their responses as their perceptions change over time.

Using the subjective probability distributions, we measure expected future outcomes and the uncertainty surrounding those outcomes for each firm. Since the SBU includes questions about past and current outcomes, we can readily relate subjective forecast distributions to realized outcomes. Growth rate expectations are highly predictive of realized growth rates in the firm-level data, even after conditioning on firm and time fixed effects. Subjective uncertainty is highly predictive of absolute forecast errors. In addition, when firms express greater uncertainty about future outcomes, they make larger forecast revisions in the future. So, what drives subjective uncertainty? We show that it exhibits a pronounced V-shaped relationship to the firm's recent past growth, echoing similar results in Bachmann et al. (2018) and Bloom et al. (2017). Exploiting the panel dimension of the SBU, we also show that subjective uncertainty rises with the size of the firm's most recent forecast revision. Barrero (2020) provides additional evidence on the properties of the subjective probability distributions in SBU data, which we summarize in Section 3. 
We also use SBU data to construct time series of the cross-firm average subjective expectations of employment growth, sales growth, and investment rates and the corresponding average subjective uncertainty levels. We began publishing these indices in November 2018 at www.frbatlanta.org/research/surveys/business-uncertainty, and they are now carried by Haver Analytics, Bloomberg, and the St. Louis Fed's FRED database (http://fred.stlouisfed.org).

The SBU differs from earlier surveys of beliefs and expectations in key respects: an innovative question design for eliciting subjective probability distributions, a focus on outcomes at the respondent's own firm, a monthly sampling frequency, and broad coverage of the U.S. nonfarm private sector. For example, the quarterly Duke CFO Survey elicits perceptions of aggregate uncertainty in the form of 80 percent confidence intervals for future S\&P 500 returns and, more recently, for U.S. GDP growth. ${ }^{3}$ See Ben-David, Graham, and Harvey (2013) and cfosurvey.org. Surveys in Germany and Japan collect data on the expectations of firm-level variables. See Bachmann and Elstner (2015), Massenot and Pettinichi (2018), Tanaka et al. (2019) and Chen et al. (2019). While these surveys do not elicit subjective probability distributions, the ifo Business Tendency Survey collects quarterly data on the best- and worst-case sales growth scenarios of German firms (Bachman et al., 2018). The closest forerunner to the SBU is the Bank of Italy's Survey on Investment in Manufacturing, which has elicited subjective probability distributions at an annual frequency for decades (Guiso and Parigi, 1999). The SBU is also closely related to the Atlanta Fed's monthly Business Inflation Expectations (BIE) Survey. We conducted our initial field testing of SBU questions as part of the BIE's special question series.

\footnotetext{
${ }^{3}$ In 1947, the U.S. Department of Commerce and the Securities and Exchange Commission began fielding a quarterly survey that elicited point forecasts of firm-level sales and capital expenditures (Friend and Bronfenbrenner, 1955). The survey evolved over time, migrated to the Census Bureau, and ended in 1996 for budgetary reasons. The Empire State Manufacturing Survey has elicited forecast densities for firms' input price changes since 2009. See, for example, Federal Reserve Bank of New York (2013).
} 
Although a young survey, the SBU approach to eliciting subjective probability distributions from business managers has already been adopted in several other surveys with largescale institutional backing. The U.S. Census Bureau put questions with the SBU design to about 50,000 manufacturing plants as part of the Management and Organizational Practices Survey (Bloom et al., 2017). Since August 2016, the Bank of England and University of Nottingham have fielded a monthly U.K. Decision Maker Panel Survey that follows the SBU closely, and which has proved especially useful in assessing business expectations and uncertainty related to Brexit (Bloom et al., 2018a). The British Office for National Statistics put questions that follow the SBU design to about 25,000 firms in 2017 as part of the new U.K. Management and Expectations Survey (Awano et al., 2018). Statistical agencies in China and Japan have also developed and fielded surveys of business managers that incorporate the SBU question design for eliciting subjective probability distributions over own-firm and aggregate outcomes. ${ }^{4}$

We enhance the value of the SBU by collecting additional information from our survey participants alongside our core data on past, current, and future outcomes. Special questions each month elicit (a) subjective probability distributions over other firm-level or aggregate outcomes, (b) information about the firm's characteristics or information processes, or (c) the perceived effects of specific economic and policy developments on the firm's own outcomes. In February 2018, for example, we asked whether and how the 2017 Tax Cut and Jobs Act caused firms to revise their capital investment plans for 2018 and 2019. In 2019, we posed several questions about

\footnotetext{
${ }^{4}$ The China Employer-Employee Survey (CEES) fielded SBU-type questions to 1,700 manufacturing firms in 2018 and is slated to gather the corresponding realizations in 2020. See Section 2 in Bloom et al. (2018b) for a description of the CEES. The Social Research Institute of Japan put three-point versions of the SBU questions to managers at about 13,600 manufacturing plants in 2017 and is now planning a second wave. Japan's Research Institute of Economy, Trade and Industry used the SBU question design in a 2017 survey to elicit subjective probability distributions over own-firm and aggregate outcomes. These Japanese surveys are not yet the subject of a paper in circulation, to our knowledge.
} 
the past and prospective effects of trade policy developments on investment, employment and sales. Aggregating over the firm-level responses to these questions yields survey-based estimates for the causal effects of policy developments. See Altig et al. (2019bc).

Section 1 describes the SBU and our approach to eliciting subjective forecast distributions. Section 2 explains how we measure firm-level expectations, uncertainty, and forecast errors. Section 3 relates subjective beliefs to future outcomes, forecast errors, and past outcomes. It also provides information about how firms update their beliefs over time. Section 4 presents activityweighted average measures of business expectations and uncertainty. Section 5 presents additional results, including evidence that the shape of SBU subjective forecast distributions has predictive value for realized growth rates and the sign of forecast errors.

\section{The Survey of Business Uncertainty}

\section{A. Core Question Design}

The SBU elicits subjective probability distributions from business executives about ownfirm future outcomes. To fix ideas, consider a discrete probability distribution over, say, the future sales growth rates of a firm. Suppose the distribution has $N$ support points, $\left.\{\text { SaleG }\}_{i}\right\}_{i=1}^{N}$, with associated probabilities $\left\{p_{i}\right\}_{i=1}^{N}$. Given survey response values for these support points and probabilities, we can calculate the respondent's (mean) expectation of the sales growth rate as

$$
\operatorname{Mean}(\text { SaleGr })=\sum_{i=1}^{N} p_{i} \cdot \operatorname{SaleG} r_{i}
$$

and his or her subjective uncertainty as the standard deviation,

$$
S D(\text { SaleGr })=\left[\sum_{i=1}^{N} p_{i}\left(\text { SaleGr }_{i}-\text { Mean }(\text { SaleGr })\right)^{2}\right]^{1 / 2} .
$$

Of course, we don't know how respondents conceptualize future growth rate possibilities. They may think in terms of fewer or more support points, or in terms of a continuous distribution. 
Subjective distributions are also likely to differ greatly across respondents in terms of location, scale, and shape and perhaps over time for individual firms. These observations argue for a question design that gives much flexibility to the respondent. In this regard, note that discrete distributions with few support points are highly flexible. A distribution with $N=5$ allows nine degrees of freedom (since the probability values sum to 1), more than enough to approximate most common parametric distributions. It also accommodates symmetric and asymmetric, single- and multi-mode, thin and fat-tailed distributions and those with wide or narrow support.

Several other considerations figure in our thinking about SBU question design. First, we require questions that respondents can comprehend and answer without undue burden. Much of our field testing and early analysis of survey responses focused on comprehension, as discussed in (Online) Appendix B. In addition, we conducted face-to-face cognitive interviews with small groups of SBU panel members (4-6 respondents per group), which also helped us assess comprehension. Second, business executives place a high value on their time. Thus, we aim for a short survey instrument with an average completion time of about five minutes. To help meet this goal, we split the panel into three groups, each of which rotates through the full set of core questions every three months. ${ }^{5}$ One group gets the employment questions in any given month, one gets the sales questions, and one gets the investment questions. Third, the SBU is a selfadministered, web-based survey, which requires questions that elicit answers without intervention by an enumerator or other survey representative.

These considerations led us to a survey instrument in which respondents freely assign values to five discrete support points and then assign probabilities to each. Figure 1a and $1 \mathrm{~b}$ display

\footnotetext{
${ }^{5}$ Early on, we split the panel into two groups and asked more questions each month. We shifted to the three-group design in May 2019 to maintain short response times. See Appendix B for details.
} 
the core SBU employment and sales growth questions. Appendix A displays the investment questions. For each topic, the survey first asks for the current outcome in levels. Next, it asks about recent past outcomes - e.g., employment twelve months ago or the sales growth rate over the past twelve months. Then it asks the respondent to specify five future outcomes, ranked from lowest to highest, looking twelve months ahead for employment and from the current quarter to four quarters hence for the sales growth rate. Finally, the survey elicits probabilities for each of the five respondent-provided support points on the subjective distribution.

A series of field tests and cognitive interviews before launching the SBU revealed that business decision makers are willing and able to express beliefs about their firm's outlook in terms of discrete probability distributions with freely chosen support points. We began a new round of cognitive interviews in late 2019 to gather information about forecasting methods in use by our panelists and to solicit their thoughts about our survey instrument. Forecasting methods vary across firms but typically rely on some combination of the firm's sales history, conversations with key customers about anticipated product demand, and attention to industry trends and policy developments that could affect demand or costs. Interviewees report little difficulty in answering our forecast distribution questions, even when their internal forecasting methods do not parallel our question design. This pattern fits with longstanding evidence that consumers can express uncertainty about future events using subjective probabilities (e.g., Manski, 2004).

Our approach accords well with how business managers are taught to conceptualize uncertain future outcomes. To document this claim, we reviewed three top-selling textbooks in corporate finance, a subject with heavy enrollments in business schools. Nearly 75 percent of the examples and exercises about risk or uncertainty in these books specify discrete scenarios or 
probability distributions to formalize uncertainty. ${ }^{6}$ Since more than 70 percent of our panel members are CEOs, CFOs, or have some other finance-related title, most are likely to be comfortable conceptualizing uncertainty in a manner that relates easily to our question design.

Many surveys that elicit subjective probability distributions over future economic outcomes use pre-specified bins or support points, as in the Philadelphia Fed's Survey of Professional Forecasters. That approach may work well when survey designers and respondents have a common understanding about the plausible range of outcomes for the variable of interest, say GDP or the firm's input costs. However, it is ill-suited for forecasts of firm-level growth rates, given the large differences in the central tendency and dispersion of their growth rates. ${ }^{7}$ Our approach also avoids anchoring effects associated with pre-specified ranges or support points. By allowing free choice of support points and probabilities, we also let the respondent determine the shape of the forecast distribution. In contrast, Bachmann et al. (2018) create subjective distributions by imposing a triangular shape from "lowest" to "highest" scenarios.

Ours is not the only question design that gives flexibility to the respondent, accommodates great heterogeneity in subjective distributions, and avoids anchoring. The "unfolding brackets" approach presents survey respondents with a sequence of questions to elicit quantiles of the

\footnotetext{
${ }^{6}$ The three textbooks are Principles of Corporate Finance by Richard A. Brealey, Stewart C. Myers; Corporate Finance by Stephen Ross, Randolph Westerfield, Jeffrey Jaffe and Bradford Jordan; and Corporate Finance by Jonathan Berk and Peter DeMarzo. The second most-common approach uses a parametric distribution with one or two parameters, which is not flexible enough for our purposes. ${ }^{7}$ Caves (1998) and Davis and Haltiwanger (1999) review and add to an extensive literature documenting large differences in the central tendency and dispersion of business growth rates by industry, size, and age. Bloom et al. (2017) show that managers' expectations and subjective uncertainty fall sharply with size and age and rise with the past volatility of the establishment, its parent firm, and its industry. In principle, a survey designer could condition on all these factors to tailor pre-specified bins that vary by firm and time. Even when rich data of this sort are available, however, senior executives have more information about own-firm growth prospects and risks than survey designers. Moreover, it is unclear how a survey designer should adjust bins in the wake of unusual or extraordinary shocks, e.g., the 2020 coronavirus pandemic. Letting respondents freely select support points and probabilities respects firmspecific information without requiring that survey designers possess such information.
} 
subjective distribution. For example, one can first pose a question that elicits the median of the subjective distribution over the growth rate of future sales, then pose two questions to elicit the $75^{\text {th }}$ and $25^{\text {th }}$ percentiles, and so on. See, for example, Juster and Suzman (1995) and Hurd (1999).

Relative to unfolding brackets, our approach offers two advantages. First, it yields a shorter survey instrument with fewer questions. Eliciting five quantiles via unfolding brackets requires a sequence of five questions, whereas our design elicits a five-point distribution in two questions. Given our respondents are senior executives, a longer survey would tax their patience further and likely lower response rates and sample retention. Second, as noted above, our approach aligns well with how managers are taught to conceptualize uncertainty in terms of scenario planning.

We close our discussion of question design with a detail that has important effects on response accuracy. Our forward-looking questions elicit beliefs about the level of future employment but the growth rate of future sales. Respondents tend to think in these terms. Moreover, field testing revealed that asking about the level of future sales yields more response errors of two types: adding or dropping a digit when entering values for support points, and the inconsistent use of units across surveys - or even in the same survey. For example, a respondent might switch from quarterly to annual sales or thousands to millions of dollars. While we developed methods to detect and correct these sorts of response errors, we also experimented with question formulation to reduce the incidence of such errors. As of September 2016, we settled on a formulation that asks about past and future sales growth rates and the level of sales in the current quarter. Appendix A presents earlier incarnations of our sales-related questions.

\section{B. Sampling, Panel Recruitment, and Response Rates}

We obtain lists of randomly selected firms and their senior executives from an affiliate of Dunn \& Bradstreet, a supplier of business information and research. In turn, we sample from these 
lists to recruit panel members, working with a team of research assistants at the Atlanta Fed. We aim for a panel of firms that is reasonably well balanced across industries and regions. We deliberately oversample larger firms and, to a lesser extent, firms in cyclically sensitive industries. The recruitment process continues, as we build and refresh the SBU panel over time. Each month, we deliver the survey link to panel members via email and let them fill it out over a two-week period on their own time.

During the period from June 2014 to June 2018, approximately 42 percent of potential contacts reached via telephone agreed to join the panel. Among those who joined, 62 percent responded to the survey at least once. In any given month, about 43 percent of continuing panel members respond to the survey. ${ }^{8}$ These high response rates in a voluntary survey of business executives reflect the resources we devote to sample recruitment and maintenance. As of AugustOctober 2019, we receive about 360 completed survey responses per month. The median survey completion time is 4.4 minutes, and the mean is 7.6 minutes. $^{9}$ See Appendix A for more information about recruitment and response rates.

\section{Survey Development, Testing, Data Cleaning, and Sample Mix}

We began fielding trial SBU questions in October 2013 as part of the Atlanta Fed's monthly Business Inflation Expectations (BIE) Survey, which samples firms in the Sixth Federal Reserve District (Florida, Georgia, Alabama, and parts of Tennessee, Mississippi, and Louisiana). In July 2014, we launched the SBU as a separate national survey, originally known as the Decision Maker

\footnotetext{
${ }^{8}$ These response rates refer to the period from September 2016 (the last major change in core survey questions) to October 2018.

${ }^{9}$ These statistics pertain to the period since May 2019, when we began asking about only one core topic (sales, employment, or investment) per panel group per month. Median and mean survey completion times before May 2019 are 5.5 and 8.7 minutes, respectively. In computing these statistics, we winsorize completion times at the $90^{\text {th }}$ percentile to deal with respondents who open the survey tool and set it aside for a spell (possibly days) before returning to the tool and completing their responses.
} 
Survey. Over the past six years, we experimented with several aspects of our question design: preselected support points, fewer support points, interval bins in place of support points, fixed probabilities for respondent-chosen support points or bins, and other matters. We also fielded questions that elicit subjective probability distributions over future profit margins and unit cost growth. While the profit margin and unit cost questions yield interesting data, they are hard to formulate in a uniform manner that works well across all industries. Their inclusion also makes it harder to meet our completion-time targets, so we ultimately dropped them from our core instrument. See Appendix B for a more detailed discussion of SBU survey development and testing. The last major change to the core SBU questions occurred in September 2016.

The SBU sample covers all 50 states, all major nonfarm industries, and a range of employer size categories, as documented in Appendix A. Relative to the industry distribution of nonfarm private employment, the SBU sample materially over represents Durable Manufacturing and Finance \& Insurance. It under represents Health Care \& Social Assistance and Leisure \& Hospitality. The employment share of small firms in the SBU sample is much lower than in the U.S. private sector, especially for firms with fewer than 20 employees. The SBU covers very few firms less than five years old for three practical reasons: lags in the identification of new firms by Dunn \& Bradstreet, our infrequent purchase of business lists for cost reasons, and lags in our sampling from the lists we purchase.

All SBU data are subject to automatic review and cleaning algorithms, with further manual review of extreme outliers. Firms with more than 1,000 employees undergo manual reviews as a matter of routine. Extreme outliers and potentially anomalous responses of large firms are evaluated for consistency with historical responses and publicly available information. When 
manual reviews are inconclusive, we may contact the respondent for clarification. See Appendix A for more information and Altig et al. (2019a) for a full discussion.

\section{Measuring Subjective Expectations, Uncertainty, and Forecast Errors}

This section explains how we use the raw SBU data to compute firm-level forecasts expectations, the subjective uncertainty around the forecasts, realized outcomes, and forecast errors. For the sake of concreteness, we focus on sales growth rates in describing the measurement mechanics. The mechanics differ somewhat for investment rates, as we discuss.

Each respondent supplies future sales growth rate values, FSaleGr $r_{i}$, at support points $i=$ $1,2,3,4,5$, and the associated probabilities, $p_{i}$. We interpret the FSaleG $r_{i}$ values as conventional growth rates - i.e., percent changes on the initial value. As a preliminary step, we re-express

conventional growth rates as arc percentage changes using the formula, SaleGr $r_{i}=\frac{2 F S a l e G r_{i}}{\text { FSaleGr }_{i}+2}$. This growth rate measure is symmetric about zero, bounded between -2 and 2 , and equal to log changes up to a second-order Taylor series approximation. Growth rates computed in this manner aggregate exactly when combined with suitable weights, given by the simple mean of initial and (expected) terminal levels. This approach to growth rate measurement and aggregation is standard in the literature on business-level dynamics. See, for example, Davis and Haltiwanger (1999).

Given support points, SaleGr $r_{i}$, and probabilities, $p_{i}$, we compute mean expectations and subjective uncertainty as in (1) and (2). We compute the realized growth rate from $t$ to $t+j$ as

$$
\text { RSaleGr } r_{t, t+j}=\frac{\text { Sale }_{t+j}-\text { Sale }_{t}}{(1 / 2)\left(\text { Sale }_{t+j}+\text { Sale }_{t}\right)},
$$

where Sale $_{t}$ is reported sales at $t$. The error in the $q$-quarter ahead forecast error at month $t$ is

$$
\operatorname{Err}(\text { SaleGr })_{t}^{q}=R \text { SaleGr } r_{t, t+3 q}-\operatorname{Mean}(\text { SaleGr })_{t}^{q},
$$


where Mean(Sales) is defined by (1). For employment, we compute arc percentage changes from the reported and forecasted employment levels. ${ }^{10}$ When absolute forecast errors exceed one for sales or employment growth rates, we manually review the underlying responses and use the firm's history of responses to correct obvious mistakes such as missing or extra zeros, or the mixing of annual and quarterly sales figures. If we find no obvious mistake, we flag the observation as a likely response error and exclude it from our analysis of forecast errors.

In September and October 2017 and again in February and March 2019, we asked firms to report the book value of their capital stock (property, plant, and equipment). Starting in May 2019, we query firms every few months about book value capital stock. When available, we use the book-value capital stock as the denominator in the investment rate, I/K. When unavailable, we interpolate or extrapolate the capital stock based on the firm's reported values in other periods. If that, too, is unavailable, we use a regression-based imputation. The numerator values in the $\mathrm{I} / \mathrm{K}$ ratio come directly from our core question about capital expenditures.

Table 1 reports descriptive statistics for the support points and corresponding probabilities in our SBU data. Mean outcomes vary widely across support points, ranging for example from -0.106 to 0.115 for 12 -month employment growth rates. The mean probability mass assigned to the middle support point is about 40 percent for each outcome variable, with a mean mass of about 10 percent in each tail. Standard deviations are sizable for both support point and probability values. Table 2 reports summary statistics for forecast means, subjective uncertainty, and realized outcomes. The data exhibit considerable heterogeneity across firms in terms of realized outcomes,

\footnotetext{
${ }^{10}$ For sales growth rates (and investment rates), we work with observations that are $j=4$ quarters apart. For employment growth rates, we work with observations that are $j$ months apart, where $j$ ranges from 10 to 14 . When $j \neq 12$, we re-state the employment growth rate in annualized terms.
} 
forecast means, and subjective uncertainty. This heterogeneity is useful for analysis and reassuring in light of much previous work on the heterogeneity of realized firm-level outcomes.

\section{Properties of Subjective Distributions, Uncertainty, and Forecast Errors}

We now document several properties of the firm-level subjective forecast distributions. Our analysis sample covers SBU survey waves from October 2014 to October 2019. For the sake of brevity, we focus on results for sales growth rates. Results are very similar and often sharper for employment growth rates, as shown in Appendix C. Many qualitatively similar patterns hold for investment rates as well.

\section{Expected Growth Rates Predict Realized Growth Rates}

Figure 2 provides evidence that firm-level sales growth rate forecasts have predictive power for realized growth rates at a four-quarter look-ahead horizon. Panel (a) displays a bin scatter of firm-level values for $R$ SaleGr $r_{t, t+3 q}$ in (3) against the corresponding four-quarter ahead expected growth rates at $t$, given by Mean (SaleGr $)_{t}^{q}$ in (1). It also reports the corresponding OLS regression run on the underlying firm-level data. The estimated slope coefficient on the expected growth rate is 0.59 with a firm-clustered standard error of 0.08 , soundly rejecting the null of a unit slope coefficient. We are mindful, however, that measurement errors in the firm-level expected growth rates are likely to impart a downward bias in the OLS slope coefficient. ${ }^{11}$ Indeed, using the value of the middle support point to instrument for the firm's expected sales growth rate yields a coefficient of 0.87 (0.13), insignificantly different from one. Alternatively, using the contemporaneous expected employment growth rate as an instrument yields a coefficient of 1.12

\footnotetext{
${ }^{11}$ Measurement errors can arise because the respondent's (mean) forecast is truly noisy, because our question design elicits a noisy representation of the respondent's true forecast distribution, or for more mundane reasons - e.g., a respondent who mistypes when entering support point values or probabilities.
} 
(0.49). In short, IV regressions provide little evidence against the hypothesis of unbiased expectations. These results are consistent with Barrero (2020), who also finds little evidence of unconditional bias in firm-level expected growth rates using SBU data.

Firm-level expected sales growth rates continue to have predictive power for realized sales growth rates when we add controls for time and firm fixed effects, as shown in Panels (b) and (c) of Figure 2. The sample average of a firm's expected sales growth rate values also has strong predictive content for the average of its realized sales growth rates, as shown in Panel (d).

\section{$\underline{\text { Subjective Uncertainty Predicts the Magnitude of Forecast Errors }}$}

Figure 3 provides evidence that subjective uncertainty, as measured by (2), is highly predictive of the absolute value of the forecast errors in (4). Panel (a) shows a strong, positive relationship in the raw data. Including time fixed effects has little impact on the fitted relationship, as seen in Panel (b). Including firm effects as well weakens the relationship, but still yields a positive, significant relationship of error magnitudes to uncertainty. In other words, changes in firm-level subjective uncertainty are predictive of changes in the magnitude of firm-level forecast errors. We conclude that our measure of subjective uncertainty captures more than persistent crossfirm differences in uncertainty. The cross-firm relationship of absolute forecast errors to subjective uncertainty is indeed a strong and prominent feature of the SBU data, as shown in Panel (d). Subjective uncertainty also falls with firm size and age, as shown in Appendix C. These patterns are reassuring, given that growth rate dispersion and volatility fall with firm size and age. See, for example, Davis and Haltiwanger (1992), Caves (1998), and Davis et al. (2006).

While there is strong evidence that subjective uncertainty predicts the magnitude of forecast errors, it does not follow that firms accurately perceive the (expected) magnitude of their errors. Barrero (2020) examines this issue using SBU data. To do so, he samples from the 
subjective forecast distributions to generate the implied distribution of forecast errors. This implied distribution is much narrower than the actual distribution of forecast errors: The average magnitude of actual forecast errors is four times larger than the average magnitude of implied errors. Barrero also shows that over confidence about forecast precision holds for large and small firms but tends to fall with firm size. Ben-David et al. (2013) find that CEOs are over confident about the precision of their forecasts of returns on the S\&P 500. Taken together, these studies say that senior managers appear overconfident about their forecast precision with respect to developments at their own firms and in the broader economy.

$\underline{\text { Respondents Update Reported Beliefs Often, Usually by Small Amounts }}$

We now investigate how individual respondents update their forecast distributions over time. ${ }^{12}$ Table 3 shows that nearly all respondents provide a different forecast distribution in month $t$ for each outcome than they provided 2 or 3 months earlier in their previous survey response. For example, 95.7 percent of sales growth responses involve different support points between nearest surveys, and 94.7 percent involve different probabilities. Over 99 percent of the sales responses in consecutive surveys imply revisions to the first and second moments of the subjective probability distributions. Clearly, respondents do not supply a "boilerplate" distribution each month without thinking. Instead, they nearly always modify their reported subjective probability distributions.

To get a handle on how much they revise reported beliefs, we compute the cosine similarity of their support point and probability vectors between nearest same-topic surveys. For any pair of vectors $x$ and $x^{\prime}$ in $\mathbb{R}^{n}$, cosine similarity is the cosine of the angle between them:

\footnotetext{
${ }^{12}$ Reputational concerns and attention-seeking behavior can lead professional forecasters to distort their reported beliefs in ways that yield herding or extreme forecasts. See, for example, Lamont (2002) and Marinovic et al. (2013). Because SBU respondents are anonymous and forecast own-firm outcomes, there is no reason to anticipate such behavior in our setting. Thus, we focus on the frequency, magnitude, and character of forecast revisions.
} 


$$
\cos (\theta)=\frac{x^{\prime} \cdot x}{\left\|x^{\prime}\right\|\|x\|}
$$

where "." denotes the inner product and $\|x\|$ is the Euclidean norm of $x$. Cosine similarity ranges from -1 to 1 . Two vectors pointing in exactly the same direction (for which $\theta=0$ ) have $\cos (\theta)=$ 1 , orthogonal vectors have cosine $=0$, and vectors pointing in exactly opposite directions have cosine $=-1$. Accordingly, higher cosine similarity means that two vectors are more similar in the geometric sense of pointing in more similar directions.

Table 4 reports the mean of cosine similarity values across surveys 2 or 3 months apart for the same firm. The mean cosine values are mostly between 0.84 and 0.89 and significantly different from 1. The cosine similarity of support points for future investment is larger at 0.976 , yet still significantly different from one. Thus, we find clear evidence that respondents update their forecast distributions between surveys, while on average maintaining broadly similar responses (since the mean similarity is much closer to 1 than 0 ). For future investment, respondent support points are much more similar across nearest surveys. This result suggests that anticipated investment is revised less often than sales and employment growth expectations.

Table 5 quantifies the persistence of reported beliefs by fitting AR(1) models to subjective expectations and uncertainty of firm-level sales growth rates. The raw panel regressions in columns (1), (4), and (7) and the specifications with time fixed effects in (2), (5), and (8) show autocorrelations of just over 0.6 for subjective expectations, somewhat above 0.75 for subjective uncertainty, and about 0.66 for log-subjective uncertainty. These results suggest that shocks to firm-level sales growth rate expectations decay by about forty percent between nearest surveys and by about one-fourth to one-third for subjective uncertainty (in levels versus $\operatorname{logs}$ ). Autocorrelations are smaller at about 0.25 or less when we also condition on firm effects, but still positive and statistically significant. 


\section{$\underline{\text { Subjective Uncertainty Predicts the Magnitude of Future Forecast Revisions }}$}

Having established that SBU respondents actively revise their reported beliefs, we next consider how subjective uncertainty relates to the magnitude of the firm's future forecast revisions. Figure 4 shows that firms reporting greater uncertainty today make larger future revisions to their expectations. The figure plots the absolute change in the sales growth rate expectation from $t$ to $t+2$ (or $t+3$ ) against subjective uncertainty about the sales growth rate at $t$. The positive relationship between a firm's subjective uncertainty today and the magnitude of its future expectation revision holds in the raw data and when controlling for firm and time fixed effects.

Figure 5 shows that a firm's current subjective uncertainty is also predictive of future revisions to its subjective uncertainty. The figure plots the absolute change in a firm's sales growth rate uncertainty from $t$ to $t+2$ (or $t+3$ ) against subjective uncertainty about its sales growth rate at t. Again, the positive relationship holds in the raw data and when controlling for firm and time fixed effects. Perhaps surprisingly, firm-level uncertainty at $t$ has even more predictive power for the magnitude of future revisions to the firm's uncertainty than for the magnitude of future revisions to its expectation.

\section{Do Revisions in Expectations Predict Future Forecast Errors?}

We now consider whether revisions in a firm's expectations predict its future forecast errors, following Coibion and Gorodnichenko (2015). In particular, we regress the error in the sales growth rate forecast formed at $t$ in equation (4) on a constant and same-firm changes in Mean $(\text { SaleGr })_{t-j}^{q}-$ Mean $(\text { SaleGr })_{t}^{q}$, for $j=2$ or 3. The regression, which has 2,177 firm-level observations, yields a slope coefficient of $0.335(0.09)$ and an R-squared value of 0.008 . The positive slope suggests that business executives over extrapolate from recent news in forming 
expectations about the future growth of their firms. They are both too optimistic in the wake of good news, and too pessimistic in the wake of bad news.

Measurement error in the reported expectations could also drive the positive slope coefficient in this regression. In this regard, note that Mean $(\text { SaleGr })_{t}^{q}$ enters both the dependent and independent variables in the regression. Partly motivated by concerns about measurement error, Barrero (2020) presents a broader range of tests and concludes that over extrapolation is a feature of managerial beliefs in SBU data. Gennaioli, Ma and Shleifer (2016) present evidence that points to over extrapolation in the expectations reported in the Duke Survey of CFOs.

Subjective Uncertainty Has a V-shaped Relation to Past Growth and Forecast Revisions

Subjective uncertainty is associated with larger revisions in future beliefs (Figures 4 and 5), but what drives subjective uncertainty? To throw light on this matter, Figure 6 displays two bin scatters with $S D(\text { SaleGr })_{t}^{q}$, uncertainty about the $q$-quarter ahead forecast at $t$, on the vertical axis. Panel (a) relates this measure of subjective uncertainty to $R S a l e G r_{t-12, t}$, the realized sales growth rate over the previous year. Firms with greater absolute growth rates in the past year report higher subjective uncertainty, yielding a pronounced V-shaped pattern. This result is consistent with models in which firms have stochastic volatility - they go through periods of higher and lower volatility. Large recent shocks in these models are associated with higher levels of current volatility, and hence higher future uncertainty.

We can assess this stochastic volatility interpretation directly by exploiting the panel structure of the SBU. To do so, panel (b) in Figure 6 relates subjective uncertainty at $t$ to the 
absolute value of the firm's most recent expectation revision given by $\mid$ Mean $(\text { SaleGr })_{t-j}^{q}-$ $\operatorname{Mean}(\text { SaleGr })_{t}^{q} \mid$, for $j=2$ or 3 . Again, we see a pronounced V shape. ${ }^{13}$

Next, we nest these two effects in a single regression model. Specifically, we regress subjective uncertainty at $t$ on a constant, the firm's absolute growth rate over the previous year, and the absolute value of its most recent expectation revision. This regression, which has 4,722 observations, yields a coefficient of $0.119(0.012)$ on $\left|R S a l e G r_{t-12, t}\right|$ and a coefficient of 0.228 (.025) on $\mid$ Mean (SaleGr $)_{t-j}^{q}-$ Mean(SaleGr $)_{t}^{q} \mid$, for $j=2$ or 3 . The R-squared value is 0.362 . That is, subjective uncertainty rises with the firm's absolute growth rate in the recent past and with the magnitude of its recent forecast revisions. Using employment counterparts to the sales measures yields very similar regression results, with an R-squared value of 0.336 . These results say that subjective uncertainty is high in the wake of large recent changes to the firm's activity level and in the wake of large revisions to its future growth prospects. Both effects are present in the data, and neither drives out the other in our regression specifications.

\section{Indices of Business Expectations and Uncertainty for the US Economy}

This section describes how we use SBU data to construct indices of business expectations and uncertainty. Our basic approach is to compute size-weighted averages of first and second moments in the firm-level subjective forecast distributions. As before, the look-ahead horizon is twelve months for employment growth and four quarters for sales growth and investment.

In constructing the indices, we winsorize firm-level mean forecasts and subjective uncertainty values at the $1^{\text {st }}$ and $99^{\text {th }}$ percentiles in the fixed period from January 2015 to December

\footnotetext{
${ }^{13}$ In unreported results, subjective uncertainty at $t$ also has a V-shaped relation to the error in the sales growth rate expectation formed one year-earlier. This relationship is noisier than the ones shown in Figure 6 and is derived from a smaller sample.
} 
2018. In averaging the winsorized values over firms, we weight by the firm's employment level, top coded at 500. The top coding of activity weights reflects our judgment based on long experience in analyzing business-level data. Outliers and errors for large firms can seriously distort sample-average quantities, more so for samples of modest size and for higher-order moments. ${ }^{14}$

Figure 7 displays smoothed expectation and uncertainty indices for sales and employment growth rates. ${ }^{15}$ We have about 50 firm-level responses per topic per month before September 2016 and 150 thereafter. To generate index values that reflect similar observation counts per month, we smooth as follows: From November 2016 onwards, we use a three-month lagged moving average; in August 2016 and earlier, we use a nine-month lagged moving average; and for September and October 2016, we use a seven-month and five-month lagged moving average, respectively.

The first-moment indices in the left panel of Figure 7 show expected one-year growth rates of less than 1 percent in 2015 and most of 2016 for employment and less than 2 percent for sales. Expected growth rises through early 2018 for employment and through late 2018 for sales, reaching 5 percent near the end of 2018. Growth rate expectations fall thereafter but stay above 2 percent for employment and near 4 percent for sales by October 2019. The second-moment indices in the right panel show falling subjective uncertainty about future growth rates through the middle of 2017, except for an upswing in sales growth uncertainty around the November 2016 election.

\footnotetext{
${ }^{14}$ Our raw sales indices show a marked level and volatility break in September 2016, when we revised the formulation of our sales questions. To adjust for this break, we first compute the time-series mean and standard deviation of the employment expectation index values in the "pre" period (before September 2016) and the "post" period (September 2016 to December 2018). Second, we compute the pre-to-post ratio of means and the pre-to-post ratio of standard deviations for employment expectations. Last, we adjust the pre-period sales expectation index values, so that the pre-to-post ratios of means and standard deviations for the sales expectation index match the corresponding ratios for the employment expectations index. We take the same approach in adjusting the subjective uncertainty index values for sales growth. ${ }^{15}$ The corresponding indices for $\mathrm{I} / \mathrm{K}$, shown in Appendix D, are noisier. Greater noisiness in the $\mathrm{I} / \mathrm{K}$ series could reflect the lumpiness of firm-level investment (especially with our modest sample size), our heavy use of imputed values for firm-level capital stocks, or weaknesses in our question design for capital expenditures. Evaluating and improving our forward-looking $\mathrm{I} / \mathrm{K}$ measures is on our agenda.
} 
Since early 2017, the cross-firm average subjective uncertainty measures have settled into narrow ranges near 3 percent for sales and 4 percent for employment.

The recent stability of our subjective uncertainty measures may seem surprising in light of the extraordinary rise in trade policy uncertainty since March 2018. (Baker, Bloom and Davis (2019) review several pieces of evidence). As part of our special question series, we asked SBU panel members about trade policy developments in January, July, August and September of 2019. The resulting data show mounting concerns about tariff hikes and trade policy tensions and evidence of their negative effects on employment, sales and investment. The effects are modest in size, however, and a majority of SBU panel members report little direct exposure to trade policy developments (Altig et al., 2019bc). Taken together, Figure 7 and our earlier reports suggest two conclusions. First, other sources of business-level uncertainty diminished after early 2018, muting or offsetting the impact of rising trade policy uncertainty. Second, trade policy developments contributed to the falling growth rate expectations in 2018 and 2019.

To our knowledge, there are no alternative time-series measures for the United States that quantify the same concepts as our SBU indices. So, we turn to some admittedly imperfect comparisons. The Duke University Survey of CFOs at U.S. firms includes the following question: “Relative to the previous 12 months, what will be your company's PERCENTAGE CHANGE [in revenues] during the next 12 months. (e.g., $+3 \%, 0 \%,-2 \%$, etc.)?" That is, the Duke survey elicits the expected change in growth rates from the past year to the year ahead. In contrast, the SBU yields the expected growth rate in the year ahead. Nevertheless, one might expect the two surveys to yield positively correlated first-moment indices. That turns out to be the case, as seen in the left panel of Figure 8. We plot the revenue-weighted mean in month $t$ of firm-level responses to the 
Duke survey question above alongside our SBU sales growth rate expectations index at $t$. The two series exhibit broadly similar movements over our sample period.

The right panel in Figure 8 shows our sales growth uncertainty index alongside a smoothed version of the one-year-ahead VIX, which measures the volatility of the S\&P 500 implied by options set to expire one year hence. ${ }^{16} \mathrm{We}$ focus on the VIX because it is well known, widely used, and often seen as a proxy for broad economic uncertainty. In fact, the VIX is better understood as measuring the expected magnitude over the option horizon of news about the stock market value of larger, listed firms. ${ }^{17}$ Despite the conceptual differences, Figure 8 reveals that our uncertainty index correlates positively with the VIX, especially since late 2016.

The comparisons in Figure 8 suggest that our SBU indices respond to economic developments in a manner that is broadly similar to other model-free indicators of expected growth rates and economic uncertainty, namely the Duke CFO Survey and the VIX. Like the SBU, these other sources are available in (near) real time and, in the case of the Duke Survey, pertain to forecasts of own-firm outcomes.

In closing this section, we wish to stress the preliminary nature of our SBU indices. The current SBU sample is modest in size and excludes younger firms. We continue to expand the sample and refine our data auditing and cleaning methods. Like any startup survey, we need many years (or large in-sample moves) before we can confidently assess the predictive value of the

\footnotetext{
${ }^{16}$ Since the SBU is in the field during the second and third week of the month, we take the value of the one-year VIX on the $15^{\text {th }}$ of the month. If the $15^{\text {th }}$ is not a trading day, we use the $16^{\text {th }}, 14^{\text {th }}, 17^{\text {th }}, 13^{\text {th }}, 18^{\text {th }}$, or $12^{\text {th }}$ in that order. We smooth the resulting monthly one-year VIX series using the same procedure as for our SBU indices.

${ }^{17}$ These firms account for about a quarter of private sector employment, and they differ systematically from the economy as a whole on several dimensions. In particular, listed firms skew toward bigger, older, capital-intensive, skill-intensive and multinational firms. See Davis (2017). Changes in the mix of listed firms and their leverage choices also affect the VIX.
} 
aggregate SBU indices. Nevertheless, the predictive value of our firm-level subjective forecast distributions, as documented in Section 3, provides grounds for optimism in this regard.

\section{Additional Results and Robustness Checks}

The Shape of SBU Forecast Distributions Has Predictive Value

Section 3 shows that first and second moments of SBU forecast distributions have predictive value for realized future growth rates (Figure 2), the magnitude of future forecast errors (Figure 3), and the extent of future forecast revisions (Figures 4 and 5). We now investigate whether other aspects of SBU forecast distributions - beyond first and second moments - have predictive value for firm-level outcomes. ${ }^{18}$

We first ask how skewness in subjective forecast distributions relates to skewness in realized outcomes over the forecast horizon. To do so, we sort the firm-level observations into quartiles defined by the Fisher-Pearson skewness coefficients of the subjective forecast distributions. For each quartile, we compute the mean value of the subjective skewness coefficients and the skewness coefficient of realized growth rates over the forecast horizon. Figure 9 displays a scatter plot of these two measures. For employment growth rates, skewness in realized outcomes rises strongly with prior subjective skewness. For sales growth rates, the relationship is similar except for the anomalous second quartile. Overall, Figure 9 suggests that skewness in the subjective forecast distribution portends skewness in the distribution of realized outcomes.

In a second exercise, we ask whether the shape of the subjective forecast distribution has predictive value for the sign of forecast errors in the firm-level data. To do so, we regress the sign

\footnotetext{
18 The skewness of cross-sectional outcomes is known to covary in interesting ways with aggregate outcomes. See, for example, Guvenen et al. (2014) on cyclicality in the skewness of individual-level earnings shocks and Salgado et al. (2019) on the cyclicality of skewness in firm-level growth rates.
} 
of the forecast error in (4) on a constant and the fraction of mass in the subjective forecast distribution on support points greater than Mean $(\text { SaleGr })_{t}^{q}$. We test whether the coefficient on this fraction is positive and statistically significant. To see the logic, suppose the subjective and true distributions are the same. Then the probability of a positive forecast error, as defined in (4), rises with the mass on support point values greater than Mean $(\text { SaleGr })_{t}^{q}$.

Running this regression on 3,037 firm-level observations yields an estimated slope coefficient of 0.034 , with a firm-clustered standard error of 0.051. Adding time effects to control for common components in the forecast errors yields a slightly larger slope coefficient, but one that remains statistically insignificant. Thus, the shape of the subjective forecast distribution over sales growth rates has little predictive content for the sign of forecast errors. However, when we repeat the test for employment growth rates, we find strong evidence that the shape of the subjective forecast distribution predicts the sign of forecast errors. The regression, which has 3,692 firm-level observations, yields an estimated slope coefficient of 0.089 with a firm-clustered standard error of 0.046. Including time effects yields a slightly larger slope coefficient.

Finally, Appendix $\mathrm{C}$ shows that the third moment of SBU forecast distributions has predictive value for realized growth rates and the absolute value of forecast errors when conditioning on the first two subjective moments. This pattern holds for both sales and employment growth rates and is especially strong for sales. While interesting as more evidence that the shape of SBU forecast distributions has predictive value for firm-level outcomes, the interpretation is unclear. If the subjective and true forecast distributions were identical, higher moments of the subjective distribution would have no predictive power for realized growth rates after conditioning on the subjective first moment. Thus, the marginal predictive value of subjective 
skewness for realized growth rates is evidence about the nature of systematic differences between subjective and true forecast distributions.

In summary, we find strong evidence across various exercises of predictive content in the shape of SBU forecast distributions over employment growth rates. The evidence of predictive content in the shape of SBU forecast distributions over sales growth rates is weaker. We leave it for future research to explain why.

Do Repeat Applications of the Survey Instrument Affect Responses?

Repeated application of a survey instrument can influence how a respondent thinks about the survey questions, affecting his or her responses over time. Binder (2019), for example, finds that inflation forecasts and inflation uncertainty decline with the number of previous responses among participants of the New York Fed's Survey of Consumer Expectations. Patterns like these raise questions about how to interpret the survey data and their properties.

To investigate this matter in the SBU, we regress the natural logarithm of subjective uncertainty on the respondent's number of previous survey completions as of month $t .{ }^{19}$ We control for time effects, because the average number of completions among respondents at $t$ covaries with calendar time. We include firm effects to isolate within-firm variation. The results, reported in Table 6, reveal no statistically significant evidence of survey application effects. Moreover, the point estimates imply tiny effects. For example, the coefficient in column (2) says ten previous survey completions lowers the log of subjective uncertainty by -0.03 . This effect is about 1 percent of the dependent variable mean value and 3 percent of its standard deviation. Unreported results for mean expectations also reveal no evidence of survey application effects.

\footnotetext{
${ }^{19}$ Logging yields a more normally distributed outcome variable, but similar results hold when using unlogged subjective uncertainty as the dependent variable.
} 
Figure 10 reports results for a nonparametric specification that allows an unrestricted relationship between the firm's reported value of log subjective uncertainty and its number of previous completions. As before, we include firm and time fixed effects in the specification. As seen in the left panel of Figure 10, there is weak evidence of small negative survey application effects when we do not activity weight the firm-level observations. The effect appears to settle in over about nine completions and then stabilize at a value about 5-6 percent as large as the mean of the dependent variable and 20-25 percent of its standard deviation. The activity-weighted results in the right panel of Figure 10 show no indication of survey application effects. ${ }^{20}$

In summary, Table 6 and Figure 10 support three inferences. First, there is little evidence against the null that repeated survey applications have zero effect on survey responses. Second, the point estimates imply tiny survey application effects. Third, large survey application effects are quite unlikely, given the precision of the point estimates. We conclude that survey application effects on reported responses are not a major concern in the SBU.

The Impact of Replacing Discrete with Continuous Distributions

To this point, we have interpreted survey responses literally in calculating subjective moments. As remarked in Section 1.A, we don't know how respondents conceptualize uncertainty. Instead of a mass point at the "worst" case in a five-point distribution, for example, the respondent might contemplate a range of bad outcomes. Rather than a discrete distribution, respondents might think in terms of continuous or mixed distributions. To get some sense of whether this issue matters much, we now interpret responses as approximations to an underlying continuous distribution.

Let $g_{i}$ and $p_{i}$ denote support points and probabilities in the raw survey data for $i=$ $1,2,3,4,5$. Assume that these survey responses derive from the following continuous density:

\footnotetext{
${ }^{20}$ This pattern suggests that repeated application of the survey instrument has modest negative effects on the subjective uncertainty reported by small firms.
} 


$$
f(x)=\left\{\begin{array}{cl}
\frac{p_{1}}{g_{2}-g_{1}}, & \text { for } x \in\left[\frac{3 g_{1}-g_{2}}{2}, \frac{g_{1}+g_{2}}{2}\right), \\
\frac{p_{i}}{\left(g_{i+1}-g_{i-1}\right) / 2}, & \text { for } x \in\left[\frac{g_{i-1}+g_{i}}{2}, \frac{g_{i}+g_{i+1}}{2}\right), \\
\frac{p_{5}}{g_{5}-g_{4}}, & \text { for } x \in\left[\frac{g_{4}+g_{5}}{2}, \frac{3 g_{5}-g_{4}}{2}\right] .
\end{array}\right.
$$

Equation (5) specifies five adjoining uniform density segments. The leftmost segment is centered at $g_{1}$, the "worst" forecast outcome in the raw survey data. It extends leftward from $g_{1}$ by $\left(g_{1}-g_{2}\right) / 2$ units and rightward by $\left(g_{2}-g_{1}\right) / 2$. Given its length, the height of the density segments is selected to exhaust, $p_{1}$, the mass assigned to the "worst" outcome in the raw data. The next segment extends from $\left(g_{1}+g_{2}\right) / 2$ to $\left(g_{2}+g_{3}\right) / 2$ and so on, with the height of each density segment selected to exhaust the corresponding mass point in the raw data. In other words, equation (5) takes the mass assigned to each support point in the raw data and spreads it uniformly in symmetric interval around the support point.

Figure 11 compares the first and second moments generated from (5) to the corresponding moments computed directly from the SBU data. The two approaches to moment calculation yield nearly identical results over almost the entire range of sales growth rates in the data. Only for the $1^{\text {st }}$ and $99^{\text {th }}$ quantiles of $\log$ subjective uncertainty do we see notable deviations between the discrete and continuous interpretations of the data. In Appendix C, we also show that continuous and discrete interpretations of SBU data perform equally well with respect to the predictive value of mean expectations for realized growth rate outcomes. The discrete interpretation performs slightly better with respective to the predictive value of subjective uncertainty for the magnitude of absolute forecast errors. These results suggest that the five-point probability distributions elicited by the SBU are not an important source of approximation errors. 
How Do Sample Composition Changes Affect Our Expectation and Uncertainty Indices?

The SBU is a panel survey with entry and attrition over time. That raises the possibility that sample mix changes could materially impact movements in the first- and second-moment indices in Section 4. To explore this matter, we fit employment-weighted regressions of the form,

$$
y_{f t}=\alpha_{f}+\beta_{t}+\varepsilon_{f t},
$$

where $y_{f t}$ is a measure of growth rate expectations or subjective uncertainty for firm $f$ in month $t, \alpha_{f}$ is a vector of firm fixed effects, and $\beta_{t}$ is a vector of time effects. The estimated $\beta_{t}$ constitute a time series of employment-weighted outcomes that control for changes in the mix of firms in the sample. Dropping $\alpha_{f}$ in (6) and refitting an employment-weighted regression, the estimated $\beta_{t}$ recover the original indices described in Section 4.

Figure 12 displays the results of fitting (6) - with and without firm fixed effects - for sales growth rate expectations and subjective uncertainty about sales growth rates. Controlling for sample composition has a sizable impact on the evolution of the expectations and uncertainty indices until late 2016 but matters little thereafter. In this regard, we note that our sample has become larger and more representative of the U.S. industry distribution over time. These sample improvements explain why sample composition effects have diminished over time.

\section{Reweighting to Match the Industry and Regional Distribution of Activity}

Another set of issues relates to sample representativeness. An unrepresentative sample may yield biased estimates of population moments. Moreover, because the SBU oversamples cyclically sensitive industries, common shocks may generate larger responses of the (activity-weighted) average subjective forecast distributions in the sample than in the economy. To investigate these issues, we reweight the firm-level observations in the SBU sample to target the distribution of activity by industry and region in the U.S. economy. We then use the reweighted data to construct 
alternative indices. Appendix D explains how we reweight, presents the alternative indices, and compares them to the baseline indices in Figures 7 and 8.

For employment growth rates, reweighting raises the average expectations value and lowers the average uncertainty value in 2015 and most of 2016. These gaps nearly vanish by late 2016: From September 2016 onwards, the alternative and baseline indices differ by only 10 basis points for the average expectation of employment growth rates and by only 8 basis points for the corresponding average subjective uncertainty. A similar pattern holds for the sales growth rate indices, but modest gaps persist: From September 2016 onwards, reweighting lowers the average expectation (subjective uncertainty) of sales growth rates by about 35 (27) basis points. Happily, reweighting has little impact on the cyclical behavior of the sales growth rate uncertainty index. The same is true for the sales growth rate expectations index after September 2016. The alternative indices are often noisier than the baseline indices, especially before September 2016. This pattern is a consequence of upweighting firm-level observations in thin cells. Recall that we have only about 50 observations per month per outcome variable before September 2016. To sum up, the representativeness of the SBU sample appears adequate for drawing reliable inferences about business expectations and uncertainty in the U.S. economy since at least September 2016.

\section{Concluding Remarks}

We develop and field a new panel survey of business executives that elicits subjective forecast distributions over own-firm future outcomes. In terms of question design, our key innovation lets survey respondents freely select support points and probabilities in five-point distributions. In terms of data collection, our monthly panel Survey of Business Uncertainty covers about 1,750 firms drawn from all 50 states, every major nonfarm industry, and a range of firm 
sizes. We continue our efforts to expand the panel, improve the quality of SBU data, and better understand how business managers conceptualize uncertainty and form forecasts.

SBU respondents update their forecast distributions frequently, usually by small amounts. When respondents express greater uncertainty today, they make larger future revisions to their forecast distributions. These patterns suggest that respondents are attentive to the survey, and that they supply meaningful data. Indeed, we show that the subjective forecast distributions have predictive power for firm-level sales and employment growth rates in multiple respects: Subjective expectations are predictive of realized growth rates. Subjective uncertainty (the standard deviation of the forecast distribution) is predictive for the magnitude of future forecast errors and the extent of future forecast revisions.

We also develop evidence about the conditions that lead to high subjective uncertainty over own-firm future outcomes. Specifically, subjective uncertainty has a pronounced V-shaped relation to the firm's recent past growth rate and to the firm's most recent revision to its expected growth rate. In other words, large recent changes and large recent forecast revisions lead to high forward-looking uncertainty. As the sample grows and firm-level response histories lengthen, the SBU will become increasingly useful for analyzing the determinants of subjective uncertainty and other aspects of belief formation and revision.

Finally, we use the SBU micro data to build monthly indices of aggregate U.S. business expectations and uncertainty for sales growth rates, employment growth rates, and investment rates at a one-year look-ahead horizon. We began publishing these indices in November 2018, and they are now carried by Bloomberg, FRED, and Haver Analytics. We regard these indices as works in progress, but we hope they will aid policymakers and analysts in assessing the outlook for the US economy and the extent of uncertainty about the outlook. 


\section{References}

Altig, David, Jose Maria Barrero, Nick Bloom, Mike Bryan, Steven J. Davis, Brent Meyer, and Nick Parker, 2019a. "The Survey of Business Uncertainty: Data Audit and Edit Methodology," Technical Report, Federal Reserve Bank of Atlanta.

Altig, David, Nick Bloom, Steven J. Davis, Brent Meyer and Nick Parker, 2019b. "Tariff Worries and U.S. Business Investment, Take Two," Macroblog, Federal Reserve Bank of Atlanta, 25 February.

Altig, David, Jose Maria Barrero, Nick Bloom, Steven J. Davis, Brent Meyer, and Nick Parker, 2019c. "New Evidence Points to Mounting Trade Policy Effects on U.S. Business Activity," Macroblog, Federal Reserve Bank of Atlanta, 1 November.

Akepanidtaworn, Klakow, Rick Di Mascio, Alex Imas and Lawrence Schmidt, 2019. "Selling Fast and Buying Slow: Heuristics and Trading Performance of Institutional Investors," working paper.

Armantier, Olivier, Giorgio Topa, Wilber van der Klaauw and Basit Zafar, 2017. "An Overview of the Survey of Consumer Expectations," Economic Policy Review, 23, no. 2.

Awano, Gaganan, Nicholas Bloom, Ted Dolgy, Paul Mizen, Rebecca Riley, Tatsuro Senga, John Van Reenen, Jenny Vyas and Philip Wales, 2018. "A Firm-Level Perspective on Microand Macro-level Uncertainty," ESCoE Discussion Paper 2018-10, July.

Bachmann, Ruediger, Kai Carstensen, Stefan Lautenbacher and Martin Schneider, 2018. "Uncertainty and Change: Survey Evidence of Firms' Subjective Beliefs," working paper.

Bachmann, Ruediger and Stefan Elstner, 2015. "Firm optimism and pessimism," European Economic Review, 79 (October), 297-325.

Bachmann, Ruediger, Stefan Elstner and Eric R. Sims, 2013. "Uncertainty and economic activity: Evidence from business survey data," American Economic Journal: Macroeconomics, 5, no. 2, 217-49.

Bailey, Warren, Alok Kumar and David Ng, 2011. "Behavioral biases of mutual fund investors," Journal of Financial Economics, 102, no. 1, 1-27.

Baker, Scott R., Nicholas Bloom and Steven J. Davis, 2016. "Measuring economic policy uncertainty," Quarterly Journal of Economics, 131, no. 4, 1593-1636.

Baker, Scott R., Nicholas Bloom and Steven J. Davis, 2019. "The Extraordinary Rise in Trade Policy Uncertainty," VOX CEPR Policy Portal, 17 September.

Barrero, Jose Maria, 2020. "The Micro and Macro of Managerial Beliefs," working paper, Instituto Tecnológico Autónomo de México.

Barber, Brad and Terrance Odean, 2001. "Boys will be boys: Gender, overconfidence, and common stock investment," Quarterly Journal of Economics, 116, no. 1, 261-292.

Ben-David, Itzhak, John R. Graham, J. R. and Campbell R. Harvey, 2013. "Managerial Miscalibration," Quarterly Journal of Economics, 128, no. 4, 1547-1584. 
Binder, Carola C., 2019. "Panel Conditioning in the Survey of Consumer Expectations," working paper.

Bloom, Nicholas, 2009. "The impact of uncertainty shocks," Econometrica, 77, no. 3, 623-685.

Bloom, Nicholas, Philip Bunn, Scarlet Chen, Paul Mizen, Pawel Smietanka, Greg Thwaites and Garry Young, 2018a. "Brexit and uncertainty: insights from the Decision Maker Panel," Fiscal Studies, 39, no. 4, 555-580.

Bloom, Nicholas, Hong Cheng, Mark Duggan, Hongbin Li and Franklin Qian, 2018b. "Do CEOs Know Best? Evidence from China," NBER Working Paper No. 24760.

Bloom, Nicholas, Steven J. Davis, Lucia Foster, Brian Lucking, Scott Ohlmacher and Itay Saporta Ecksten, 2017. "Business-Level Expectations and Uncertainty," working paper.

Bordalo, Pedro, Nicola Gennaioli, Rafael La Porta and Andrei Shleifer, 2019. "Diagnostic Expectations and Stock Returns," Journal of Finance, 74, no. 6, 2839-2874.

Caves, Richard E., 1998. "Industrial Organization and New Findings on the Turnover and Mobility of Firms," Journal of Economic Literature, 36 (December), 1947-1982.

Chen, Cheng, Tatsuro Senga, Chang Sun and Hongyong Zhang, 2019. "Uncertainty, Imperfect Information and Learning in the International Market," working paper.

Coibion, Olivier and Yuriy Gorodnichenko, 2012. "What can survey forecasts tell us about information rigidities?” Journal of Political Economy, 120, no. 1, 116-159.

Coibion, Olivier and Yuriy Gorodnichenko, 2015. "Information rigidity and the expectations formation process: A simple framework and new facts," American Economic Review, 105, no. 8, 2644-78.

Colacito, Riccardo, Mariano M. Croce, Liu, Yang Liu and Ivan Shaliastovich, 2018. "Volatility risk pass-through," National Bureau of Economic Research Working Paper No. 25276.

Davis, Steven J., 2017. "Policy Uncertainty vs. the VIX: Streets and Horizons," proceedings of the Federal Reserve Board Workshop on Global Risk, Uncertainty and Volatility, Washington, DC. At https://faculty.chicagobooth.edu/steven.davis/speaking.html.

Davis, Steven J. and John Haltiwanger, 1992. "Gross Job Creation, Gross Job Destruction, and Employment Reallocation," Quarterly Journal of Economics, 107, no. 3 (August), 819863.

Davis, Steven J. and John Haltiwanger, 1999. "Gross job flows," Handbook of Labor Economics, Volume 3, edited by Orley Ashenfelter and David Card. Elsevier Science B.V.

Davis, Steven J. and John Haltiwanger, Ron S. Jarmin and Javier Miranda, 2006. "Volatility and dispersion in business growth rates: Publicly traded versus privately held firms," NBER Macroeconomics Annual, 21, 107-179.

Dominitz, Jeff and Charles F. Manski, 1997. "Using expectations data to study subjective income expectations," Journal of the American Statistical Association, 92, no. 439, 855867.

Datta, Deepa, Juan M. Londono, Bo Sun, Daniel O. Beltran, Thiago R.T. Ferreira, Matteo M. Iacoviello, Mohammad R. Jahan-Parvar, Canlin Li, Marious Rodriguez and John H. Rogers, 2017. "Taxonomy of Global Risk, Uncertainty, and Volatility Measures," Board 
of Governors of the Federal Reserve System, International Finance Discussion Papers Number 1216.

Federal Reserve Bank of New York, 2013. Empire State Manufacturing Survey, May.

Fernández-Villaverde, Jesus, Pablo Guerron-Quintana, Juan F. Rubio-Ramírez, and Martin Uribe, 2011. "Risk matters: The real effects of volatility shocks," American Economic Review, 101, no. 6, 2530-61.

Friend, Irwin and Jean Bronfenbrenne, 1955. "Plant and Equipment Programs and Their Realization," in Short-Term Economic Forecasting, Conference on Research in Income and Wealth. Princeton University Press.

Gennaioli, Nicola, Yueran Ma and Andrei Shleifer, 2016. "Expectations and investment," NBER Macroeconomics Annual, 30, 379-431.

Guiso, Luigi and Giuseppe Parigi, 1999. "Investment and demand uncertainty," Quarterly Journal of Economics, 114, no. 1, 185-227.

Guvenen, Fatih, Serdar Ozkan and Jae Song, 2014. "The nature of countercyclical income risk," Journal of Political Economy, 122, no. 3, 621-660.

Handley, Kyle and J. Frank Li (2018). "Measuring the Effects of Firm Uncertainty on Economic Activity: New Evidence for One Million Documents," University of Michigan.

Hassan, Tarek A., Stephan Hollander, Laurence van Lent and Ahmed Tahoun, 2019. "Firm-level political risk: Measurement and effects," Quarterly Journal of Economics, 134, no. 4 (November), 2135-2202.

Hurd, Michael D., 1999. "Anchoring and Acquiescence Bias in Measuring Assets in Household Surveys," Journal of Risk and Uncertainty, 19, 111-136.

Jurado, Kyle, Sydney Ludvigson and Serena Ng, 2015. "Measuring uncertainty," American Economic Review, 105, no. 3, 1177-1216.

Juster, F. Thomas and Richard Suzman, 1995. "An Overview of the Health and Retirement Study," Journal of Human Resources, 30, S7-S56.

Kozeniauskas, Nicholas, Anna Orlik and Laura Veldkamp, 2018. "What are uncertainty shocks?” Journal of Monetary Economics, 100 (December), 1-15.

Lamont, Owen, 2002. "Macroeconomic forecasts and microeconomic forecasters," Journal of Economic Behavior and Organizations, 48, no. 3, 265-280.

Malmendier, Ulrike and Geoffrey Tate, 2005. "CEO overconfidence and corporate investment," Journal of Finance, 60, no. 6, 2661-2700.

Marinovic, Ivan, Marco Ottaviani and Peter Sorensen, 2013. "Forecasters' Objectives and Strategies," in Graham Elliot and Allan Timmerman, editors, Handbook of Economic Forecasting, Volume 2, Part B, 690-720.

Manski, Charles F. 2004. "Measuring expectations,” Econometrica, 72, no. 5, 1329-1376.

Manski, Charles F. 2018. "Survey measurement of probabilistic macroeconomic expectations: progress and promise." NBER Macroeconomics Annual, 32, 411-471. 
Massenot, Baptiste and Yuri Pettinicchi, 2018. "Can firms see into the future? Survey evidence from Germany," Journal of Economic Behavior \& Organization, 145, 66-79.

Puetz, Alexander and Stefan Ruenzi, 2011. "Overconfidence among professional investors: Evidence from mutual fund managers," Journal of Business Finance \& Accounting, 38, nos. 5-6, 684-712.

Rozsypal, Filip and Kathrin Schlafmann, 2017. "Overpersistence bias in individual income expectations and its aggregate implications," CEPR Discussion Paper No. DP12028.

Salgado, Sergio, Fatih Guvenen and Nicholas Bloom, 2019. "Skewed business cycles," National Bureau of Economic Research Working Paper no. 26565.

Scotti, Chiara, 2016. "Surprise and uncertainty indices: Real-time aggregation of real-activity macro-surprises," Journal of Monetary Economics, 82, 1-19.

Tanaka, Mari, Nicholas Bloom, Joel M. David and Maiko Koga, 2019. "Firm Performance and Macro Forecast Accuracy," Journal of Monetary Economics, forthcoming. 


\section{Table 1: Summary Statistics for Support Points and Probabilities}

\begin{tabular}{|c|c|c|c|c|c|c|}
\hline \multirow{3}{*}{ Support Point } & \multicolumn{6}{|c|}{ Support Point Outcomes } \\
\hline & \multicolumn{2}{|c|}{$\begin{array}{c}\text { Employment Growth Rate, } \\
\text { Next } 12 \text { Months }\end{array}$} & \multicolumn{2}{|c|}{$\begin{array}{l}\text { Sales Growth Rate, } \\
\text { Next } 4 \text { Quarters }\end{array}$} & \multicolumn{2}{|c|}{$\begin{array}{c}\text { Investment Rate (I/K), } \\
4 \text { Quarters Ahead }\end{array}$} \\
\hline & Mean & SD & Mean & SD & Mean & SD \\
\hline 1 & -0.106 & 0.166 & -0.042 & 0.123 & 0.047 & 0.143 \\
\hline 2 & -0.049 & 0.111 & 0.001 & 0.087 & 0.073 & 0.203 \\
\hline 3 & 0.014 & 0.080 & 0.044 & 0.075 & 0.110 & 0.293 \\
\hline 4 & 0.067 & 0.095 & 0.081 & 0.086 & 0.152 & 0.390 \\
\hline 5 & 0.115 & 0.124 & 0.121 & 0.111 & 0.213 & 0.522 \\
\hline \multirow[t]{2}{*}{$\mathrm{N}$} & \multicolumn{2}{|c|}{7064} & \multicolumn{2}{|c|}{7159} & \multicolumn{2}{|c|}{6433} \\
\hline & & & upport $P$ & ilities $(\%$ & & \\
\hline \multirow[t]{2}{*}{ Support Point } & \multicolumn{2}{|c|}{$\begin{array}{c}\text { Employment Growth Rate, } \\
\text { Next } 12 \text { Months }\end{array}$} & \multicolumn{2}{|c|}{$\begin{array}{c}\text { Sales Growth Rate, } \\
\text { Next } 4 \text { Quarters } \\
\end{array}$} & \multicolumn{2}{|c|}{$\begin{array}{l}\text { Investment Rate, } \\
4 \text { Quarters Ahead }\end{array}$} \\
\hline & Mean & SD & Mean & SD & Mean & SD \\
\hline 1 & 10.875 & 9.438 & 12.438 & 11.399 & 12.770 & 12.310 \\
\hline 2 & 18.318 & 9.621 & 18.799 & 9.346 & 18.793 & 9.200 \\
\hline 3 & 41.480 & 17.801 & 37.921 & 15.710 & 38.340 & 16.079 \\
\hline 4 & 19.238 & 11.158 & 19.933 & 10.514 & 19.334 & 10.586 \\
\hline 5 & 9.459 & 6.879 & 10.387 & 7.119 & 10.316 & 7.356 \\
\hline $\mathrm{N}$ & \multicolumn{2}{|c|}{7064} & \multicolumn{2}{|c|}{7159} & \multicolumn{2}{|c|}{6433} \\
\hline
\end{tabular}

\section{Table 2: Summary Statistics for Forecast Means, Subjective Uncertainty, and Realizations}

\begin{tabular}{|c|c|c|c|c|c|c|c|c|}
\hline Firm-Level Variables & $\begin{array}{l}(1) \\
\mathbf{N}\end{array}$ & $\begin{array}{c}(2) \\
\text { mean }\end{array}$ & $\begin{array}{l}\text { (3) } \\
\text { sd }\end{array}$ & $\begin{array}{l}\text { (13) } \\
\text { p10 }\end{array}$ & $\begin{array}{l}\text { (14) } \\
\text { p25 }\end{array}$ & $\begin{array}{l}\text { (15) } \\
\text { p50 }\end{array}$ & $\begin{array}{l}(16) \\
\text { p75 }\end{array}$ & $\begin{array}{l}(17) \\
\text { p90 }\end{array}$ \\
\hline Mean Employment Growth Rate Forecast, Next 12 Months & 7,064 & 0.009 & 0.080 & -0.050 & -0.011 & 0.006 & 0.033 & 0.078 \\
\hline Employment Growth Rate Uncertainty, Next 12 Months & 7,067 & 0.058 & 0.064 & 0.014 & 0.022 & 0.038 & 0.065 & 0.117 \\
\hline Realized Employment Growth Rate, Next 12 Months & 3,871 & 0.023 & 0.162 & -0.133 & -0.043 & 0.011 & 0.082 & 0.187 \\
\hline Mean Sales Growth Rate Forecast, Next 4 Quarters & 7,159 & 0.041 & 0.079 & -0.016 & 0.011 & 0.035 & 0.067 & 0.118 \\
\hline Sales Growth Rate Uncertainty, Next 4 Quarters & 7,160 & 0.044 & 0.048 & 0.010 & 0.016 & 0.028 & 0.052 & 0.095 \\
\hline Realized Sales Growth Rate, Next 4 Quarters & 3,091 & 0.050 & 0.262 & -0.250 & -0.063 & 0.047 & 0.175 & 0.353 \\
\hline Mean Expected Investment Rate (I/K), 4 Quarters Ahead & 6,433 & 0.115 & 0.296 & 0.005 & 0.013 & 0.035 & 0.088 & 0.240 \\
\hline Uncertainty about Investment Rate $(\mathrm{I} / \mathrm{K}), 4$ Quarters Ahead & 6,432 & 0.042 & 0.105 & 0.002 & 0.005 & 0.013 & 0.033 & 0.086 \\
\hline Realized Investment Rate (I/K), 4 Quarters Ahead & 7,186 & 0.091 & 0.253 & 0.001 & 0.008 & 0.024 & 0.067 & 0.180 \\
\hline Current Sales (Millions of Dollars) & 7,377 & 35.738 & 106.600 & 0.735 & 2.625 & 7.500 & 21.300 & 74.450 \\
\hline Current Employment & 17,387 & 401.185 & 999.226 & 17.000 & 58.000 & 139.000 & 288.000 & 700.000 \\
\hline Employment Growth Rate, Past 12 Months (Reported) & 7,488 & 0.021 & 0.123 & -0.095 & -0.018 & 0.017 & 0.069 & 0.143 \\
\hline
\end{tabular}

Notes: This table reports summary statistics computed using data from SBU survey waves between 10/2014 and 10/2019. We winsorized the firm-level variables at the 1st and 99th percentiles before computing the summary statistics. 


\section{Table 3: What fraction of respondents update their probability distributions for a given outcome between nearest same-topic surveys?}

\begin{tabular}{llc}
\hline Respondents Revising Their: & Fraction (SE) & N \\
\hline Vector of Probabilities for Employment 12 Months Ahead & $0.947(0.005)$ & 4,665 \\
Vector of Probabilities for Sales Growth Next 4 Quarters & $0.947(0.005)$ & 4,786 \\
Vector of Probabilities for Investment 4 Quarters Ahead & $0.947(0.006)$ & 4,639 \\
Vector of Support Points for Employment 12 Months Ahead & $0.959(0.004)$ & 4,665 \\
Vector of Support Points for Sales Growth Next 4 Quarters & $0.957(0.004)$ & 4,786 \\
Vector of Support Points for Investment 4 Quarters Ahead & $0.971(0.004)$ & 4,639 \\
\hline Employment Growth Expectations for Next 12 months & $0.998(0.001)$ & 4,519 \\
Employment Growth Uncertainty for Next 12 months & $0.998(0.001)$ & 4,519 \\
Sales Growth Expectations for Next 4 quarters & $0.995(0.002)$ & 4,757 \\
Sales Growth Uncertainty for Next 4 quarters & $0.995(0.002)$ & 4,757 \\
Investment Rate Expectations for 4 quarters ahead & $0.992(0.002)$ & 4,552 \\
Investment Rate Uncertainty for 4 quarters ahead & $0.995(0.002)$ & 4,552 \\
\hline
\end{tabular}

Notes: The top half of the table reports the fraction of respondents who provide different probabilities or support points between nearest sametopic surveys (i.e., in month $\mathrm{t}$ relative to month $t-2$ or $t-3$ ) for each of the three topics covered by the SBU. The bottom half reports the fraction of respondents whose subjective expectations and subjective uncertainty measures change between nearest same-topic surveys. Standard errors in parentheses, clustered by firm. The sample includes all survey waves from 10/2014 to 10/2019.

\section{Table 4: Cosine similarity between responses in nearest same-topic surveys}

\begin{tabular}{lcc}
\hline Vectors of Responses & $\begin{array}{c}\text { Mean Cosine Similarity } \\
\text { between vectors reported in } \\
\text { months } \boldsymbol{t} \text { and } \boldsymbol{t}+\mathbf{2}(\mathbf{o r} \boldsymbol{t}+\mathbf{3}) \text { (SE) }\end{array}$ & N \\
\hline Vector of Probabilities for Employment 12 Months Ahead & $0.883(0.004)$ & 4,661 \\
Vector of Probabilities for Sales Growth Next 4 Quarters & $0.884(0.004)$ & 4,786 \\
Vector of Probabilities for Investment 4 Quarters Ahead & $0.878(0.004)$ & 4,639 \\
Vector of Support Points for Employment Growth 12 Months Ahead & $0.844(0.005)$ & 4,500 \\
Vector of Support Points for Sales Growth Next 4 Quarters & $0.885(0.053)$ & 4.715 \\
Vector of Support Points for Investment 4 Quarters Ahead & $0.976(0.008)$ & 4,634 \\
\hline
\end{tabular}

Notes: This table reports the mean cosine similarity across the response vectors respondents provide in consecutive survey waves, i.e. the cosine between the vector provided in month $t$ and the vector provided in month $t+2$ or $t+3$ when the respondent next receives the survey for the same topic. For each pair of consecutive responses for a given topic, we compute the cosine similarity between the vectors of probabilities and outcomes the respondent provides, and then we compute the mean cosine similarity. Standard errors are in parentheses, clustered by firm. The sample includes all SBU responses between 10/2014 and 10/2019. 


\section{Table 5: Autocorrelations of Growth Rate Expectations and Uncertainty}

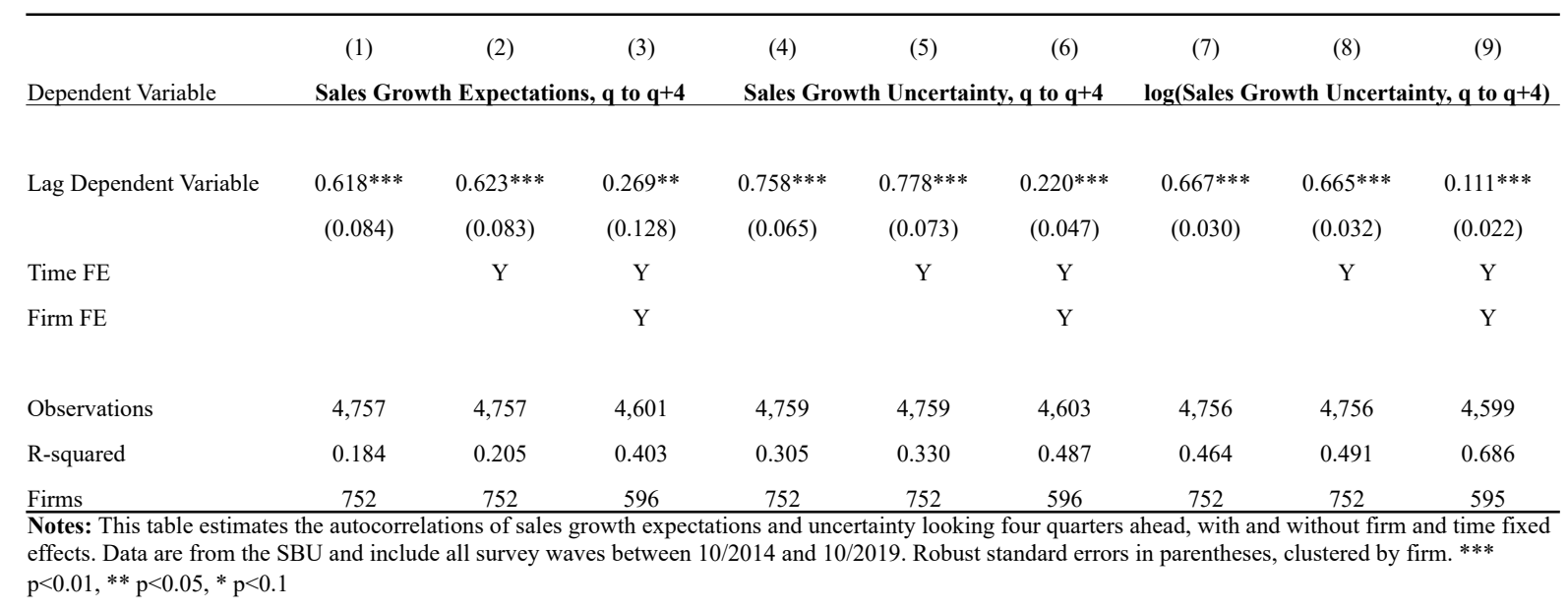

\section{Table 6: Subjective Uncertainty Is Unaffected by the Number of Previous Survey Completions}

\begin{tabular}{|c|c|c|c|c|c|c|}
\hline \multirow{4}{*}{$\begin{array}{l}\text { Dependent Variable } \\
\text { No. of Previous Responses }\end{array}$} & (1) & (2) & (3) & (4) & (5) & (6) \\
\hline & \multicolumn{6}{|c|}{ log of Subjective Uncertainty over: } \\
\hline & \multicolumn{2}{|c|}{$\begin{array}{l}\text { Sales Growth, } \\
\text { Next } 4 \text { Quarters }\end{array}$} & \multicolumn{2}{|c|}{$\begin{array}{l}\text { Employment Growth, } \\
\text { Next } 12 \text { Months }\end{array}$} & \multicolumn{2}{|c|}{$\begin{array}{l}\text { Investment Rate, } \\
4 \text { Quarters Ahead }\end{array}$} \\
\hline & $\begin{array}{l}-0.004 \\
(0.004)\end{array}$ & $\begin{array}{l}-0.003 \\
(0.005)\end{array}$ & $\begin{array}{l}-0.001 \\
(0.003)\end{array}$ & $\begin{array}{l}-0.005 \\
(0.004)\end{array}$ & $\begin{array}{c}0.005 \\
(0.009)\end{array}$ & $\begin{array}{l}-0.015 \\
(0.012)\end{array}$ \\
\hline Firm FE & $\mathrm{Y}$ & Y & $\mathrm{Y}$ & $\mathrm{Y}$ & $\mathrm{Y}$ & $\mathrm{Y}$ \\
\hline Time FE & Y & $\mathrm{Y}$ & $\mathrm{Y}$ & Y & Y & $\mathrm{Y}$ \\
\hline Employment-weighted & & Y & & $\mathrm{Y}$ & & $\mathrm{Y}$ \\
\hline Mean of Dependent Variable & -3.541 & -3.711 & -3.245 & -3.497 & -4.371 & -4.474 \\
\hline SD of Dependent Variable & 0.880 & 0.807 & 0.834 & 0.773 & 1.537 & 1.427 \\
\hline Observations & 6,791 & 6,588 & 6,712 & 6,703 & 6,196 & 6,042 \\
\hline Within R-squared & 3.04E-04 & $2.22 \mathrm{E}-04$ & $7.62 \mathrm{e}-05$ & 7.01E-04 & $1.85 \mathrm{E}-04$ & $1.21 \mathrm{E}-03$ \\
\hline R-squared & 0.709 & 0.695 & 0.796 & 0.742 & 0.707 & 0.681 \\
\hline
\end{tabular}




\title{
Figure 1: SBU Excerpts, September 2016 Onwards
} 1a. Employment Questions

\section{SBU Survey of Business Uncertainty}

\author{
翼, PEDERAL RESERVE BANK of ATLANTA \\ CHICAROBOOTH: \\ Stanford
}

Currently, what is your NUMBER OF EMPLOYEES (including part-time)?

Looking back, 12 months ago, what was your NUMBER OF EMPLOYEES

(including part-time)?

\section{SBU Survey of Business Uncertainty}

\section{SBU Survey of Business Uncertainty}

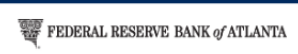
CHICAROBOOTH

$\underset{\text { University }}{\text { Stanford }}$

Looking ahead, 12 months from now, what NUMBER OF EMPLOYEES (including part-time) would you assign to each of the following scenarios?

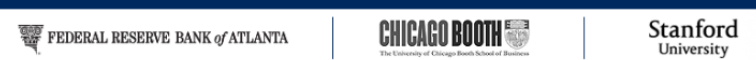

Please assign a percentage likelihood to the NUMBER OF EMPLOYEES you entered above. (Values should sum to $100 \%$ ) 


\section{1b. Sales Questions}

\section{SBU Survey of Business Uncertainty}

\begin{tabular}{|c|c|}
\hline 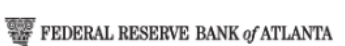 & 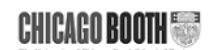 \\
\hline
\end{tabular}

For the current quarter, what would you estimate the total dollar value of your SALES REVENUE will be?

$\$$

Looking back, over the last 12 months, what was your approximate percentage SALES REVENUE GROWTH rate?

\section{SBU Survey of Business Uncertainty}

嫼 PEDERAL RESBRVE BANK f ATLANTA

CHICACOBOOHF:

$\underset{\text { University }}{\text { Stanford }}$

Looking ahead, from now to four quarters from now, what approximate percentage SALES REVENUE growth rate would you assign to each of the following scenarios?

\begin{tabular}{|c|c|}
\hline The LOWEST percentage sales revenue growth rate would be about: & -2 \\
\hline A LOW percentage sales revenue growth rate would be about: & -1 \\
\hline A MIDDLE percentage sales revenue growth rate would be about: & 0 \\
\hline A HIGH percentage sales revenue growth rate would be about: & 1 \\
\hline The HIGHEST percentage sales revenue growth rate would be about: & 2 \\
\hline
\end{tabular}

Please assign a percentage likelihood to the SALES REVENUE growth rates you entered. (Values should sum to 100\%)

LOWEST: The likelihood of realizing a $\mathbf{- 2} \%$ sales revenue growth rate would be: LOW: The likelihood of realizing a $-1 \%$ sales revenue growth rate would be: MIDDLE: The likelihood of realizing a $0 \%$ sales revenue growth rate would be: HIGH: The likelihood of realizing a $1 \%$ sales revenue growth rate would be: HIGHEST: The likelihood of realizing a $\mathbf{2} \%$ sales revenue growth rate would be Total 


\section{Figure 2: Subjective Sales Growth Rate Expectations Predict Realized Growth Rates}

Figure 2a

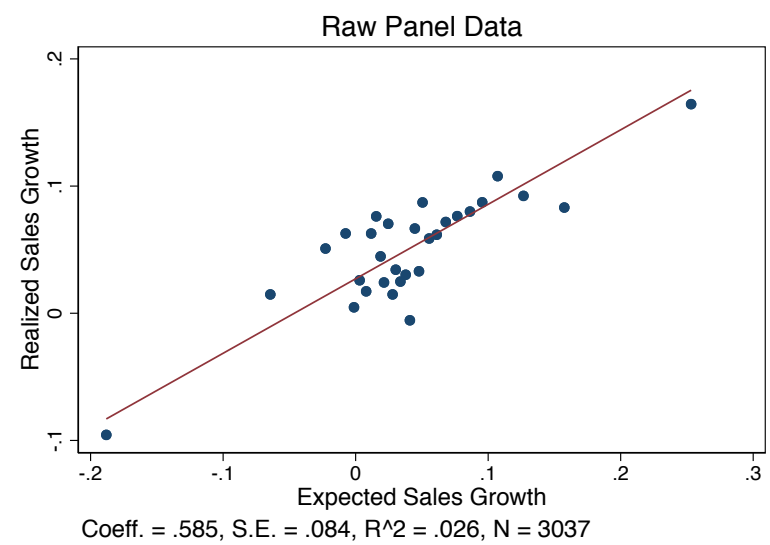

Figure 2c

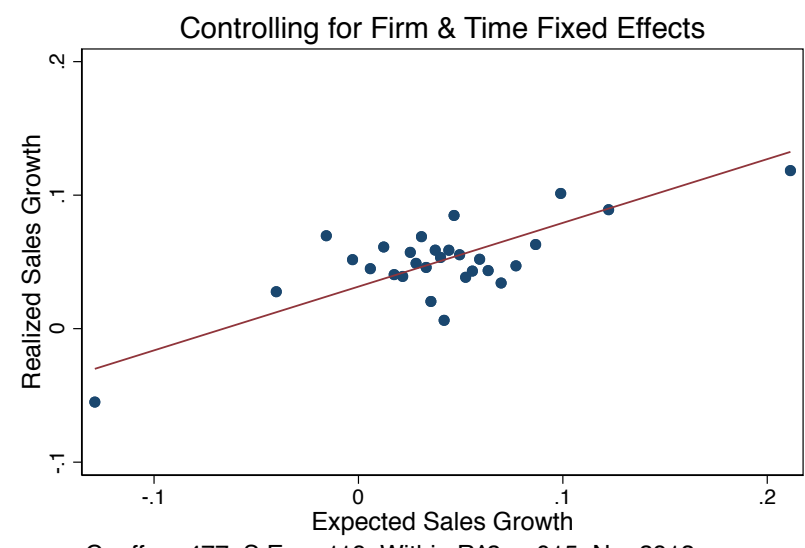

Figure $2 b$

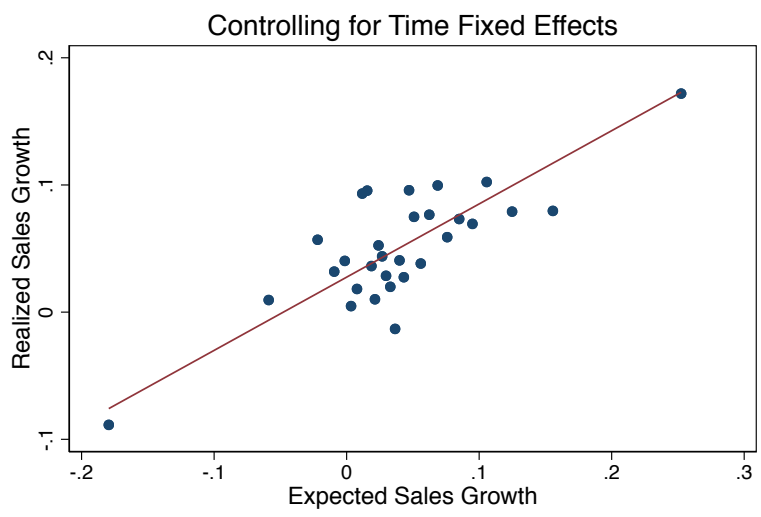

Coeff. $=.575$, S.E. $=.086$, Within $\mathrm{R}^{\wedge} 2=.024, \mathrm{~N}=3037$

Coeff. $=.477$, S.E. $=.119$, Within $\mathrm{R}^{\wedge} 2=.015, \mathrm{~N}=2913$

Figure 2d

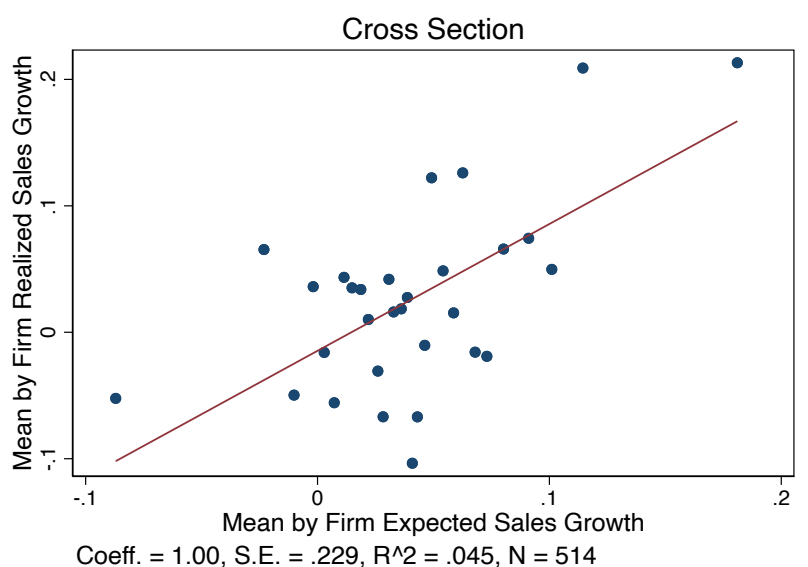

Notes: This figure shows bin-scatter plots of sales growth rate expectations for the next 4 quarters on the horizontal axis against realized sales growth rates over the ensuing 4 quarters on the vertical axis. Figure $2 \mathrm{a}$ shows the relationship in the raw panel data. Figure $2 \mathrm{~b}$ controls for time effects. Figure $2 c$ controls for both firm and time fixed effects. Figure $2 d$ shows the relationship in the cross section, showing the meanby-firm expected sales growth on the horizontal axis and mean-by-firm realized sales growth on the vertical axis. The reported statistics below each figure figure correspond to the OLS regression in the underlying micro data, reporting firm-clustered standard errors. Data are from all waves of the SBU from 10/2014 to 10/2019. 


\section{Figure 3: Subjective Uncertainty Predicts Absolute Forecast Errors}

Figure 3a

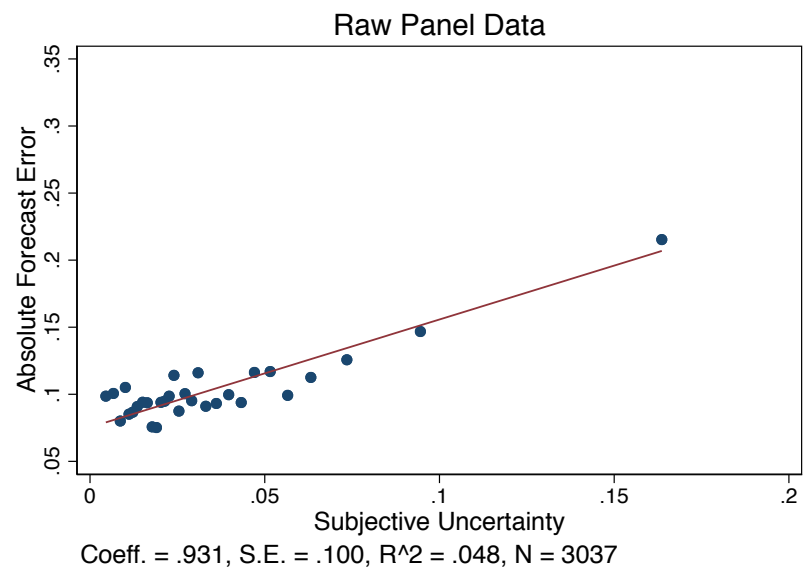

Figure 3c

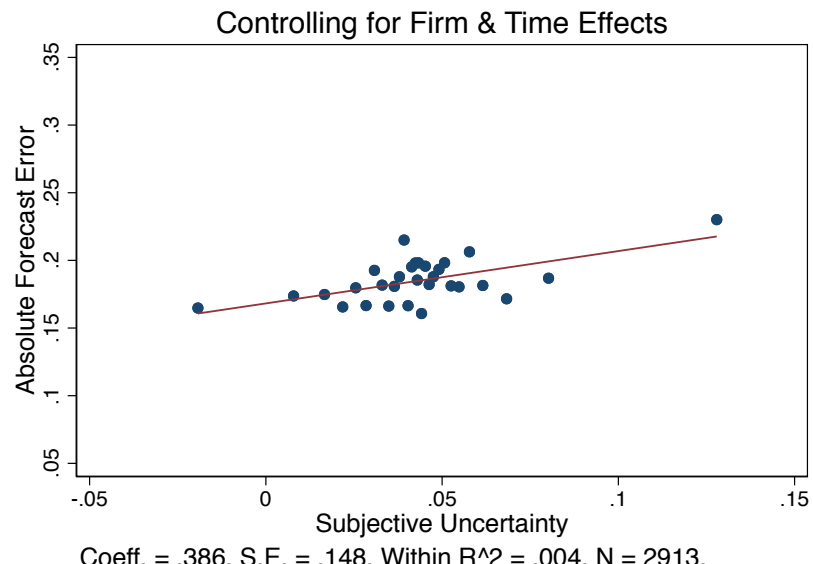

Figure $3 b$

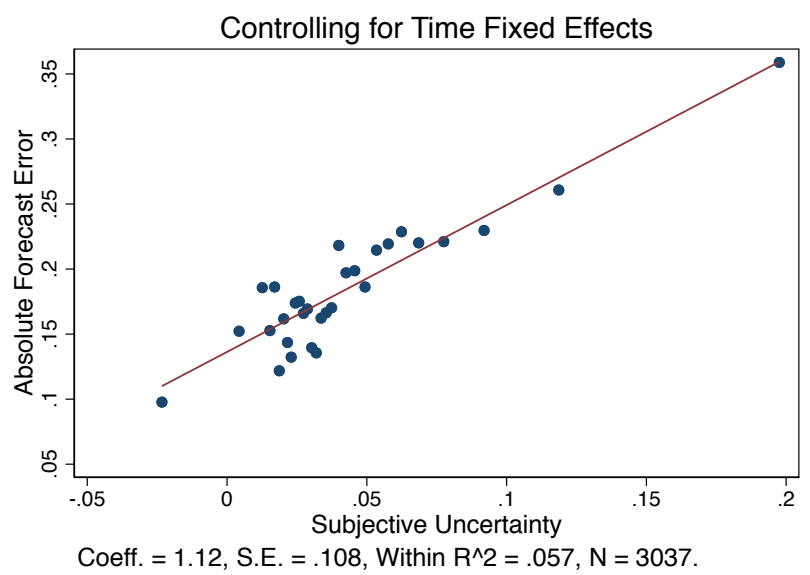

Figure 3d

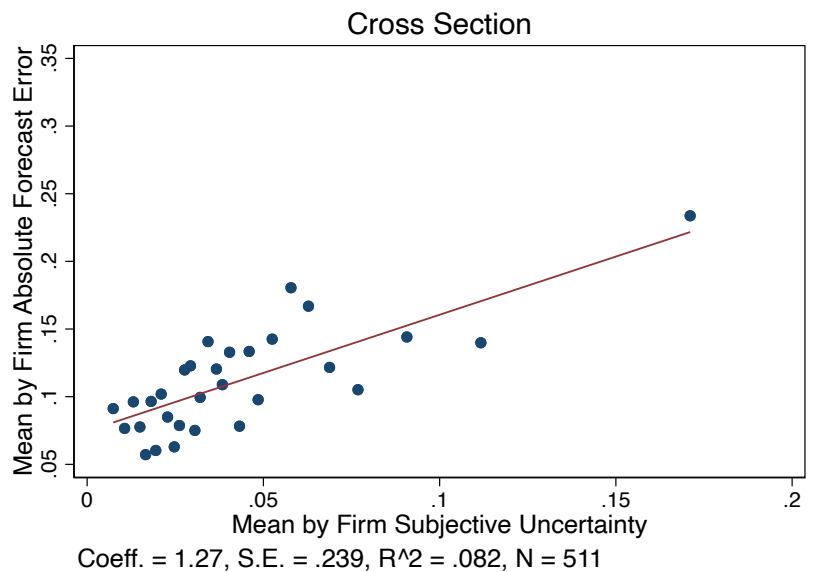

Notes: This figure shows a bin-scatter plots of subjective uncertainty about the firm's sales growth rate for the next 4 quarters on the horizontal axis, against the respondent's absolute forecast error for its sales growth rate over the ensuing 4 quarters on the vertical axis. Figure $3 a$ shows the relationship in the raw panel. Figure $3 \mathrm{~b}$ controls for time effects. Figure $3 \mathrm{c}$ also controls for firm effects. Figure $3 \mathrm{~d}$ shows the relationship in the cross section, plotting mean-by-firm subjective uncertainty on the horizontal axis against the mean-by-firm absolute forecast error on the vertical axis. The statistics below each figure correspond to the OLS regression in the underlying micro data, reporting firm-clustered standard errors. Data are from all waves of the SBU from 10/2014 to 10/2019. 


\section{Figure 4: Uncertainty and subsequent expectation revisions}

Figure 4a.

Raw Panel Data

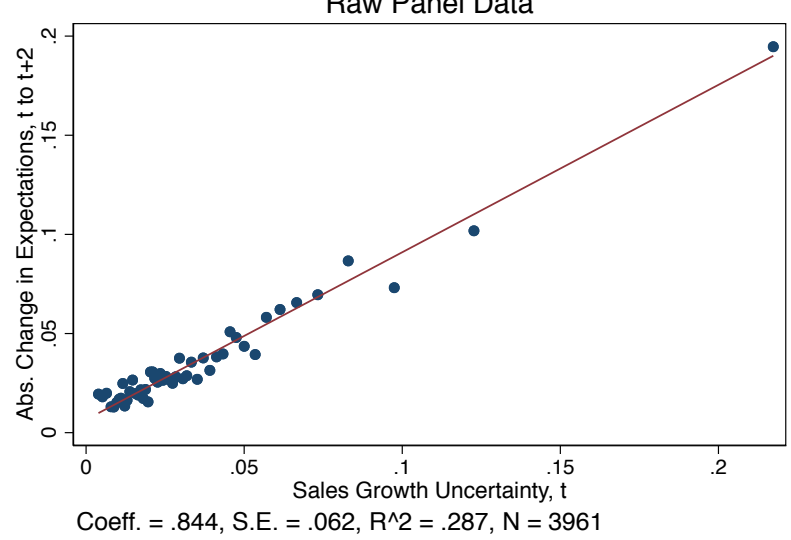

Figure $4 b$.

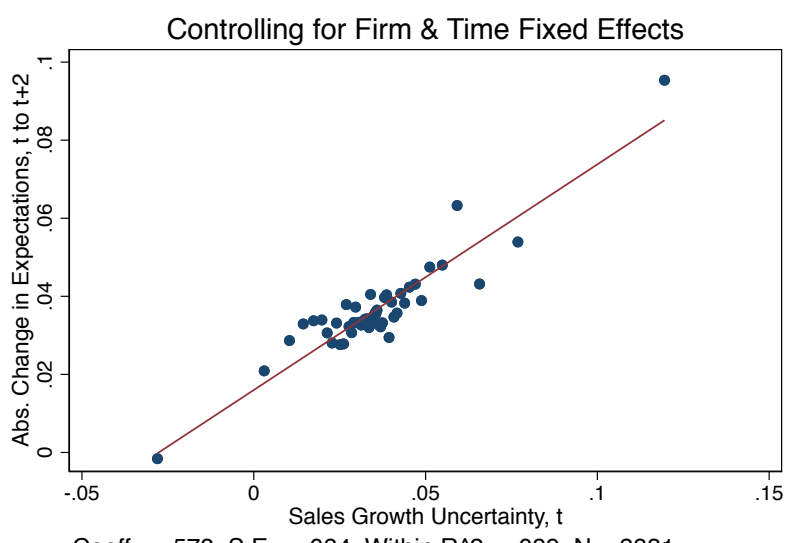

Coeff. $=.578$, S.E. $=.084$, Within $\mathrm{R}^{\wedge} 2=.089, \mathrm{~N}=3831$

Notes: This figure shows two bin-scatter plots. On the horizontal axis, both show 50 quantiles of subjective uncertainty for sales growth rates over the next four quarters, measured in month $t$. Both have on the vertical axis the absolute value of the change in sales growth rate expectations from months $t$ to $t+2$ (or $t+3$ ). On the left, we show the relationship in the raw panel data, while on the right we show the relationship controlling for firm and time fixed effects. We report the underlying firm-level regressions with firm-clustered standard errors at the bottom of each figure, using SBU data from 10/2014 to 10/2019.

\section{Figure 5: Uncertainty and subsequent uncertainty revisions}

Figure 5a.

Raw Panel Data

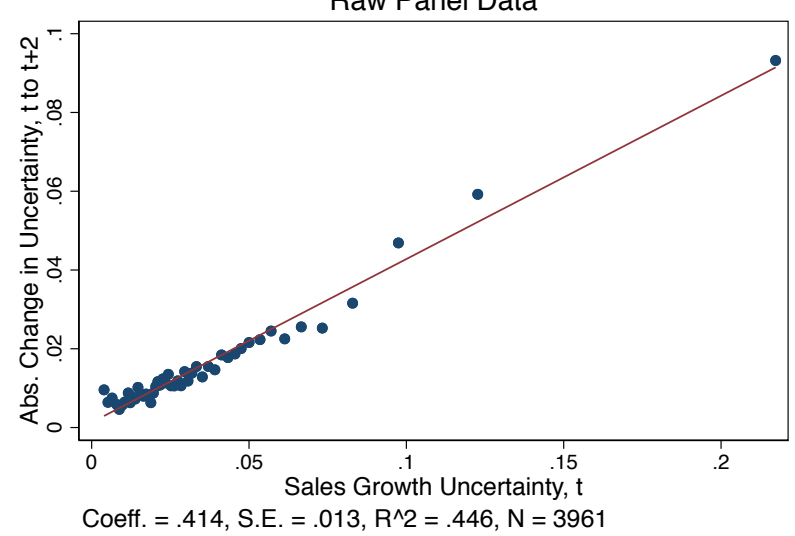

Figure 5b.

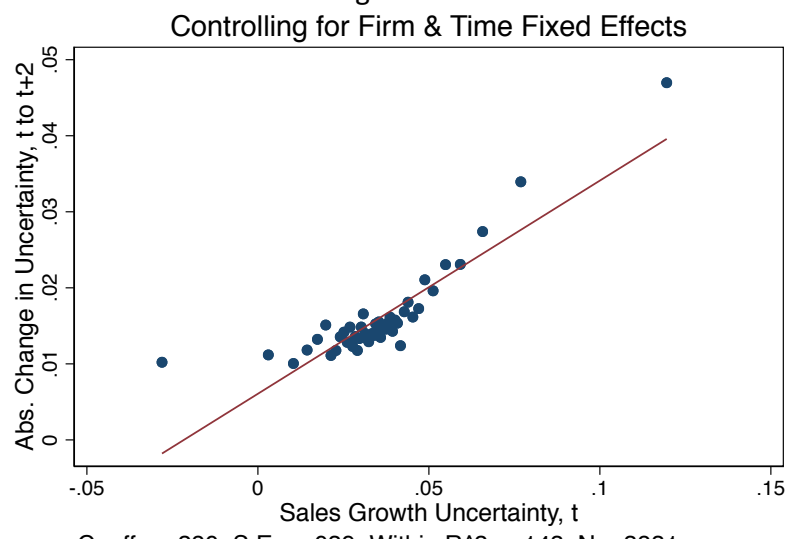

Coeff. $=.280$, S.E. $=.039$, Within $\mathrm{R}^{\wedge} 2=.143, \mathrm{~N}=3831$

Notes: This figure shows two bin-scatter plots. On the horizontal axis, both show 50 quantiles of subjective uncertainty for sales growth rates over the next four quarters, measured in month $t$. Both have on the vertical axis the absolute value of the change in sales growth rate uncertainty from month $t$ to $t+2$ (or $t+3$ ). On the left, we show the relationship in the raw panel data, while on the right we show the relationship controlling for firm and time fixed effects. We report the underlying firm-level regressions with firm-clustered standard errors at the bottom of each figure, using SBU data from 10/2014 to 10/2019. 


\section{Figure 6: Subjective uncertainty has a V-shaped relationship to past sales growth and recent forecast revisions \\ Figure 6a. \\ Figure 6b.}

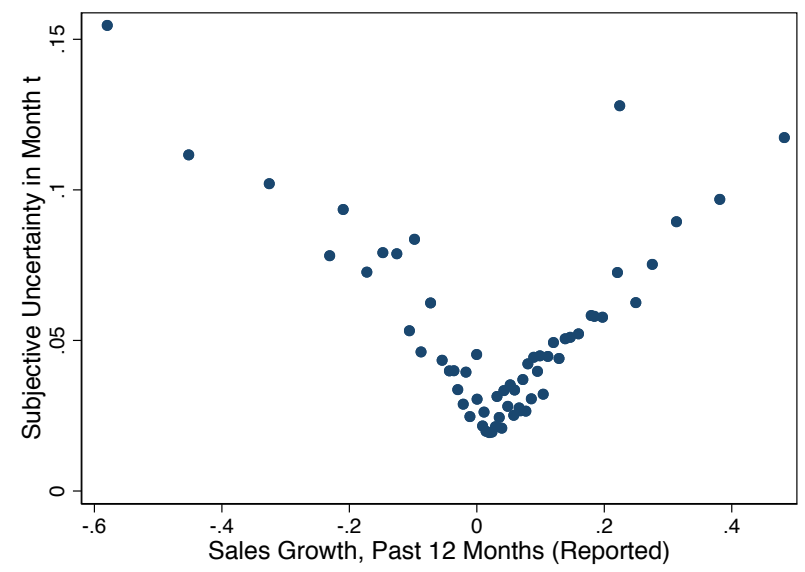

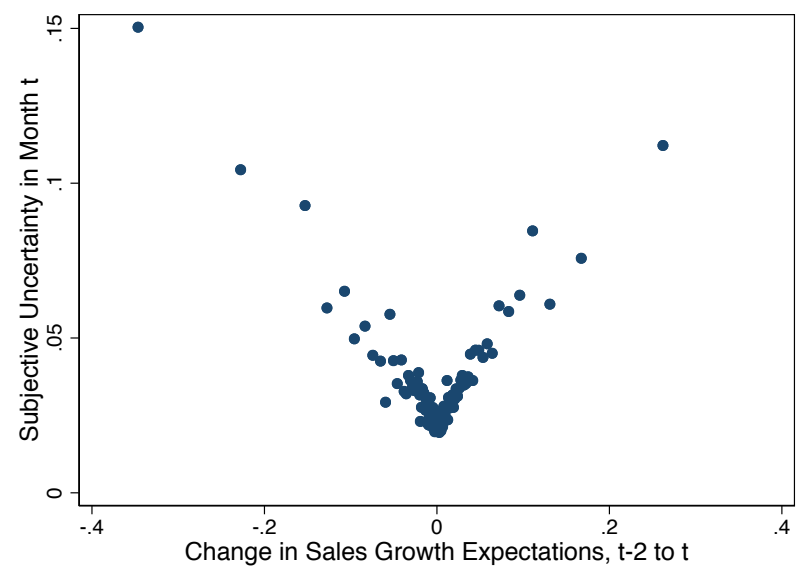

Notes: This figure shows two bin-scatter plots with subjective uncertainty over four-quarter-ahead sales growth rates at $t$ on the vertical axis. Figure 6a shows shows 100 quantiles of past sales growth rate from month $t-12$ to $t$ on the horizontal axis. Figure $6 \mathrm{~b}$ instead shows 100 quantiles of the change in the four-quarter-ahead sales growth rate expectations from $t-2$ (or $t-3$ ) to $t$. Data are from the SBU and the sample covers all survey waves from 10/2014 to 10/2019.

\section{Figure 7: Business Expectations and}

Figure 7a.

Business Expectations Indices

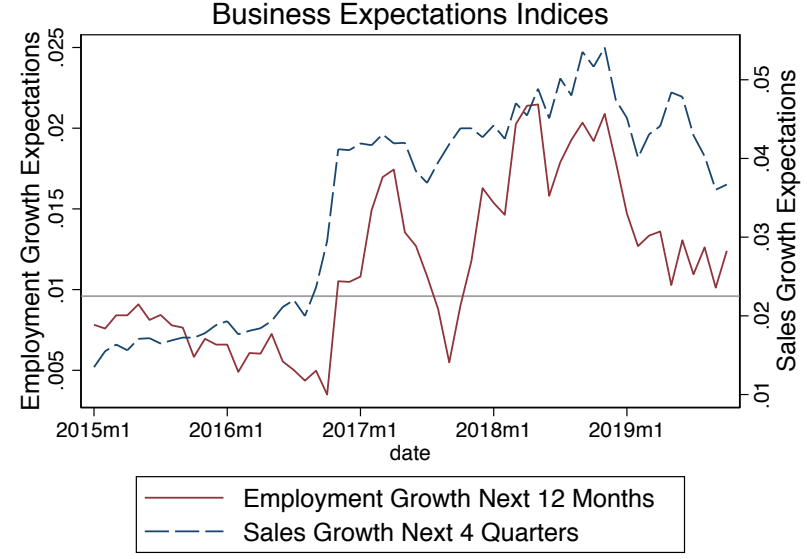

Figure 7b.

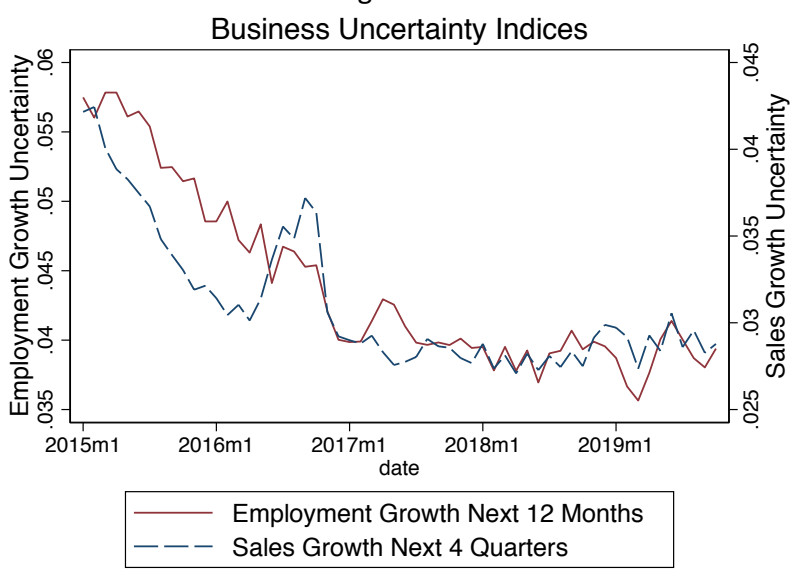

Notes: The above figures show business expectations (left) and business uncertainty (right) indices the employment growth rate $\mathrm{n}$ the next 12 months and the sales growth rate in the next 4 quarters. 


\section{Figure 8: External comparisons}

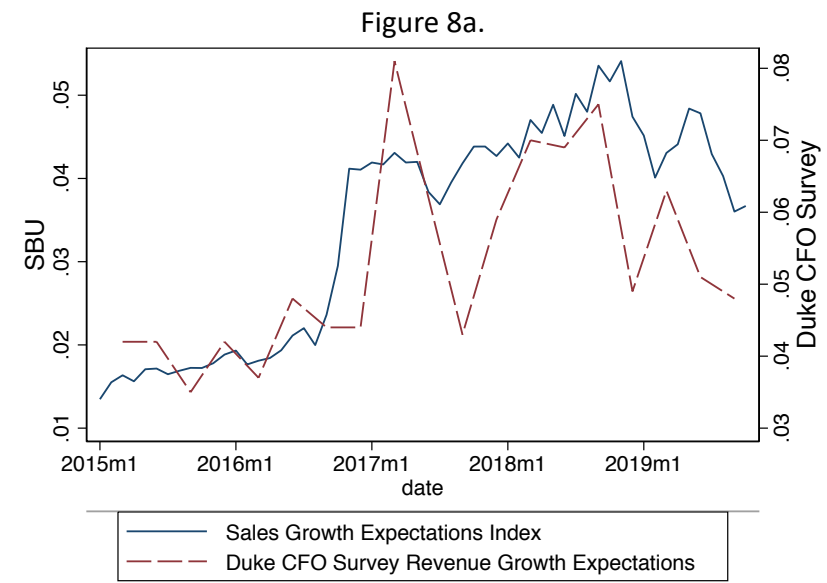

Correlation: .723

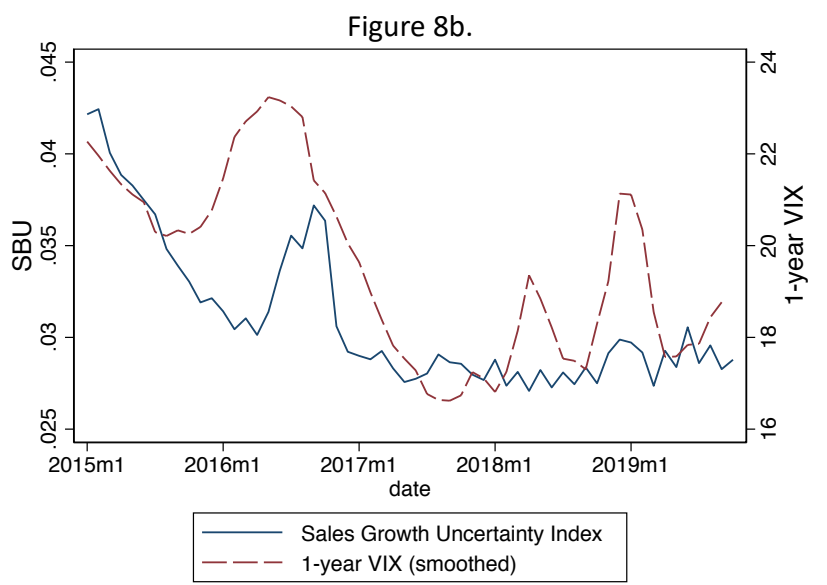

Correlation: .674

Notes: The left panel shows our Sales Growth Rate Expectations Index and the revenue-weighted average of expected change in revenue growth rates for the next 12 months among firms answering the Duke CFO Survey. Because the Duke Survey goes to field in the third month of each quarter, we align its quarterly responses to March, June, September and December. The right panel shows our Sales Growth Rate Uncertainty Index and the 1-year VIX on the 15th day of each month (Source: CBOE via Bloomberg). If the 15th is not a trading day we try the 16 th, 14 th, 17 th, 13th, 18th, or 12th in that order. We smooth the monthly 1-year VIX series using the same procedure as for our Business Uncertainty Index.

\section{Figure 9: Skewness in realized future growth rates rises with current subjective skewness}

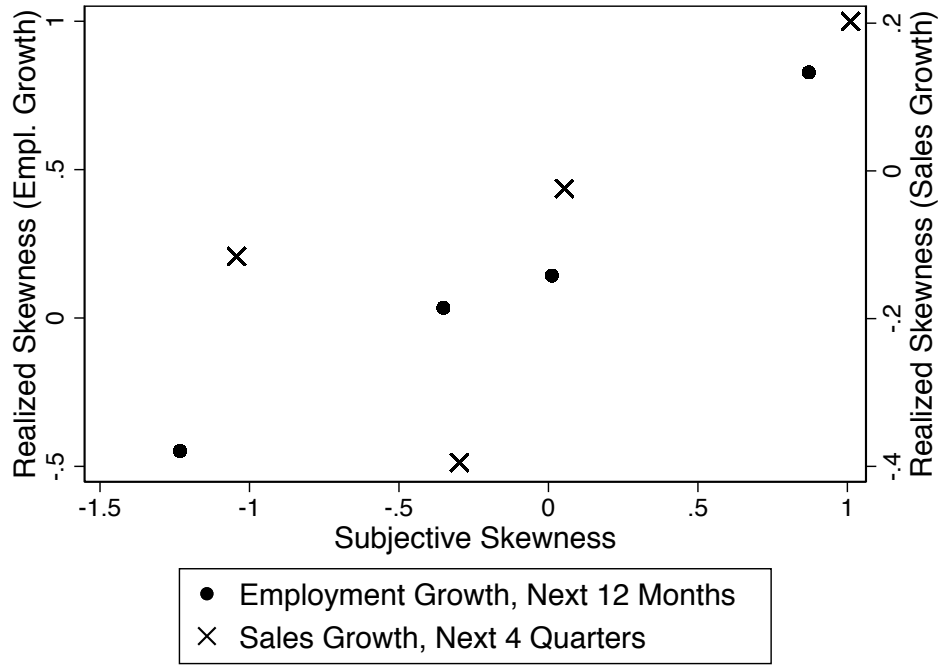

Notes: We sort firm-level values for subjective skewness over future sales growth rates into quartiles, and we do the same for subjective skewness over future employment growth rates. For each quartile, we then compute the average subjective skewness values and the skewness of realized employment or sales growth rates over the forecast horizon. The chart displays these quartile-specific values for sales and employment growth rates.

$\mathrm{N}($ Employment $)=3,692$. $\mathrm{N}($ Sales $)=$ 3,037. 


\section{Figure 10: Previous Survey Completions and Subjective Uncertainty \\ Figure 10a}

Dep Variable: $\log$ (Sales Growth Uncertainty)

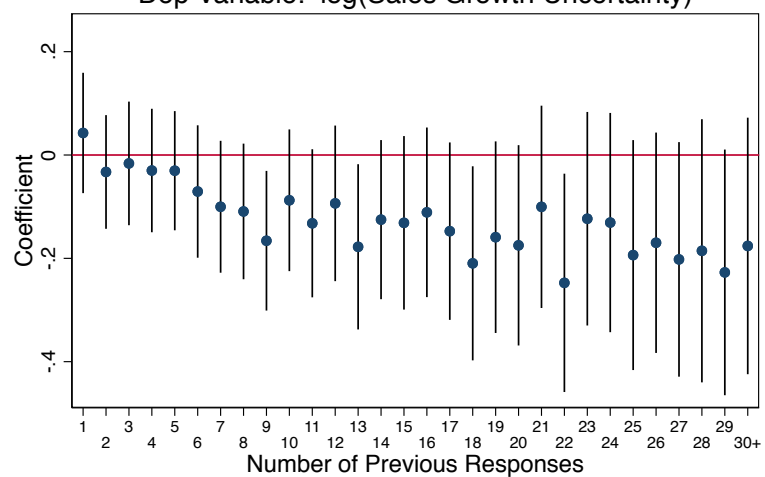

Mean (SD) of Dep. Var: $-3.54(.880)$

\author{
Dep Variable: log(Sales Growth Uncertainty)
}

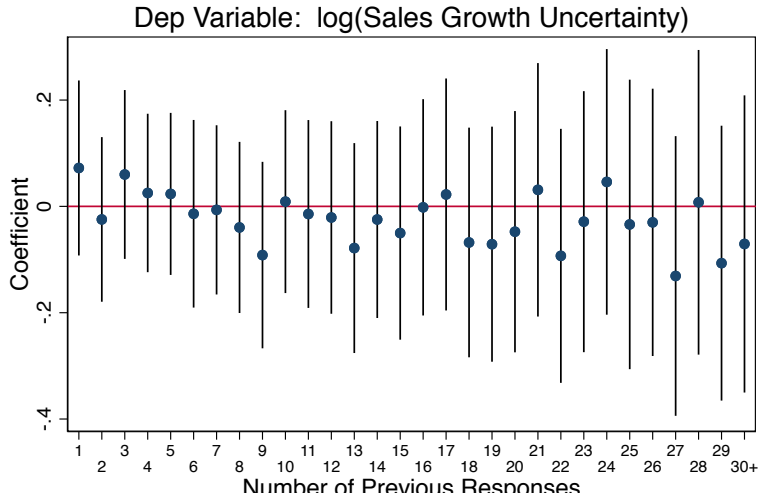

Mean (SD) of Dep. Var: -3.71 (.806). Employment-weighted

Notes: This figure shows estimated coefficients and 95 percent confidence intervals from regressions of the natural log of sales growth uncertainty (looking ahead over the next 4 quarters) on a set of indicators for the firm's number of previous SBU responses on the righthand-side as well as firm and time fixed effects (not shown). Figure 4a (left) shows unweighted estimates, while figure $4 \mathrm{~b}$ (right) weights observations by employment (winsorized at 500 employees). We top-code the number of responses at 30. Data are from the SBU and cover all survey waves between 10/2014 and 10/2019. We construct the 95 percent confidence intervals based on firm-clustered robust standard errors.

\section{Figure 11: Reinterpreting SBU responses as approximations to continuous distributions}
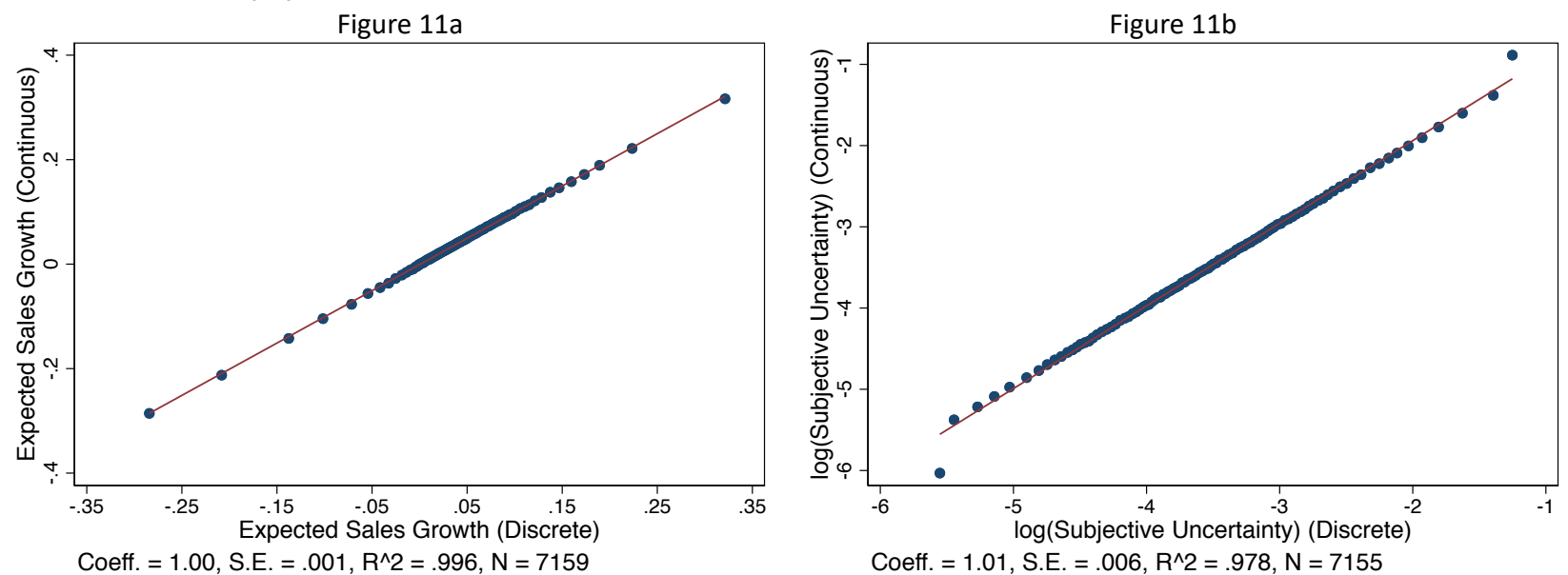

Notes: The figures show bin-scatter plots that compare our measures of subjective mean expectations and uncertainty interpreting SBU responses as discrete or subjective distributions. Our baseline measures interpret SBU responses as discrete, 5-point probability distributions. Alternatively, we can interpret the responses as a continuous distribution consisting of 5 bins, with a uniform distribution within each bin. Figure 12 a plots 100 percentiles of our discrete measure of expected sales growth (looking four quarters ahead) on the horizontal axis against the continuous measure of expectations on the vertical axis. Figure $12 \mathrm{~b}$ repeats the exercise for the natural logarithm of subjective uncertainty. Statistics below the figure correspond to the OLS regression in the underlying microdata, reporting firm-clustered standard errors. Data are from all waves of the SBU from 10/2014 to 10/2019. 


\section{Figure 12: Sales growth expectations and uncertainty indices controlling for panel composition}
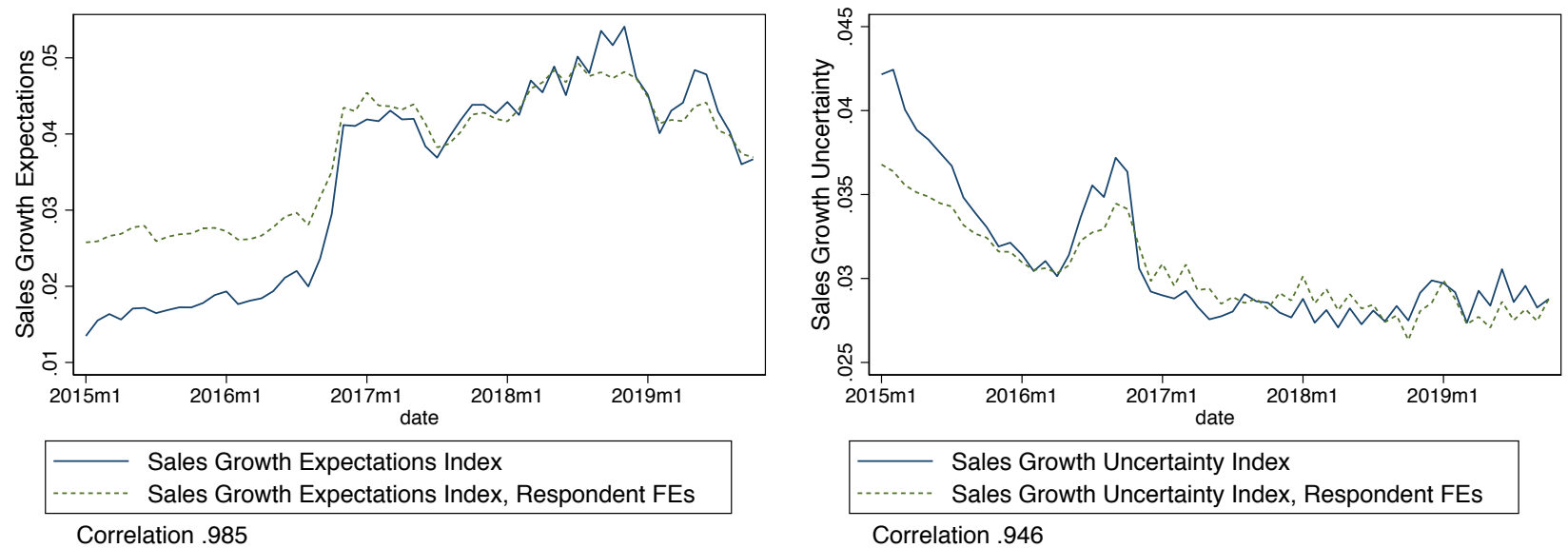

Notes: The figures plot our baseline sales growth rate expectations (left) and uncertainty (right) indices alongside an alternatives that account for changing panel composition across months. Our baseline index computes an activity-weighted mean for expectations or uncertainty in each month. By contrast, the alternative index computes the same activity weighted mean after controlling for respondent fixed effects. We smooth both indices using the same procedure. Data are from the SBU and cover all months between 1/2015 and 10/2019. 


\section{Surveying Business Uncertainty Online Appendices, Not Intended for Publication}

David Altig, Jose Maria Barrero, Nicholas Bloom, Steven J. Davis, Brent Meyer, and Nicholas Parker

March 2020 


\section{A. Additional Information about the SBU}




\title{
Figure A.1: Capital Expenditures Questionnaire, May 2019 - present
}

\author{
SBU Survey of Business Uncertainty

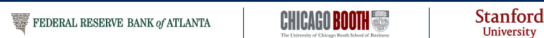 \\ Please provide an estimate of the book value of all property, \\ plant, and equipment owned by your firm.

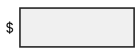 \\ For the current quarter, what would you estimate the total \\ dollar value of your capital investment expenditures will be?

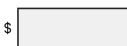 \\ Looking back, four quarters ago, what was the approximate \\ dollar value of your capital investment expenditures?

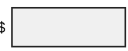

\section{SBU Survey of Business Uncertainty}

\begin{tabular}{c|c|c} 
䨳 FEDERAL RESERVE BANK of ATLANTA & CHICACOBOOTHE & $\begin{array}{c}\text { Stanford } \\
\text { University }\end{array}$
\end{tabular}

Looking ahead, from now to four quarters from now, what approximate dollar value of capital investment expenditures would you assign to each of the following scenarios?

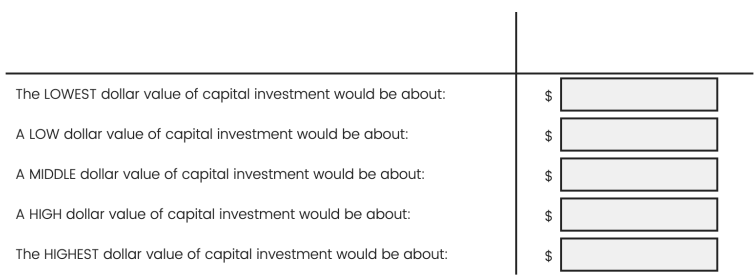

\section{SBU Survey of Business Uncertainty}

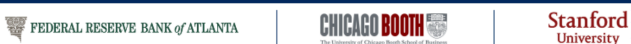

Please assign a percentage likelihood to the capital

investment expenditures you entered. (Values should sum to $100 \%)$

$$
\begin{aligned}
& \text { LOWEST CASE: The likelihood of about } \mathbf{\$ 1} \text { in capital investment expenditures } \\
& \text { would be: } \\
& \text { LOW CASE: The likelihood of about } \mathbf{\$ 2} \text { in capital investment expenditures } \\
& \text { would be: } \\
& \text { MIDDLE CASE: The likelihood of about } \mathbf{\$} 3 \text { in capital investment expenditures } \\
& \text { would be: } \\
& \text { HIGH CASE: The likelihood of about } \$ \mathbf{4} \text { in capital investment expenditures } \\
& \text { would be: } \\
& \text { HIGHEST CASE:The likelihood of about } \mathbf{\$ 5} \text { in capital investment } \\
& \text { expenditures would be: } \\
& \text { Total }
\end{aligned}
$$




\section{Figure A.2: Capital Expenditures Questionnaire, Prior to May 2019}

\section{SBU Survey of Business Uncertainty}

\begin{tabular}{|c|c|}
\hline 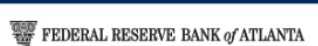 & CHICAROBOOTH: \\
\hline
\end{tabular}

For the current quarter, what would you estimate the total dollar value of your CAPITAL INVESTMENT expenditures will be?

Looking back, four quarters ago, what was the approximate dollar value of your CAPITAL INVESTMENT expenditures?

\section{SBU Survey of Business Uncertainty}

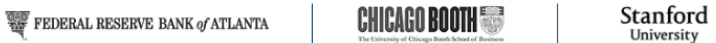

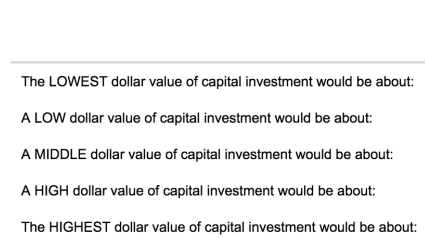




\title{
Figure A.3: Sales Question (in levels) During SBU $1^{\text {st }}$ Generation (August 2014 - August 2016)
}

\section{SBU Survey of Business Uncertainty}

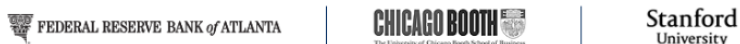 \\ In the current quarter, what is the approximate dollar value of your SALES \\ REVENUE?
}

$\$$

Looking back, four quarters ago, what was the approximate dollar value of your SALES REVENUE?

\section{SBU Survey of Business Uncertainty}

变 FEDERAL RESERVE BANK of ATLANTA

CHICAEOBOOTHE

Stanford

Looking ahead, from now to four quarters from now, what approximate dollar value of SALES REVENUE would you assign to each of the following scenarios?

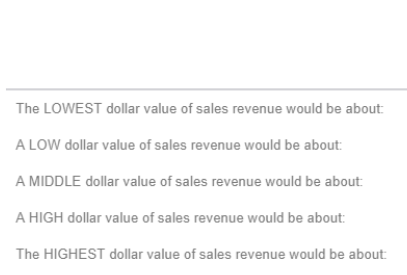

\begin{tabular}{l|}
$\$ \quad 5,000,000$ \\
$\$ \quad 6,000,000$ \\
$\$ \quad 7,000,000$ \\
$\$ \quad 8,000,000$ \\
$\$ \quad 9,000,000$
\end{tabular}

$\$ 5,000,000$

$\$ 7,000,000$

$\$ 9,000,000$

\section{SBU Survey of Business Uncertainty}

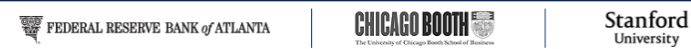

Please assign a percentage likelihood to the SALES REVENUE dollar values you entered. (Values should sum to $100 \%$ )

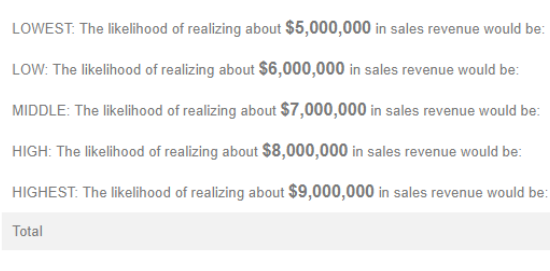




\section{A.1 Obtaining subjective moments (expectations and uncertainty) about future own-firm outcomes from the raw survey data}

- The next few slides slides explain how we use the survey responses to compute moments of subjective probability distributions over own-firm future outcomes.

- We calculate first and second moments of the subjective growth rate distributions of employment, sales and unit costs over the next 12 months or four quarters, as appropriate.

- Following standard practice in the literature on business-level dynamics, we calculate the growth rate of $x$ from $t-1$ to $t$ as $g_{t}=2\left(x_{t}-x_{t-1}\right) /$ $\left(x_{t}+x_{t-1}\right)$.

- For capital investment, we calculate first and second moments of the subjective distribution for future investment rate (I/K).

* This definition of the growth rate of sales is convenient for its symmetry around zero and because its support lies on the closed interval $[-2,2]$, with the endpoints of the interval corresponding to entry and exit. See "Gross Job Creation, Gross Job Destruction, and Employment Reallocation" by Steven J. Davis and John Haltiwanger in the 1992 Quarterly Journal of Economics for a more extensive discussion.

\section{A.1.1 Employment}

Respondent Data

$C E m p=$ firm's current employment level, as reported by the respondent

$F E m p_{i}=$ employment 12 months hence, $i=1,2,3,4,5$

$p_{i}=$ the associated probabilities, $i=1,2,3,4,5$

Scenario-Specific Growth Rates

$E G r_{i}=2\left(F E m p_{i}-C E m p\right) /\left(F E m p_{i}+C E m p\right), i=1,2,3,4,5$

First and Second Moments of the Subjective Growth Rate Distribution

$\operatorname{Mean}(E G r)=\sum_{i=1}^{5} p_{i} E G r_{i}$

$\operatorname{Var}(E G r)=\sum_{i=1}^{5} p_{i}\left(E G r_{i}-\operatorname{Mean}(E G r)\right)^{2}$

$S D(E G r)=\sqrt{\operatorname{Var}(E G r)}$ 


\section{A.1.2 Sales Revenue (Current SE Questionnaire)}

Respondent Data

CSale $=$ firm's sales revenue in the current quarter, as reported by the respondent

$F$ Sale $G r_{i}=$ respondent's scenario-specific sales growth rate from now to four quarters hence, $i=$ $1,2,3,4,5$

$p_{i}=$ the associated probabilities, $i=1,2,3,4,5$

Implied Future Sales Level

FSale $_{i}=\left(1+\frac{\text { FSaleG }}{100}\right)$ CSale $, i=1,2,3,4,5$

Scenario-Specific Growth Rates (re-expressing respondent growth rates to our growth rate measure)

SaleGr $_{i}=2\left(\right.$ FSale $_{i}-$ CSale $) /\left(\right.$ FSale $_{i}+$ CSale $)=2$ FSaleGr $_{i} /\left(\right.$ FSaleGr $\left._{i}+2\right), i=1,2,3,4,5$

First and Second Moments of the Subjective Growth Rate Distribution

Mean $($ SaleGr $)=\sum_{i=1}^{5} p_{i}$ SaleGr $_{i}$

$\operatorname{Var}($ SaleGr $)=\sum_{i=1}^{5} p_{i}\left(\text { SaleGr }_{i}-\text { Mean }(\text { SaleGr })_{i}\right)^{2}$

$S D($ SaleGr $)=\sqrt{\text { Var(SaleGr })}$

\section{A.1.3 Sales Revenue (Old SE Questionnaire) \\ Respondent Data \\ CSale $=$ firm's sales revenue in the current quarter, as reported by the respondent \\ FSale $_{i}=$ sales revenue four quarters hence, $i=1,2,3,4,5$ \\ $p_{i}=$ the associated probabilities, $i=1,2,3,4,5$}

Scenario-Specific Growth Rates

SaleGr $_{i}=2\left(\right.$ FSale $_{i}-$ CSales $) /\left(\right.$ FSale $_{i}+$ CSale $), i=1,2,3,4,5$

First and Second Moments of the Subjective Growth Rate Distribution

Mean(SaleGr $)=\sum_{i=1}^{5} p_{i}$ SaleGr $_{i}$

$\operatorname{Var}($ SaleGr $)=\sum_{i=1}^{5} p_{i}\left(\right.$ SaleGr $_{i}-$ Mean(SaleGr $\left.)\right)^{2}$

$S D($ SaleGr $)=\sqrt{\text { Var(SaleGr })}$ 


\section{A.1.4 Capital Investment Rates}

\section{Respondent Data}

CCap $=$ firm's capital investment expenditures in the current quarter, as reported by the respondent

$\mathrm{FCap}_{i}=$ capital investment expenditures 4 quarters hence, $i=1,2,3,4,5$

$p_{i}=$ the associated probabilities, $i=1,2,3,4,5$

$K=$ our measure of the firm's capital stock

\section{Current Investment Rate}

CInvRate $=\operatorname{CCap} / K$, which we winsorize at the $1^{\text {st }}$ and $99^{\text {th }}$ percentiles

First and Second Moments of the Subjective Distribution for Future Capex:

$\operatorname{Mean}(F C a p)=\sum_{i=1}^{5} p_{i} F_{C a p}$

$\operatorname{Var}(F C a p)=\sum_{i=1}^{5} p_{i}\left(F_{C a p_{i}}-\operatorname{Mean}(F C a p)\right)^{2}$

$S D($ FCap $)=\sqrt{\operatorname{Var}(\text { FCap })}$

\section{Capital Investment Rates (cont.)}

First and Second Moments of the Distribution of Future Investment Rates:

Mean (InvRate) $=$ Mean $(F C a p) / K$

$S D$ (InvRate) $=S D$ (FCap) $/ K$

We also winsorize these first and second moments at the $1^{\text {st }}$ and $99^{\text {th }}$ percentiles 


\section{A.1.5 Measuring Capital Stocks}

- In September and October 2017 as well as February and March 2019 we included the following special question with the CC (Capex/Unit Costs) questionnaire:

Please provide an estimate of the book value of all property, plant, and equipment owned by your firm.

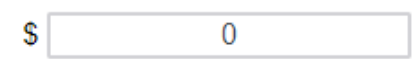

- We thus have data on our respondents' capital stock (PPENT) during at most two survey waves.

- Our goal is to approximate firm's actual investment rates $\left(\frac{I}{K}\right)_{t}$ in quarter $t$, as well as their expectations and uncertainty for future investment from the standpoint of quarter $t: \mathrm{E}_{\mathrm{t}}\left[\left(\frac{I}{K}\right)_{t+4}\right], \mathrm{SD}_{\mathrm{t}}\left[\left(\frac{I}{K}\right)_{t+4}\right]$ in all survey waves.

- We impute the firm's capital stock based on the responses to the special questions from September/October 2017 and February/March 2019 as follows:

- Case 1. We observe a firm's reported capital stock once:

In this case we impute the capital stock $K_{t}=K$, the reported capital stock for all survey waves $t$ the firm participates in.

- Case 2. We observe a firm's reported capital stock twice, once in 2017 and once in 2019: - In months prior to the first observation, we impute $K_{t}=K_{1}$, the first reported capital stock. - In months between the two observations, we impute $K_{t}=w_{t} * K_{1}+\left(1-w_{t}\right) * K_{2}$ where $w_{t}=\left(D_{2}-t\right) /\left(D_{2}-D_{1}\right), D_{i}, i=1,2$ is an integer representing the month in which we observe a reported capital stock, and $D_{1}<t<D_{2}$.

- Case 3. We do not observe the firm's reported capital stock in any survey wave: - We impute $K_{t}$ based on a regression $\log K_{f t}=\alpha_{s}+\alpha_{t}+\beta \log E_{f t}+\varepsilon_{f t}$ where $f$ indexes firms, $s$ indexes sectors, and $t$ indexes dates and $E=$ employment. Our estimate for $\hat{\beta}=$ $1.009(0.013)$ and the R-squared of the regression is 0.432 .

- After these imputations we have a (rough) measure of $K$ for most survey responses.

- We winsorize our measure of $K$ at the $1^{\text {st }}$ and $99^{\text {th }}$ percentile before starting the imputation procedure, and again before running the procedure in case 3 .

- Since May 2019, the core SBU questionnaire asks for the current value of the capital stock directly (See Figure A.1), so we no longer need to impute the value based on employment or special questions. 


\section{A.1.6 Obtaining Realizations and Forecast Errors}

- Consider a firm's subjective mean employment growth in month $t$, looking 12 months ahead (Mean(EGr)).

- We measure the firm's realized employment growth Realized $(E G r)$ as follows:

- We record its realized employment level in month $t+12, C E m p_{t+12}$.

- We record Realized $(E G r)=2 *\left(C E m p_{t+12}-C E m p_{t}\right) /\left(C E m p_{t+12}+C E m p_{t}\right)$.

- If $C E m p_{t+12}$ is missing, we use $C E m p_{t+11}$ and define Realized(EGr) $=2 *$ $\left(C E m p_{t+11}-C E m p_{t}\right) /\left(C E m p_{t+11}+C E m p_{t}\right)^{*} 12 / 11$.

- If $C E m p_{t+11}$ is also missing, we use $C E m p_{t+13}$ and record Realized $(E G r)=$ $2 *\left(C E m p_{t+11}-C E m p_{t}\right) /\left(C E m p_{t+11}+C E m p_{t}\right) * 12 / 13$.

- If $C E m p_{t+13}$ is also missing, we use the same formula with $C E m p_{t+10}$, or with $C E m p_{t+14}$ as a last resort.

- We record the firm's forecast error for employment growth looking 12 months ahead $=$ Mean $(E G r)-$ Realized $(E G r)$.

- Consider a firm's subjective mean sales growth in month $t$ of quarter $q$, looking 4 quarters ahead (Mean(SaleGr)).

- We measure the firm's realized sales growth, Realized(SaleGr), as follows:

- We record its current quarterly sales level reported in month $t+12$, CSale $_{t+12}$.

- We record Realized (SaleGr $)=2 *\left(\right.$ CSale $_{t+12}-$ CSale $\left._{t}\right) /\left(\right.$ CSale $_{t+12}-$ CSale $\left._{t}\right)$.

- If CSale $_{t+12}$ is missing, we proceed differently depending on whether $t$ is the first, second, or third month of the quarter.

- If $t$ is the first month of the quarter, we then try CSale $_{t+13}$ and CSale $_{t+14}$ in that order.

- If $t$ is the second month of the quarter, we then try CSale $t_{t+11}$ and $C S a l e_{t+13}$ in that order.

- If $t$ is the third month of the quarter, we then try CSale $_{t+11}$ and CSale $_{t+10}$ in that order.

- This procedure ensures that we use the level of quarterly sales reported in quarter $q+4$, though not necessarily in month $t+12$.

-We record the firm's forecast error for sales growth looking four quarters ahead $=$ Mean $($ SaleGr $)-$ Realized $($ SaleGr $)$ 
- Consider a firm's subjective mean investment rate looking four quarters ahead, as recorded in month $t$ of quarter $q$ (Mean(InvRate)).

- We measure the firm's realized investment rate in quarter $q+4$ Realized(InvRate) as follows:

- We record their current quarterly capital expenditures level reported in month $t+12$, CCapt+12.

- We record Realized (InvRate $)=C C a p_{t+12} / K_{t}$. Here we use $K_{t}$ rather than $K_{t+12}$ to focus on changes in investment rather than changes in (potentially mis-measured) capital stocks. This is symmetrical with how we construct expectations of future investment Mean(InvRate) in Appendix A.

- If $C C a p_{t+12}$ is missing, we proceed differently depending on whether $t$ is the first, second, and third month of the quarter.

- If $t$ is the first month of the quarter, we then try $\operatorname{CCap}_{t+13}$ and $C \mathrm{Cap}_{t+14}$ in that order.

- If $t$ is the second month of the quarter, we then try $\mathrm{CCap}_{t+11}$ and $\mathrm{CCap}_{t+13}$ in that order.

- If $t$ is the third month of the quarter, we then try $C \mathrm{Cap}_{t+11}$ and $C \mathrm{Cap} p_{t+10}$ in that order.

- This procedure ensures that we use the level of quarterly capital expenditures reported in quarter $q+4$, though possibly not in month $t+12$.

- We record the firm's forecast error for its investment rate looking four quarters ahead $=$ Mean $($ InvRate $)-$ Realized $($ InvRate $)$. 


\section{A.2 More Information about the SBU Recruitment Process and Panel}

The SBU's panel of respondents consists of firms from throughout the United States economy. With the exception of agriculture and government, our panel includes firms from every sector and a broad range of sizes (in terms of number of employees), from owner-operated firms to large publicly-traded companies.

\section{Panel Recruitment Process}

A team of research assistants at the Atlanta Fed identifies and recruits new panel members using lists of eligible firms purchased from an affiliate of Dunn \& Bradstreet, a supplier of business information and research. We requested that the lists include a proportion of firms in each (broad, one-digit) sector according to sectoral contribution to US Gross Domestic Product. We expect that the sampling universe in Dunn \& Bradstreet differs from the US Census' since small and young firms less likely to appear in the former data, whereas the Census observes the universe of establishments and firms with employment. The recruiting team deduplicates lists of contacts that we subsequently purchase, preventing us from re-recruiting previously listed firms.

The team of research assistants at the Atlanta Fed randomly selects potential recruits from a contact list, focusing on contacts in senior finance or executive roles. Since our goal is to use the survey to create indices that aggregate business expectations and uncertainty, the team oversamples firms with more than 100 and 500 employees. Figure A.5 uses a bin-scatter plot to show that the probability of being contacted increases with firm size. Our recruiters contact potential respondents via telephone, explaining the nature of the survey, its purpose, and informs them that individual survey responses are confidential. If the contact agrees to join the survey, the recruiter records his or her email address, where we deliver the personalized link to the survey 
instrument each month. We verify that the email address is valid by sending a confirmation that they have joined the SBU panel.

During the period covering June 2014 to June 2018, approximately 42 percent of potential contacts reached via telephone agreed to join the panel. Among those who joined, 62 percent responded at least once. In any given month about 43 percent of all continuing panel members responded to the survey. ${ }^{1}$ We believe these are fairly high and adequate response rates for a voluntary and complex survey.

To maintain the survey's sample size over time, we constantly recruit new firms to join the panel and replace those who stop responding. Our aim is to maintain a sample size of about 300 or more responses per month.

Figure A.6 shows how the equally-weighted and employment-weighted firm distribution in the Census Bureau's 2015 Statistics on US Businesses compares with: (1) our sampling frame; (2) the sample of firms we contact; and (3) those who ultimately respond. We report the comparison separately in terms of firms size, industry sector, and region. Focusing on firm size in Figure A.6a, the sampling frame indeed under-samples small firms (with less than 20 employees), and over-samples medium to large firms (with more than 20 and less than 500 employees). As we saw in Figure A.5, our recruiters contact larger firms with a higher probability, in particular those with more than 100 employees. Finally, looking at the employment-weighted distribution of SBU responses, we see that it is skewed towards larger firms. This finding is a robust fact of voluntary firm level surveys. For example, the Decision Maker Panel survey fielded by the University of Nottingham in collaboration with the Bank of England, which uses the SBU's methodology to

\footnotetext{
${ }^{1}$ These response rates refer to the period between September 2016 (when we made the most recent major change to the survey) and October 2018.
} 
elicit five-point subjective probability distributions, has a similar skew towards larger firms (see Bloom et al., 2018).

Figure A.7 repeats the comparison between the unweighted and employment-weighted composition of our survey panel against the rest of the US economy in terms of firm size, sector, age, region. Figure A.7e also shows the share of firms and share of employment in our sample belonging to publicly traded firms, as self-reported answers to special questions fielded in February and March 2019. Figures A.6 and A.7 also appear in the Online Appendix of Barrero (2019), which also uses SBU data. Figure A.8 shows the distribution of respondents (one per firm) according to their job titles. CFOs and other financial managers account for about 60 percent of respondents, with other C-level managers accounting for about 20 percent, and owners for about 10 percent. Thus, our respondents are primarily business executives who should actively participate in budgeting, forecasting, and decision making.

Table A.1 asks whether we can predict continued participation in the survey based on observable firm characteristics. As we already knew from Figure A.6a, larger firms are more likely to responding to a subsequent survey. We find that the magnitude of expectations and uncertainty do not correlate strongly with subsequent participation, which eases worries that our sample of loyal respondents may differentially select firms that are relatively optimistic or pessimistic, or more or less uncertain. While fixed effects for time, sector, region, or firm increase the R-squared of these predictive regressions, the within R-squared is small and the same order of magnitude as the R-squared from regressions that do not include fixed effects.

\section{Data Collection, Preparation, and Cleaning}

In a typical month, we email our respondents an individual link to the survey instrument on the Monday of the second full week of the month. We collect responses during the next two 
workweeks, so data collection ends on the Friday of the third full week of the month. The Monday following the end of the survey collection, we download and store all responses in a folder that contains all prior monthly data files. Then we run programs to combine all monthly files into an aggregate file and perform a series of automated cleaning procedures on the raw survey data. This cleaning program includes the following processes:

1. Rescaling of subjective probabilities: On occasion, respondents provide subjective probabilities that do not add up to 100 percent. We rescale probability vectors that add to between 95 and 105 percent to make them add up to 100 percent. We disregard responses whose probability vectors add up to a number outside the 95-105 percent range. Typically, this filter eliminates very few of the responses in a given month.

2. Adjustment of estimates and probabilities given in reverse order: In rare instances, some respondents provide their range of estimates in reverse order, starting with their "highest case" value in the "lowest case" scenario. We reverse these estimates and their associated probabilities to conform to the typical response pattern of lowest to highest.

Once the automated cleaning processes are completed, we perform a manual review of all large firms (firms with 1000 employees or more). We check a large firm's current month responses for consistency with its historical responses. If responses are found to be inconsistent, we conduct a review of publicly available information, including news reports, public filings, etc. If a review of publicly available information is inconclusive, we consider contacting the respondent for clarification. We focus on larger firms for the manual audit because of their greater weight on the aggregate indices produced from the SBU survey.

We also conduct a manual review of forecast errors once a month, as we describe in the main text. After computing realized growth rates (for employment in the 12 months after a survey, 
for sales in the four quarters following a survey), we compute the forecast error for a firm responding in month $t$ as the difference between the ex-ante subjective mean and the realized growth rate we record in the data. We manually review the responses of firms whose forecast errors for employment and sales growth exceed one in absolute value. We use the firm's history of responses about current sales and employment to correct obvious mistakes. Common mistakes include missing or added zeros and reporting an annual rather than a quarterly sales figure. If we cannot find an obvious mistake, we flag these observations as potential errors, typically excluding them from analyses of forecast error behavior. 


\section{Figure A.4: Time Series of the Number of SBU Responses}
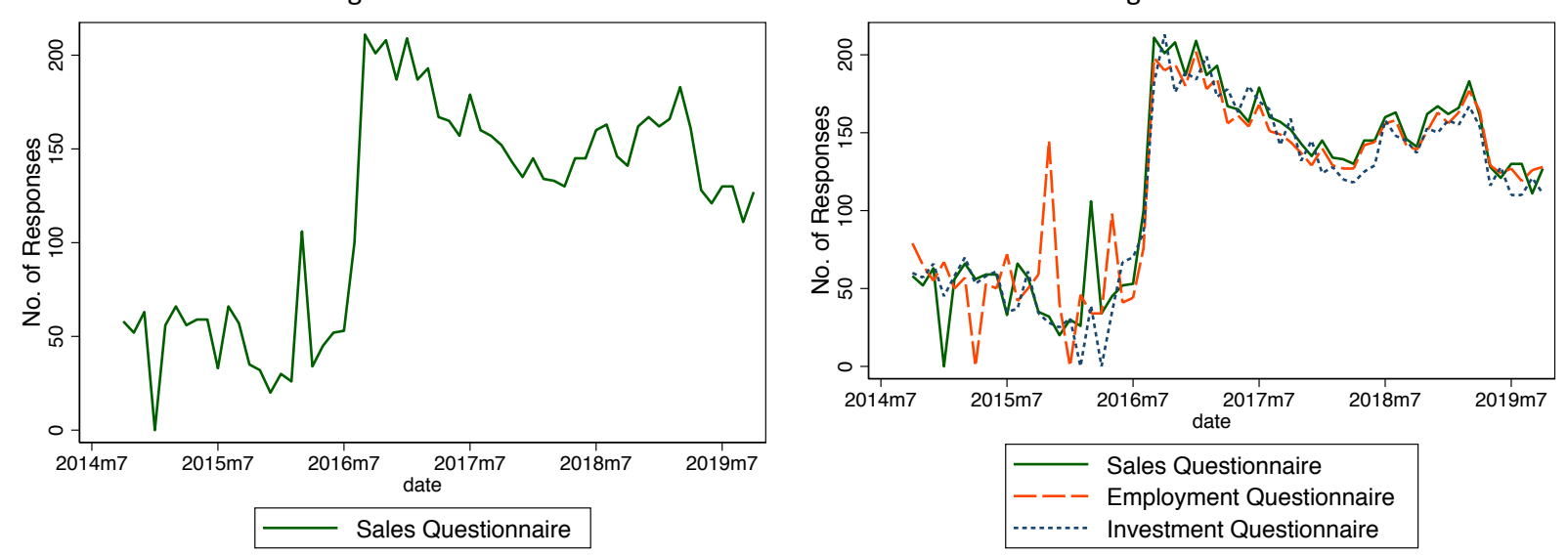

Notes: The figure on the left shows the number of responses to the SBU's sales questionnaire for which we can compute an expectation for sales growth over the next four quarters. The figure on the right shows the number of responses to the sales, employment, and investment questionnaires for which we can compute, respectively, expected sales growth for the next four quarters, employment growth for the next twelve months, and the expected investment rate four quarters ahead. Data are from the SBU and include all survey months between 10/2014 and 10/2019.

\section{Figure A.5: Probability of being contacted by our research team as a function of firm size}

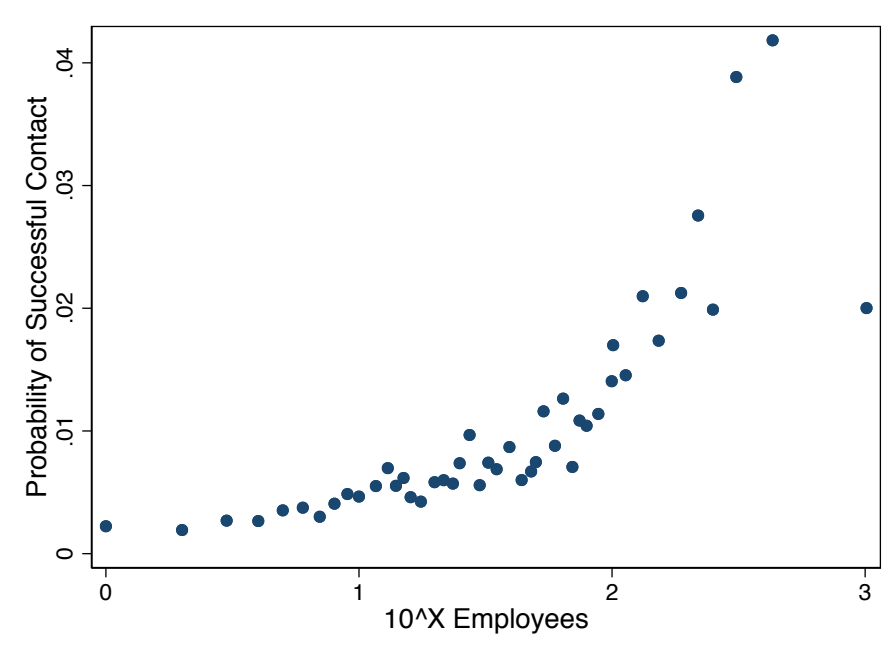

Notes: This figure shows the probability with which the team of SBU recruiters successfully contacts a firm in the SBU's sampling frame, for each percentile of the firm size distribution in the sampling frame (on the horizontal axis). We say that a firm is successfully contacted if our recruiting team speaks to a person at the firm in question. Data are from the SBU's sampling frame combined with the recruiting team's call log data as of October 2018. 


\section{Figure A.6: The SBU sampling frame and sample A.6a. Firm Size}

Figure A.6a.i

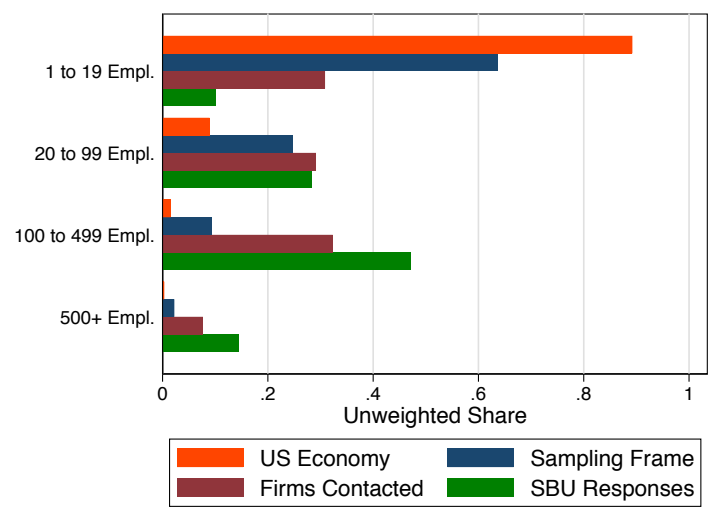

Figure A.6b.ii

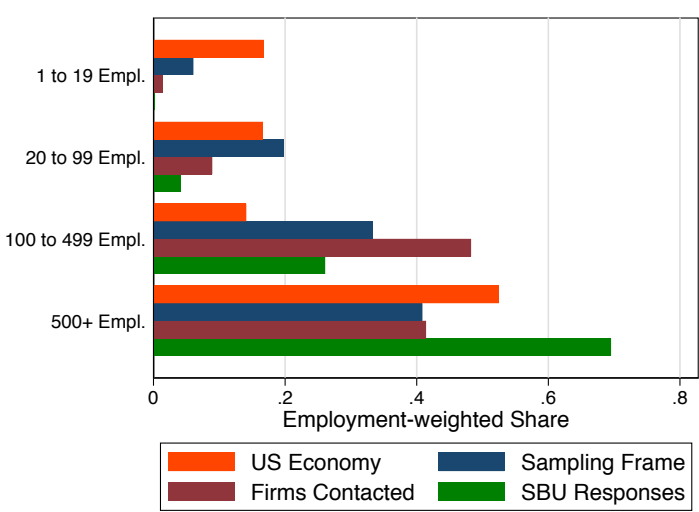

Notes: The above figures show the unweighted (left) and employment-weighted (right) shares of (1) firms in the US economy, (2) the SBU's Dunn \& Bradstreet sampling frame, (3) firm that SBU recruiters contact successfully, and (4) SBU responses across each of the five employment categories shown on the vertical axis. The SBU data covers all waves of the SBU from 10/2014 to 10/2019. Data for the US Economy come from the US Census Bureau's 2015 Statistics on US Businesses. An observation in the SBU is a response for which we can construct a subjective probability distribution for one of employment, sales, investment, or unit cost growth looking one year ahead. Data for the sampling frame include all purchased lists and call logs up to October 2018. We say that SBU recruiters contact a firm successfully if they manage to speak to an individual at the firm.

\section{A.6b. Industry}

Figure A.6b.i

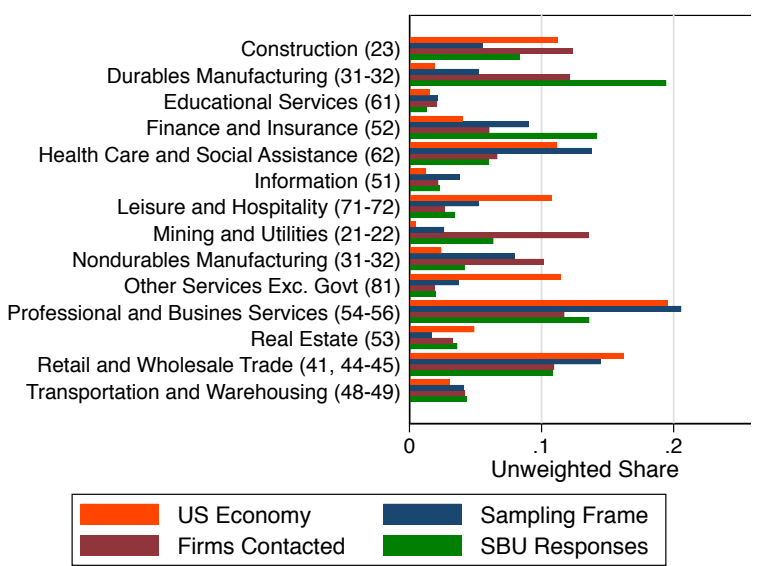

Figure A.6b.ii

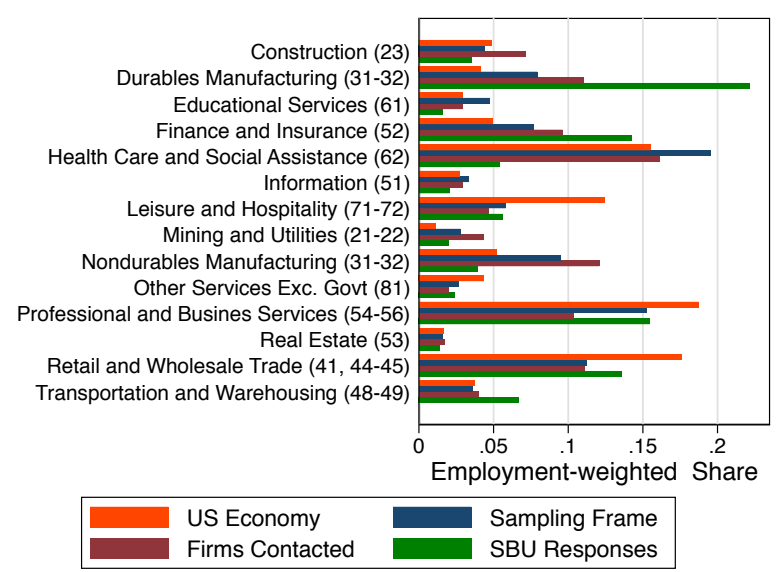

Notes: The above figures show the unweighted (left) and employment-weighted (right) shares of (1) firms in the US economy, (2) the SBU's Dunn \& Bradstreet sampling frame, (3) firm that SBU recruiters contact successfully, and (4) SBU responses across each of the industry sectors shown on the vertical axis. The SBU data covers all waves of the SBU from 10/2014 to 10/2019. Data for the US Economy come from the US Census Bureau's 2015 Statistics on US Businesses. An observation in the SBU is a response for which we can construct a subjective probability distribution for one of employment, sales, investment, or unit cost growth looking one year ahead. Data for the sampling frame include all purchased lists and call logs up to October 2018. We say that SBU recruiters contact a firm successfully if they manage to speak to an individual at the firm. 


\section{A.6c. Region}

Figure A.6c.i

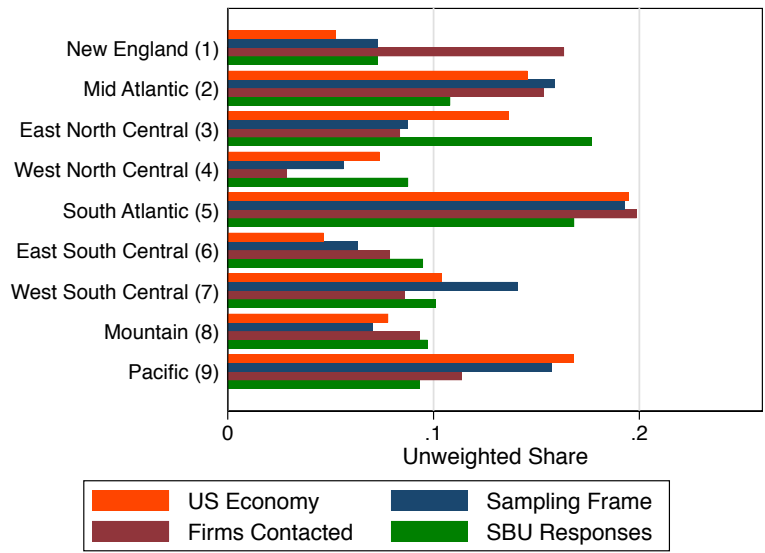

Figure A.6c.ii

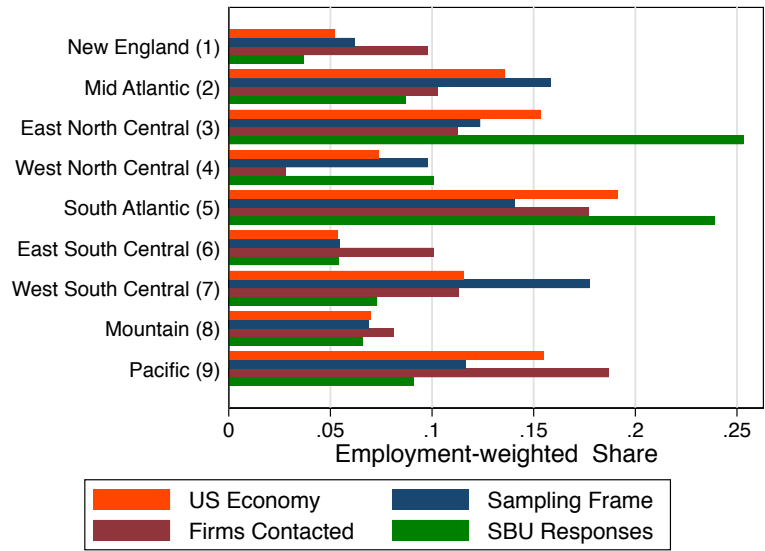

Notes: The above figures show the unweighted (left) and employment-weighted (right) shares of (1) firms in the US economy, (2) the SBU's Dunn \& Bradstreet sampling frame, (3) firm that SBU recruiters contact successfully, and (4) SBU responses across each of the regions (Census Divisions) shown on the vertical axis. The SBU data covers all waves of the SBU from 10/2014 to 10/2019. Data for the US Economy come from the US Census Bureau's 2015 Statistics on US Businesses. An observation in the SBU is a response for which we can construct a subjective probability distribution for one of employment, sales, investment, or unit cost growth looking one year ahead. Data for the sampling frame include all purchased lists and call logs up to October 2018. We say that SBU recruiters contact a firm successfully if they manage to speak to an individual at the firm.

\section{Figure A.7: SBU Panel Distributions vs US Economy A.7a. Firm Size}
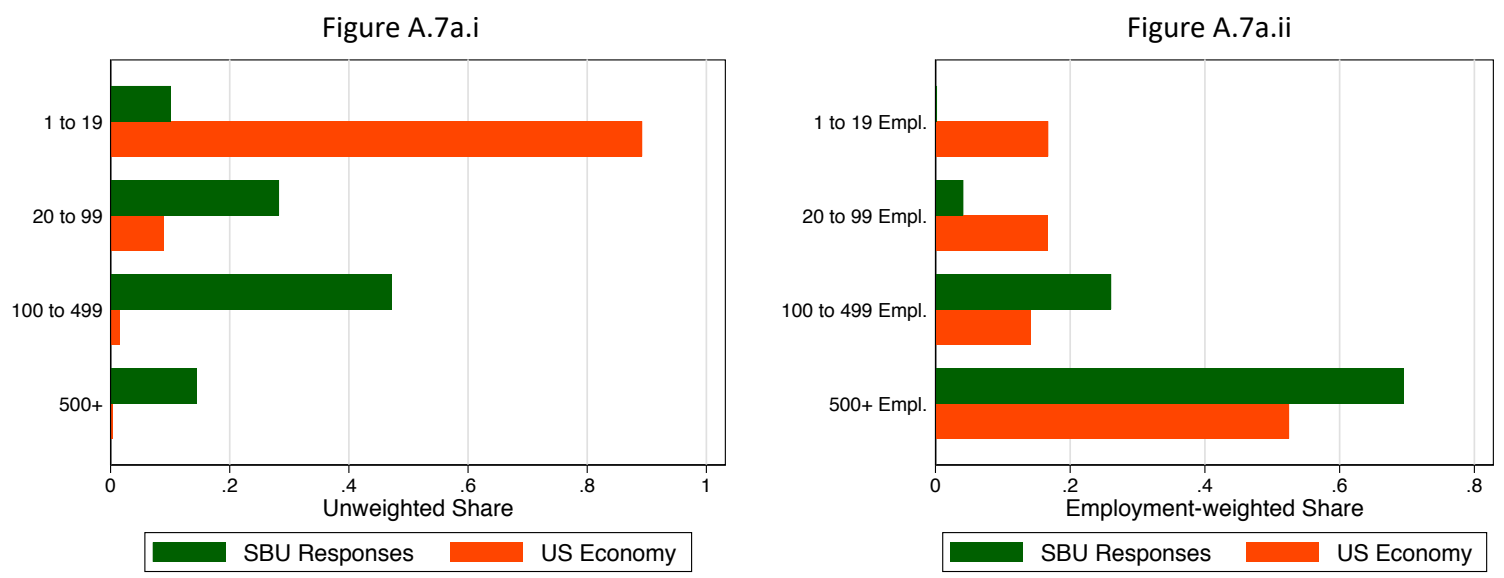

Notes: The above figures show the unweighted (left) and employment-weighted (right) shares of (1) SBU responses and (2) the US Economy accounted for by firms each of the five employment categories shown on the vertical axis. The SBU data covers all waves of the SBU from 10/2014 to 10/2019. Data for the US Economy come from the US Census Bureau's 2015 Statistics on US Businesses. An observation in the SBU is a response for which we can construct a subjective probability distribution for one of employment, sales, investment, or unit cost growth looking one year ahead. 


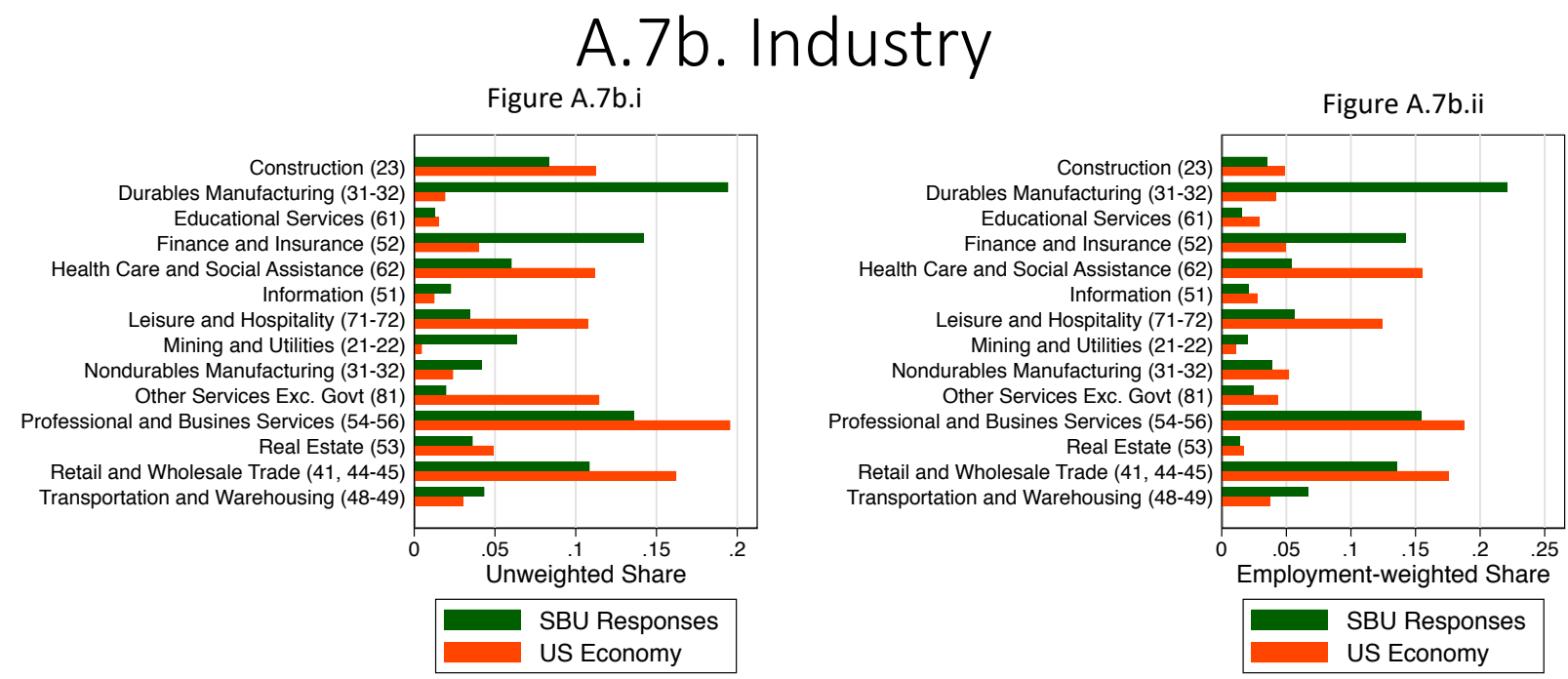

Notes: The above figures show the unweighted (left) and employment-weighted (right) shares of (1) SBU responses and (2) the US Economy accounted for by firms each of the sectors shown on the vertical axis. The SBU data covers all waves of the SBU from 10/2014 to 10/2019. Data for the US Economy come from the US Census Bureau's 2015 Statistics on US Businesses. An observation in the SBU is a response for which we can construct a subjective probability distribution for one of employment, sales, investment, or unit cost growth looking one year ahead.

\section{A.7c. Geography}

Figure A.7c.i

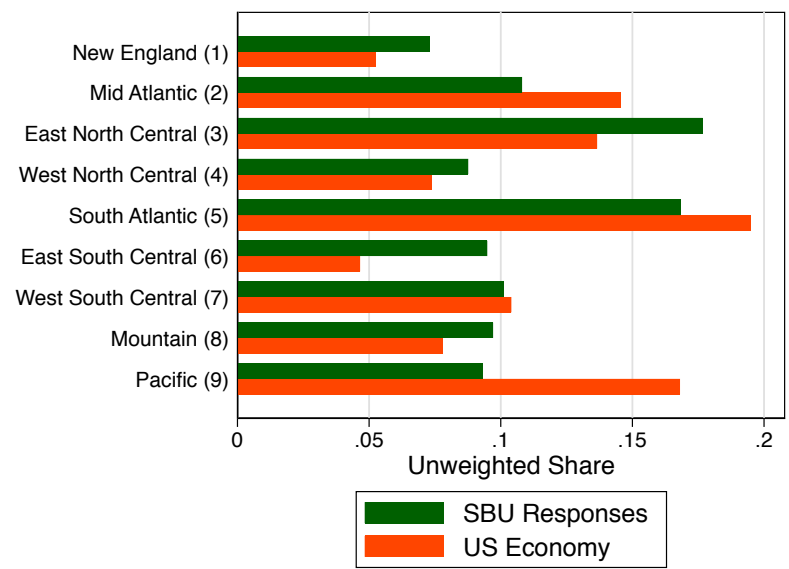

Figure A.7c.ii

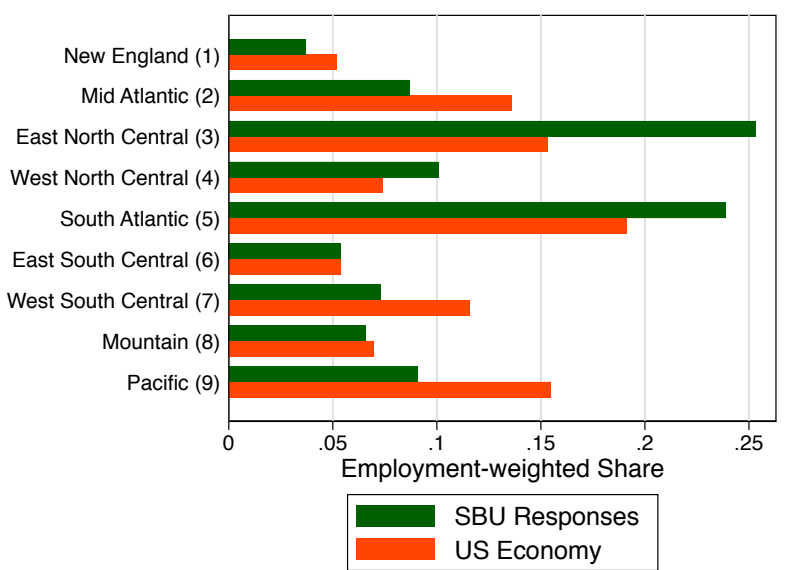

Notes: The above figures show the unweighted (left) and employment-weighted (right) shares of (1) SBU responses and (2) the US Economy accounted for by firms in each Census Division as shown on the vertical axis. The SBU data covers all waves of the SBU from 10/2014 to 10/2019. Data for the US Economy come from the US Census Bureau's 2015 Statistics on US Businesses. An observation in the SBU is a response for which we can construct a subjective probability distribution for one of employment, sales, investment, or unit cost growth looking one year ahead. 


\section{A.7d. Firm Age}

Figure A.7d.i

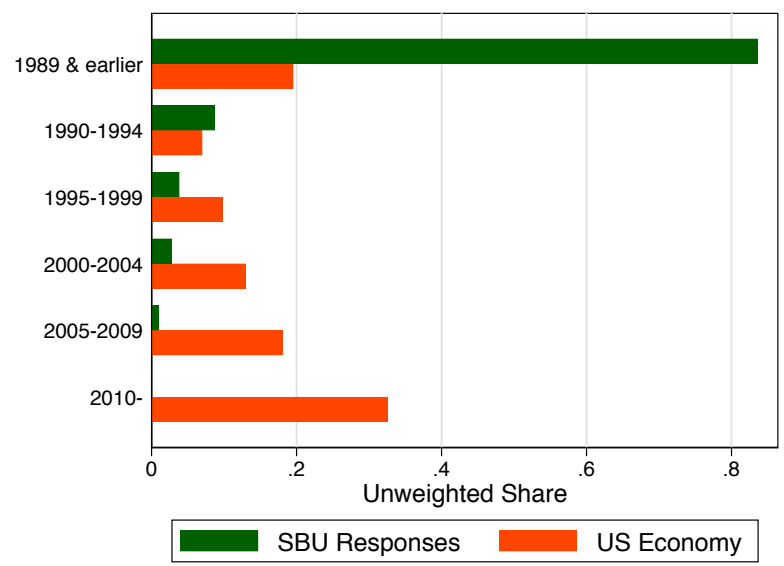

Figure A.7d.ii

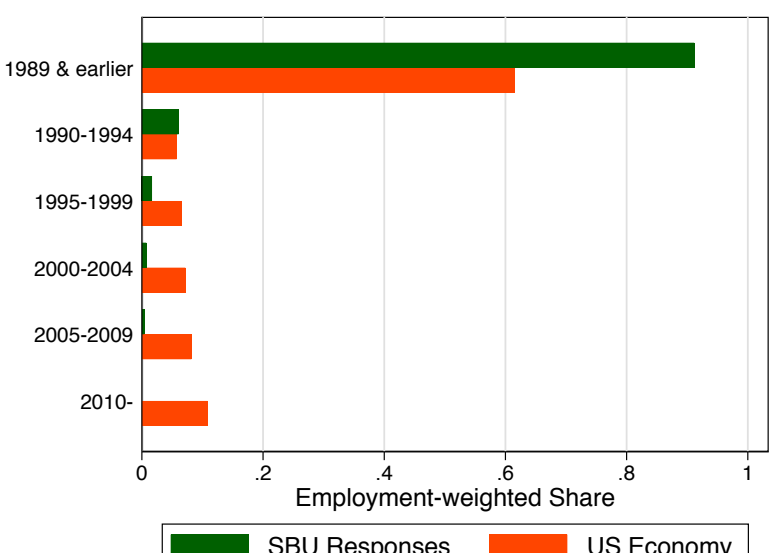

Notes: The above figures show the unweighted (left) and employment-weighted (right) shares of (1) SBU responses and (2) the US Economy accounted for by firms who hired their first paid employee during the years listed on the vertical axis. The SBU data covers all waves of the SBU from 10/2014 to 10/2019. Data for the US Economy come from the US Census Bureau's 2015 Business Dynamics Statistics. An observation in the SBU is a response for which we can construct a subjective probability distribution for one of employment, sales, investment, or unit cost growth looking one year ahead. We obtained information on when SBU respondents hired their first paid employee based on a special question that accompanied the core SBU survey in January 2017.

\section{A.7e. Publicly-traded vs. Privately-held}

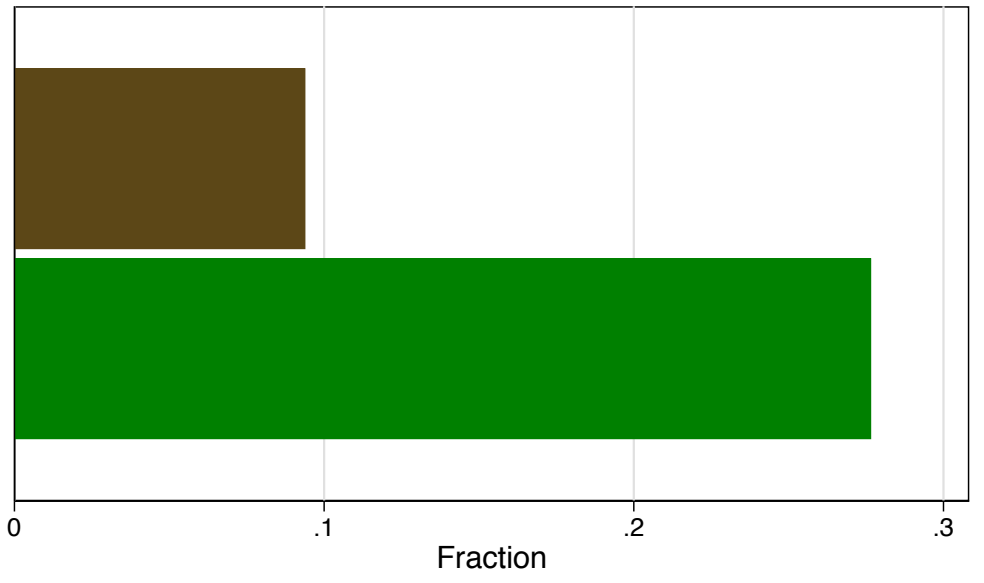

Publicly-traded Firms Share

Employment Share Publicly-traded Firms
Notes: This figure shows the share of unique firms in the SBU and the share of employment among all SBU responses accounted for firms whose shares traded in a stock exchange or over-thecounter markets.*

*We determine whether a firm is publiclytraded based on a special survey question from February and March 2019 


\section{Figure A.8: Job Titles of SBU Respondents}

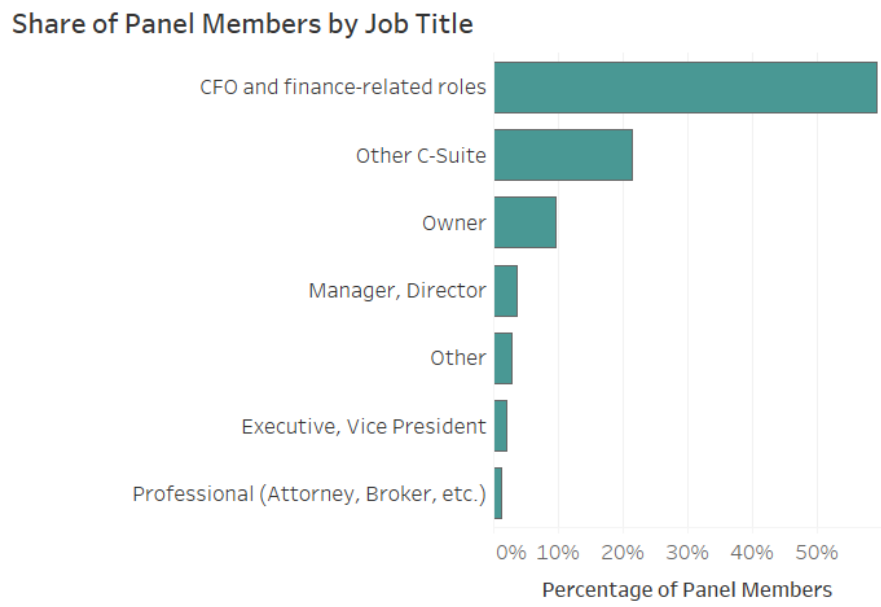

Notes: This figure shows the percentage of SBU panel members whose job title corresponds to the categories on the vertical axis. The sample includes all firms that have been part of the SBU panel at any point between October 2014 and January 2020. The unit of observation is a firm.

\section{Table A.1: What variables predict continued participation in the survey?}

\begin{tabular}{|c|c|c|c|c|c|c|c|c|}
\hline \multirow{3}{*}{$\begin{array}{l}\text { Dependent Variable } \\
\text { Estimator }\end{array}$} & (1) & (2) & (3) & (4) & (5) & (6) & (7) & (8) \\
\hline & \multicolumn{8}{|c|}{ 1(Firm Responds to a Subsequent Survey) } \\
\hline & OLS & OLS & OLS & OLS & OLS & OLS & OLS & Probit \\
\hline $\log ($ Employment $)$ & $\begin{array}{c}0.017 * * * \\
(0.005)\end{array}$ & & & & $\begin{array}{l}0.008 * \\
(0.004)\end{array}$ & $\begin{array}{c}0.000 \\
(0.005)\end{array}$ & $\begin{array}{c}0.005 \\
(0.021)\end{array}$ & $\begin{array}{l}0.031^{*} \\
(0.016)\end{array}$ \\
\hline Expected Sales Growth, Next 4 Quarters & & $\begin{array}{l}-0.012 \\
(0.077)\end{array}$ & & $\begin{array}{l}-0.008 \\
(0.077)\end{array}$ & $\begin{array}{l}-0.008 \\
(0.077)\end{array}$ & $\begin{array}{c}0.001 \\
(0.078)\end{array}$ & $\begin{array}{c}0.021 \\
(0.071)\end{array}$ & $\begin{array}{l}-0.036 \\
(0.283)\end{array}$ \\
\hline $\log$ (Sales Growth Uncertainty), Next 4 Quarters & & & $\begin{array}{l}-0.009 \\
(0.007)\end{array}$ & $\begin{array}{l}-0.009 \\
(0.007)\end{array}$ & $\begin{array}{c}0.010 \\
(0.007)\end{array}$ & $\begin{array}{c}0.002 \\
(0.009)\end{array}$ & $\begin{array}{c}-0.018^{* *} \\
(0.009)\end{array}$ & $\begin{array}{c}0.035 \\
(0.026)\end{array}$ \\
\hline Industry FE & & & & & & $\mathrm{Y}$ & & \\
\hline Region FE & & & & & & $\mathrm{Y}$ & & \\
\hline Time FE & & & & & & $\mathrm{Y}$ & $\mathrm{Y}$ & \\
\hline Firm FE & & & & & & & $\mathrm{Y}$ & \\
\hline Mean of Dep. Variable & 0.768 & 0.791 & 0.791 & 0.791 & 0.807 & 0.82 & 0.824 & 0.807 \\
\hline Observations & 17,388 & 7,159 & 7,156 & 7,153 & 6,873 & 6,275 & 6,612 & 6,873 \\
\hline (Pseudo) R-squared & 0.004 & 0.000 & 0.000 & 0.000 & 0.001 & 0.163 & 0.517 & 0.001 \\
\hline Within R-squared & $\overline{=}$ & 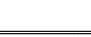 & = & 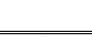 & = & $2.23 \mathrm{e}-05$ & 0.00102 & $=$ \\
\hline
\end{tabular}




\section{B. Additional Information about Survey Development and Testing}

We document the process through which we came up with the methodology to elicit fivepoint discrete subjective probability distributions from business executives. Table B.1 summarizes a series of question designs that we fielded and evaluated, initially as part of the BIE's special question series and later in a new panel of firms for the Survey of Business Uncertainty.

October 2013: Initial question formulations

We began fielding trial questions in October 2013, comparing two designs for eliciting information about the firm's subjective distribution over its future sales growth rate. Figure B.1 displays screen shots. We randomly assigned each question design to half the participants.

- The first design asked respondents to select the best, middle and worst-case percentage changes in the firm's sales over the next twelve months. A drop-down menu for each case let respondents choose among values ranging from -10 to +25 in one-point increments. Pop-up boxes instructed respondents to select a "best case" corresponding to the top ten percent of possible outcomes, a "worst case" corresponding to the bottom ten percent, and a "middle case" corresponding to a value the firm would use for planning purposes.

- The second design asked respondents to assign probabilities to five pre-set interval bins for the possible percentage change in sales over the next year. The bins ranged from "less than -1 percent" at the bottom end to "more than 5 percent" at the top end.

The first design resembles that of the Duke CFO Survey question about future stock market returns, and the second is closer to that of the Bank of Italy's Survey on Investment in Manufacturing. These two long-running surveys of business mangers offered a natural starting point for thinking about SBU question design. We were particularly interested in two issues: First, 
whether the two designs yield similar inferences about mean expectations and uncertainty, and second, the adequacy of the intervals in the five-bin design.

Using the October 2013 responses, we constructed subjective distributions and compared four moment statistics. ${ }^{2}$ The first design yielded a higher mean expectation than the second design (4.2 and 1.9 percent, respectively, for the expected sales growth rate), greater dispersion in expected sales growth rates (standard deviations of 5.9 and 1.7 percent), higher subjective uncertainty (average standard deviation values of 3.6 and 1.4 percent), and more dispersion in subjective uncertainty (standard deviations of 2.1 and 0.8 percent). For each moment, we reject the null hypothesis of equality across the two question designs at a p-value under 0.001. Clearly, the two question designs yield quite different inferences about firm-level forecast distributions.

Each question design also has potentially serious weaknesses for our purposes. The first design allows for only three support points, which affords a rather coarse characterization of the subjective probability distribution. Moreover, the pre-set outcome range in the drop-down menu may inject anchoring effects that distort the responses. Regarding the second design, a large body of literature shows that (a) businesses differ greatly in their realized growth rates and (b) much of the mass in the realized growth rate distribution lies outside the lowest and highest values $(-1$ percent and 5 percent) specified in the question. See, for example, the literature review in Davis and Haltiwanger (1999). Taken together, (a) and (b) imply that it is infeasible to pre-specify a modest number of support points or bins that allow all firms to characterize their subjective forecast distributions in a reasonably granular manner. This observation argues strongly in favor of letting respondents select the support points. Our survey responses to the second question design

\footnotetext{
${ }^{2}$ The first design yields a three-point discrete distribution with probability 0.1 for the "worst" case, 0.8 for the "middle" case and 0.1 for the "best." For the second design, we applied the user-selected probability to the interval midpoint. We used $6 \%$ and $-2 \%$ for the top and bottom intervals, respectively.
} 
suggest another reason as well. In particular, the typical respondent assigned a probability of about 30 or 40 percent to the middle bin (1.1 to 3 percent sales growth) and 10 to 20 percent to the outer bins. This pattern is worrisome in light of empirical regularities (a) and (b). It suggests that the question design leads respondents to put too much mass in our pre-set middle bin. This response pattern fits the "middle means typical" heuristic, a well-known source of response distortion in the survey design literature (e.g., Tourangeau, Couper, and Conrad, 2004).

November 2013: Alternative interval bins

We retained the first question design in November 2013 but made it clearer that the worst, middle and best cases correspond to support points with pre-set probabilities of 0.1, 0.8 and 0.1. We tried a variant of the second design with much wider interval bins. Figure B.2 shows both of the questions we tried in November. The wider bins more closely align with the range of outcomes elicited by the first question design in October 2013 and better reflect the heterogeneity in observed firm-level growth rates.

The November 2013 results showed that the spread of the bin intervals in the second design matter greatly. In particular, the moment statistics generated by the second question design in November 2013 are much closer to the ones generated by the first design in either month. For example, the second design yielded an average expected growth rate of 5.1 percent and average uncertainty statistic of 5.8 percent in the November survey. Moreover, the same firms responded quite differently to the October and November variants of the second question design. Among firms that received and answered the second question in both months, the average expected growth rate jumped from 1.7 to 5.1 percent, and the average subjective standard deviation jumped from 1.1 to 4.5 percent. In sharp contrast, among the firms that received and answered the first question in both months, the average expected growth rate was nearly identical ( 3.3 and 3.2 percent), and 
the average subjective standard deviation was similar at 3.4 percent in October and 3.0 percent in November. Finally, among firms that got the second design in October and the first design in November, the moment statistics differ between months very similarly to how they differ across the two designs in October.

The foregoing discussion underscores two advantages of letting respondents select the location of support points. First, it allows for a parsimonious question format (as in the first design) while still accommodating enormous cross-firm heterogeneity in the central tendency and dispersion of growth rates. Second, it avoids anchoring and types of response distortions that might be introduced by pre-specifying support points or interval bins.

December 2013: Testing three-point versus five-bin designs on unit cost questions

In December 2013, we modified the questions to refer to unit cost growth over the next 12 months. Figure B.3 shows screen shots. Again, the two question designs yielded systematic differences in the moment statistics. Although the between-design discrepancies in December 2013 for unit cost growth were smaller than the ones for sales growth in October 2013, the results reinforced our concerns about the pitfalls in pre-specifying the support points or bins.

January 2014: Freeing up the probabilities

In January 2014, we began testing designs that let respondents freely select probabilities and support points. We used a three-point distribution and returned to sales growth. We modified the questionnaire to refer to scenarios for "low", "medium", and "high" growth, instead of the "worst," "most likely," and "best" scenarios. Figure B.4 shows screenshots.

With this design, respondents reported statistically significantly higher subjective uncertainty and - particularly — greater heterogeneity in both forecasts and subjective uncertainty concerning sales growth over the next 12 months. Letting respondents provide their own 
probabilities typically yielded more weight on the "high" and "low" scenarios - closer to 20 or 25 percent than the 10 percent specified in the October and November variants of this question. Respondents also assigned a broad range of probabilities to "high" and "low" scenarios, typically from 5 to 30 percent but in extreme cases as low as zero or as high as 80 or 85 percent. By contrast, the three support point values they selected were similar to the ones they gave in October and November. We did not remind respondents that probabilities should add up to 100 percent. Indeed, 20 percent submitted probability vectors that did not add up to 100 .

The January 2014 experiment led us to conclude that letting respondents select support points and probabilities is feasible and allows them to express idiosyncratic features of their subjective probability distributions. From a research standpoint, this question design means our survey questions can capture heterogeneity in expectations and uncertainty in the cross-section of firms as well as within firms over time.

\section{February 2014: Testing a question about employment}

In February 2014 we essentially replicated the experiment from January, but now asked BIE respondents to provide a three-point subjective probability distribution for their firm's employment 12 months in the future. We first asked them for the firm's current number of employees (including part-time). Then we asked them to provide three-point outcomes for the level of employment ("low", "middle", and "high") twelve months into the future, and then assign a probability to each of those three outcomes. See Figure B.5 for a screenshot.

The February test showed BIE respondents were willing and able to express a three-point discrete distribution for future employment levels. Similar to our prior tests of questions about sales growth and unit cost growth, the employment levels question had a low rate of item nonresponse, respondents gave monotonic outcomes across the "low", "medium", and "high" 
cases, and 98 percent of their probability assignments summed to 100 percent. Thus, we found it feasible to obtain expectations and uncertainty about employment, which is a key indicator of firm size and performance in the firm dynamics literature (e.g. see Davis and Haltiwanger, 1999).

March 2014: Repeating the three-point, three-probability sales question from January 2014

The March 2014 BIE special question included a repeat of the January 2014 question, namely asking respondents for "low", "middle", and "high" scenarios for sales growth over the next year and subsequently ask them to assign a probability to each of these scenarios. (See Figure B.6 for a screenshot.) We confirmed the suitability of the question and found responses to be broadly consistent with those from January.

April-May 2014: Testing five-point, five-probability versions of the sales and employment questions

We decided to test a five-point version of the sales question in April 2014 (again as a special question in the BIE survey), to see whether and how respondents took up the added flexibility. We were also interested to see if we could capture more extreme outcomes by asking for two additional "highest" and a "lowest" scenarios at the tails of the elicited sales growth distribution. (Figure B.7 shows a screenshot of this version of the question.) We found this test to be successful, with many respondents assigning more extreme outcomes and lower probabilitieson the order of 10 percent - to the extreme outcomes.

In May 2014 we tested a five-point version of the employment question from February 2014. The screenshot for this test, again implemented among BIE respondents, is shown in Figure B.8. Once more, we found this test to be successful, with respondents assigning low probabilities to the outermost "highest" and "lowest" scenarios and associating those outcomes with more extreme outcomes. 
June 2014: Testing a three-point version of the sales question, plus asking for extreme scenarios

In June 2014 we returned to the three-point sales question from January and March and considered how responses changed if we asked for "best" and "worst" tail scenarios in addition to the three-point distribution, without asking for associated probabilities for those tail scenarios. Figure B.9 shows a screenshot of this test.

Responses to the three-point question in this experiment yielded forecasts and subjective uncertainty over future sales that had a similar mean and dispersion as those from January and March. We interpreted this consistency between January, March, and June responses as a sign of the reliability of our methodology.

June 2014 was the last time we tested questions as part of the BIE special question series. July 2014: First tests on the new SBU panel

From July 2014 on, we conducted survey testing on a new panel of firms recruited specifically for the Survey of Business Uncertainty (described in more detail in Online Appendix E below). Following the $\mathrm{A} / \mathrm{B}$ testing strategy employed previously, we split the sample randomly and sent three-point or five-point versions of the questions, now covering three topics: employment, sales growth, and prices. See Figure B.10 for screenshots of these questions.

In the inaugural SBU survey, we found that respondents were willing and able to provide monotonic scenarios (i.e. the "lowest" support point is less than the "low" support point, etc.) across the five support points for the outcomes, that probability vectors nearly always summed up to 100 percent, and that the distributions of respondents' implied subjective expectations and uncertainty resembled those of the three-point questions we tested in previous months. Additionally, as we first found in the April 2014 test, the five-point questions gave respondents additional flexibility to express their perception of outcomes farther out on the tails. As a 
consequence of these findings we decided to focus on our five-point question design going forward.

$\underline{\text { Summer and Early Fall 2014: Cognitive interviews and further development of questions }}$

During summer 2014, the team conducted cognitive interviews with 7 members of the BIE panel to assess their understanding of the questions that constituted the new SBU survey. Most interviewees found the questions to be interesting, worthwhile, and user-friendly. Much of the feedback they provided was quite industry-specific and thus not particularly actionable since we wanted our survey to work for firms throughout the private business sector.

One useful finding from the interviews concerned the way we were asking respondents to select the five potential support point outcomes ("lowest" to "highest"). Up to that point, we had been using drop-down boxes with one-unit increments. For example, the bottom box would correspond to -20 percent (or lower) sales growth over the next year, the next to -19 , the following one -18 percent, and so forth. Many respondents asked the increments to be finer in order to increase precision.

Additionally, we found that the drop-down boxes could be problematic. This was especially true for sales, where we had made the range covered the drop-down box very large (from less than -24 percent in the lowest case to more than +35 percent in the highest). Some respondents confused the minus with a dash and thus ended up selecting the wrong outcome. Others had difficulty working with such a large drop down menu. In light of these comments and observations, we moved to an open-text question format, allowing respondents to enter the values each of the support point outcomes freely for all questions. We tested that question design in August 2014, which was the same as in July except for the replacement of the drop-down boxes with open text boxes. 
In early fall 2014 we also changed the format of the sales questionnaire to mimic that of employment, namely asking for the current level of sales in dollars and asking for five potential sales levels one year ahead, using an open-text format. We had been using this open-text, free selection approach for the questions on employment levels because it was harder for us to preset the support point outcomes in the presence of vast heterogeneity in the number of employees across firms. We additionally changed the wording in the sales question to refer to quarterly values, given that sales are a flow rather than a stock variable and are often tracked quarterly. See Figure A.2, for a look at the revised sales question.

During the August test we also tested questions on unit costs (which we had previously tested in December 2013), capital investment (following the new sales question, see Figure A.1) and new questions about profit margins (see Figure B.11). In September we conducted tests that were very similar to those of August, also trying out questions on average prices. ${ }^{3}$

\section{October 2014: Initial version of the SBU operates regularly}

In October 2014 we settled on the first stable version of the SBU questionnaire (at the time known as the "Decision Maker Survey"). Since then, the survey has been administered monthly out of the Atlanta Fed with monthly response rates averaging roughly 40 percent, resulting in about 300 responses per month. Up until October 2015 we divided the panel into three subgroups, each answering questions about two topics in a given month, with topics including employment, sales, capex, unit costs, average prices, and profit margins. From November 2015 to August 2016 we used six sub-groups, each answering questions about two of the six topics.

September 2016 to April 2019

\footnotetext{
${ }^{3}$ See Figure B.11 for the wording of the prices and profit margins questions. In subsequent rounds of the survey we eliminated the questions on these two topics and they are not part of our main analyses.
} 
We made a major change to the SBU in September 2016, at which time we eliminated the questions on average prices and profit margins. Based on feedback from our respondents these were the questions that created the most confusion. Eliminating these two topics also allowed us to split the sample into just two groups, greatly increasing the number of responses per topic per month. Starting in September 2016, the monthly SBU form thus contained two of the four topics in the following combinations: Average Unit Cost/Capital Expenditures (CC), and Sales Revenue/Number of Employees (SE). We sorted our panel respondents randomly into two subgroups. In a given month group A received the Sales Revenue/Number of Employees (SE) questionnaire and vice versa for group B.

In September 2016 we also changed the sales question back to asking about sales growth rates looking ahead over the next four quarters, rather than sales levels four quarters ahead. Figure A.2 reflects this change, in contrast with Figure $1 \mathrm{~b}$ in the main text. Our rationale for the change was that many respondents made mistakes in entering the dollar value of sales four quarters into the future. Some common mistakes included giving an annual rather than quarterly value for the firm's current or future sales level or failing to keep units consistent. In some cases, respondents reported current sales in units of dollars and future values in thousands or millions of dollars, at other times using different units across months. By asking for sales growth rates we created fewer opportunities for respondents to make such mistakes.

May 2019 onwards

In May 2019 we implemented a new round of changes to the SBU questionnaire. We eliminated the unit cost growth questions given our limited ability to track actual changes in unit costs and due to feedback from our respondents concerning that question. Several respondents have cited difficulty answering questions about unit costs. Service firms, in particular, cited 
confusion about this question, often saying that unit costs are more relevant for manufacturing. We therefore decided to concentrate on subjective probability distributions for future employment, sales, and capital expenditures, asking about only one of these topics in a given month. These changes expanded the number of respondents receiving questions about a given topic in a given month. Having a rotating panel of three questions rotated monthly also means that a given firm answers questions about a given topic once per quarter.

Starting in May 2019, we also include a question about the level of the stock of capital (i.e., property, plant and equipment) as part of the investment questionnaire. Responses to this question let us construct measures of current and future investment rates (I/K). 


\section{Figure B.1: October 2013 Trial Questions}

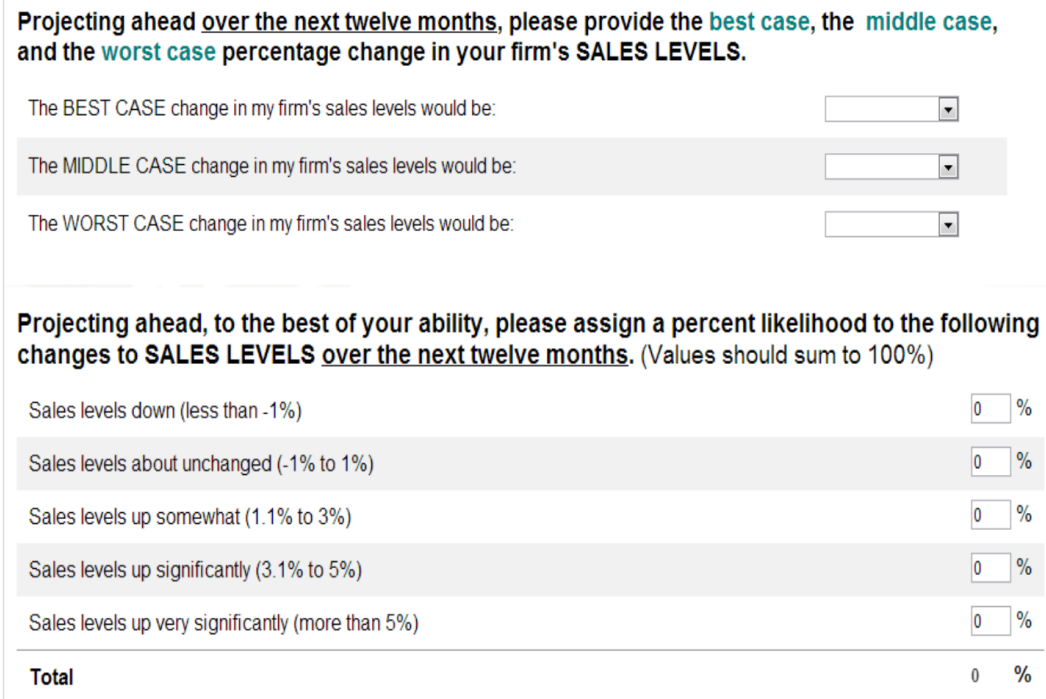

Notes: This figure shows screenshots from our first trial at eliciting subjective probabilities for future sales growth in October 2013. We performed an A/B test, giving half of the Atlanta Fed's BIE panel the threepoint question above and the other half the bottom question.

\section{Figure B.2: November 2013 Trial Questions}

\begin{abstract}
Projecting ahead over the next twelve months, please provide the best case, the middle case, and the worst case percentage change in your firm's SALES LEVELS.

(Note: "Best case" should correspond to about the top 10\% likelihood outcome and "worst case" the bottom 10\% likelihood outcome.)

The BEST CASE change in my firm's sales levels would be

The MIDDLE CASE change in my firm's sales levels would be

The WORST CASE change in my firm's sales levels would be
\end{abstract}

Projecting ahead over the next twelve months, please assign a percent likelihood to the following changes to SALES LEVELS. (Values should sum to $100 \%$ )

Sales levels down (less than $-5 \%$ )

Sales levels about unchanged $(-5 \%$ to $5 \%)$

Sales levels up somewhat (5.1\% to $15 \%)$

Sales levels up significantly ( $15.1 \%$ to $25 \%)$

Sales levels up very significantly (more than $25 \%$ )

Total
Notes: This figure shows screenshots from our November 2013 test, again attempting to elicit subjective probabilities for future sales growth. We again performed an A/B test, giving half of the Atlanta Fed's BIE panel the three-point question above and the other half the bottom question. 


\section{Figure B.3: December 2013 Trial Questions}

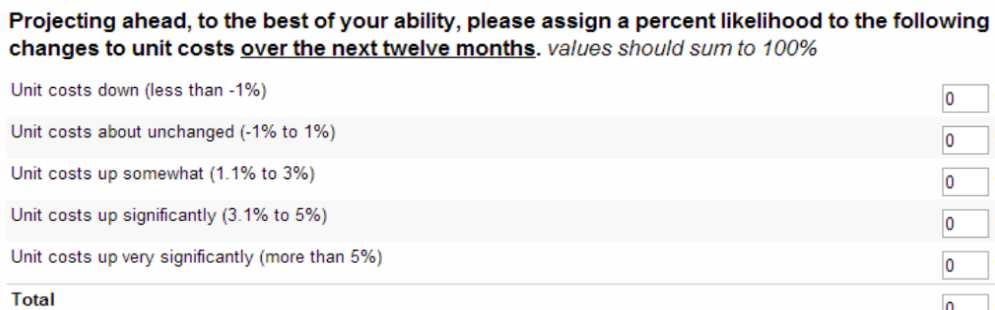

Projecting ahead, to the best of your ability, please provide your estimate of the worst case unit cost change, the most likely unit cost change, and the best case unit cost change your firm could experience over the next twelve months.

Note: "Best case" should correspond with the lowest $10 \%$ of possible unit cost changes, and "worst case" should correspond to the highest $10 \%$ of possible unit cost changes

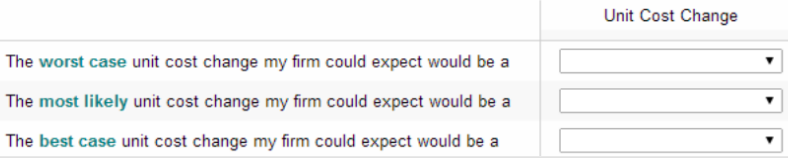

Notes: This figure shows screenshots from our December 2013 test, now attempting to elicit subjective probabilities for future unit cost growth. We again performed an A/B test, giving half of the Atlanta Fed's BIE panel the five-bin question above and the other half the bottom question with three support points.

\section{Figure B.4: January 2014 Trial Question}

Projecting ahead over the next twelve months, please provide the approximate low case, medium case, and high case percentage change in your firm's SALES LEVELS.

The LOW CASE change in my firm's sales levels would be:
The MEDIUM CASE change in my firm's sales levels would be:
The HIGH CASE change in my firm's sales levels would be:

Please assign a percentage likelihood to the low case, medium case, and high case percentage SALES LEVEL changes you selected above.

— LOW CASE: The likelihood of realizing about a $0 \%$ change in sales levels

— MEDIUM CASE: The likelihood of realizing about a $2 \%$ change in sales levels

- HIGH CASE: The likelihood of realizing about a $4 \%$ change in sales levels
Notes: This figure shows screenshots from our January 2014 test question, again eliciting subjective probabilities for future sales growth. We sent the same question to all of the Atlanta Fed's BIE panel of respondents. This new question has two parts: the top asks firms to provide numerical outcomes for their "low", "medium", and "high" outcomes and the bottom asks for probabilities. 


\section{Figure B.5: February 2014 Trial Question}

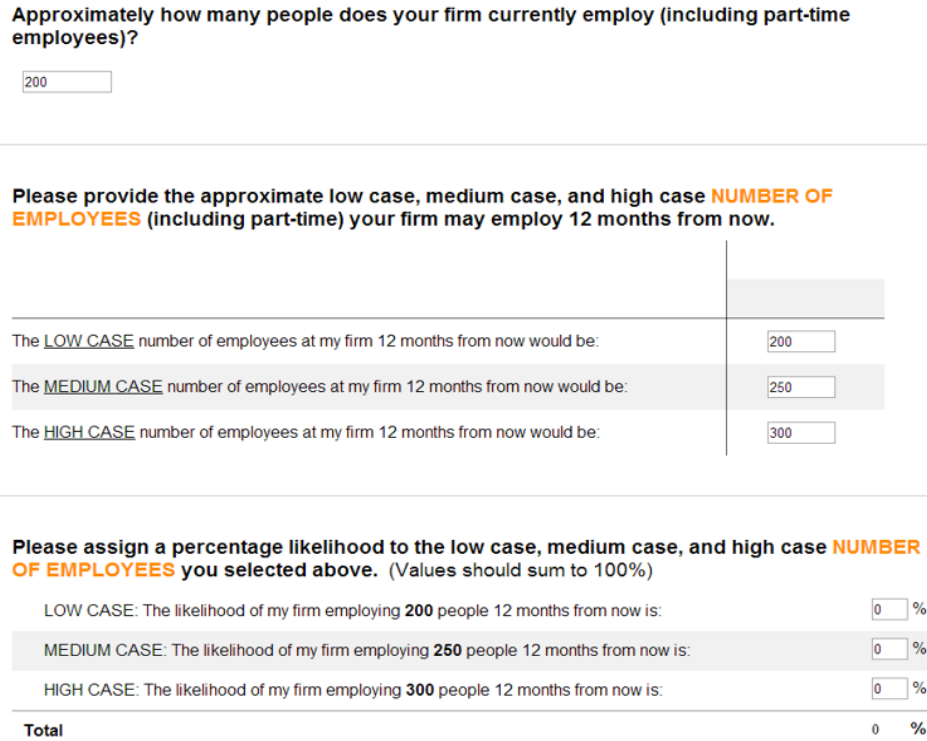

Approximately how many people does your firm currently employ (including part-time employees)?

200

Please provide the approximate low case, medium case, and high case NUMBER OF EMPLOYEES (including part-time) your firm may employ 12 months from now.

Please assign a percentage likelihood to the low case, medium case, and high case NUMBER OF EMPLOYEES you selected above. (Values should sum to $100 \%$ )

LOW CASE: The likelihood of my firm employing 200 people 12 months from now is:

MEDIUM CASE: The likelihood of my firm employing 250 people 12 months from now is:

HIGH CASE: The likelihood of my firm employing 300 people 12 months from now is:

Total

Notes: This figure shows screenshots from our February 2014 test question, now eliciting subjective probability distributions for future employment. We sent the same question to all of the Atlanta Fed's BIE panel of respondents. This new question has three parts: first it asks for current employment levels, then asks for numerical outcomes for the "low", "medium", and "high" outcomes, and finally the bottom asks for probabilities for those outcomes.

\section{Figure B.6: March 2014 Trial Question}

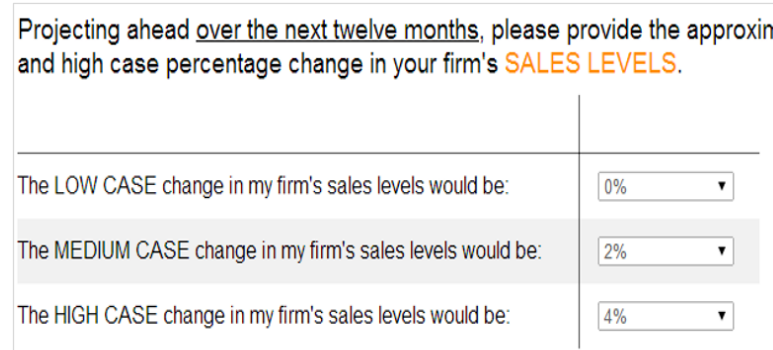

Please assign a percentage likelihood to the low case, medium case, and high case percentage SALES LEVEL changes you selected above.

— LOW CASE: The likelihood of realizing about a $0 \%$ change in sales levels

— MEDIUM CASE: The likelihood of realizing about a $2 \%$ change in sales levels

- $\%$ HIGH CASE: The likelihood of realizing about a $4 \%$ change in sales levels
Notes: This figure shows screenshots from our March 2014 test question, which repeated the January 2014 experiment and elicited subjective probabilities for future sales growth. We sent the same question to all of the Atlanta Fed's BIE panel of respondents. 


\section{Figure B.7: April 2014 Trial Question}

\begin{tabular}{l} 
Projecting ahead over the next twelve months, please provide an approximate percentage \\
change in your firm's SALES LEVELS for the following scenarios: \\
The WORST CASE change in my firm's sales levels would be: \\
The LOW CASE change in my firm's sales levels would be: \\
The MEDIUM CASE change in my firm's sales levels would be: \\
The HIGH CASE change in my firm's sales levels would be: \\
The BEST CASE change in my firm's sales levels would be: \\
Please assign a percentage likelihood to the percentage SALES LEVEL changes you selected \\
above. (Values should sum to $100 \%$ ) \\
WORST CASE: The likelihood of realizing about a less than -24\% change in sales levels would be \\
LOW CASE: The likelihood of realizing about a $-17 \%$ change in sales levels would be \\
MEDIUM CASE: The likelihood of realizing about a $18 \%$ change in sales levels would be \\
HIGH CASE: The likelihood of realizing about a $19 \%$ change in sales levels would be \\
BEST CASE: The likelihood of realizing about a $33 \%$ change in sales levels would be \\
\hline Total
\end{tabular}

Notes: This figure shows screenshots from our April 2014 test question, which extended our January and March 2014 experiments to a five-point format, again eliciting subjective probabilities for future sales growth. We sent the same question to all of the Atlanta Fed's BIE panel of respondents.

\section{Figure B.8: May 2014 Trial Question}

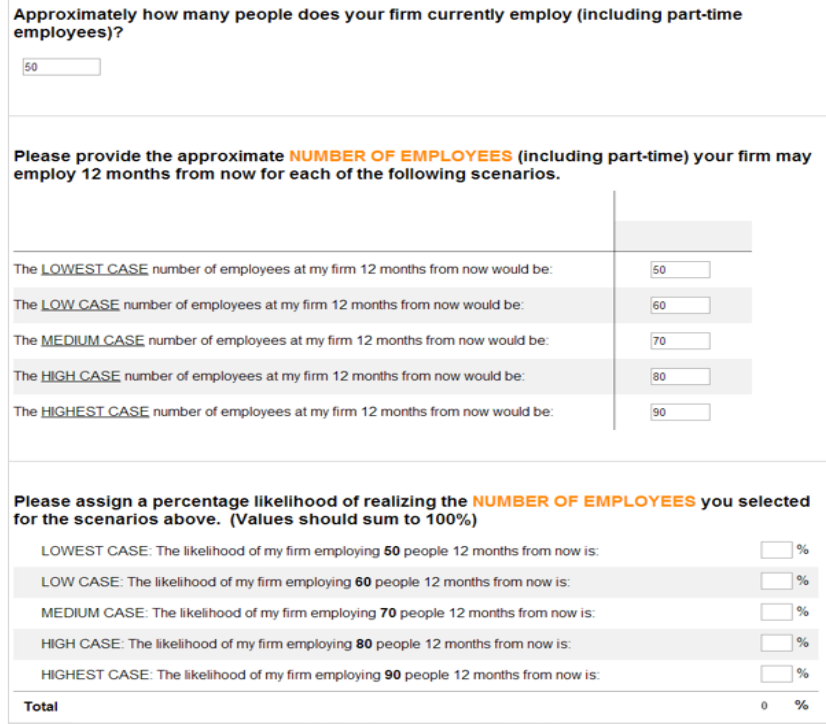

Notes: This figure shows screenshots from our May 2014 test question, which extended our February 2014 test for eliciting subjective probabilities for future employment levels, now using a five-point format. We sent the same question to all of the Atlanta Fed's BIE panel of respondents. 


\section{Figure B.9: June 2014 Trial Question}

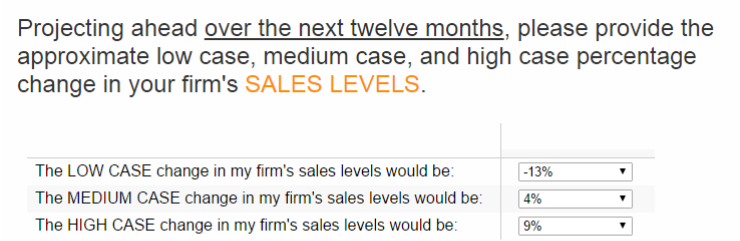

Please assign a percentage likelihood to the low case, medium case, and high case percentage SALES LEVEL changes you selected above. (Values should sum to $100 \%$ )

LOW CASE: The likelihood of realizing about a $-13 \%$ change in sales levels MEDIUM CASE: The likelihood of realizing about a $4 \%$ change in sales levels

HIGH CASE: The likelihood of realizing about a $9 \%$ change in sales levels

Projecting ahead over the next twelve months, please provide the approximate best case and worst case percentage change in your firm's SALES LEVELS.

\begin{abstract}
Notes: This figure shows screenshots from our June 2014 test question, which replicated the question from January and March 2014, using a three-point design to elicit subjective probabilities for future sales growth levels. Then we additionally asked respondents for estimates of their "worst" and "best" case scenarios. We sent the same question to all of the Atlanta Fed's BIE panel of respondents.
\end{abstract}

\section{Figure B.10: July 2014 Questions (Asking for five vs. three support points)}

Notes: This figure shows screenshots from our July 2014 test questions, in which we A/B tested three- and five-support point designs to elicit subjective probability distributions about employment, prices and sales. July 2014 was the first month in which we tested our questions on a newly-recruited panel of firms for the SBU specifically. We randomly split the panel into two sub-groups, with the first group assigned the three-point question and the second assigned the five-point question. 


\section{B.10a. Employment Questions}

Please provide the approximate NUMBER OF EMPLOYEES (including parttime) your firm may employ 12 months from now for each of the following scenarios.
The LOWEST CASE number of employees at my firm 12 months from now would be The LOW CASE number of employees at my firm 12 months from now would be The MEDIUM CASE number of employees at my firm 12 months from now would be. The HIGH CASE number of employees at my firm 12 months from now would be. The HIGHEST CASE number of employees at my firm 12 months from now would be

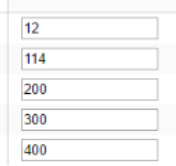

Please assign a percentage likelihood of realizing the NUMBER OF EMPLOYEES you selected above. (Values should sum to $100 \%$ )
Projecting ahead over the next twelve months, please provide the approximate low case, medium case, and high case NUMBER OF EMPLOYEES (including part-time) you may have.

The LOW CASE number of employees my firm may have 12 months from now would be: The MEDIUM CASE number of employees my firm may have 12 months from now would

The HIGH CASE number of employees my fim may have 12 months from now would be:
400

Please assign a percentage likelihood to the low case, medium case, and high case NUMBER OF EMPLOYEES you selected above. (Responses should sum to 100)

LOW CASE: The likelihood of my fim employing 250 employees 12 months from now is:

MEDIUM CASE: The likelihood of my firm employing 300 employees 12 months from now is:

HIGH CASE: The likelihood of my firm employing 400 employees 12 months from now is:

$0 \%$

\section{B.10b. Prices}

Projecting ahead over the next twelve months, for each of the following scenarios please provide an approximate percentage change in the AVERAGE PRICE you charge:

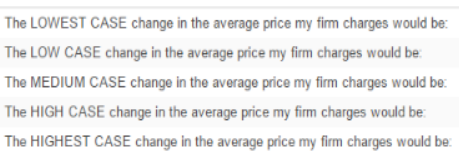

Please assign a percentage likelihood to the percentage AVERAGE PRICE changes you selected above. (Values should sum to $100 \%$ )

LOWEST CASE: The likelihood of realizing about a $\%$ change in average price would be

LOW CASE: The likelihood of realizing about a $1 \%$ change in average price would be

MEDIUM CASE: The likelihood of realizing about a $2 \%$ change in average price would be

HIGH CASE: The likelihood of realizing about a $3 \%$ change in average price would be

HIGHEST CASE: The likelihood of realizing about a $4 \%$ change in average price would be

Total

Projecting ahead over the next twelve months, please provide the approximate low case, medium case, and high case percentage change in the AVERAGE PRICE you charge.

The LOW CASE change in the average price my firm charges would be:
The MEDIUM CASE change in the average price my firm charges would
be:
The HIGH CASE change in the average price my firm charges would be:

Please assign a percentage likelihood to the low case, medium case, and high case percentage AVERAGE PRICE changes you selected above. (Values should sum to $100 \%)$

LOW CASE: The likellihood of my firm realizing about a $0 \%$ change in its average price would be MEDIUM CASE: The likelihood of my firm realizing about a $5 \%$ change in its average price would be HIGH CASE: The likelihood of my firm realizing about a $7 \%$ change in its average price would be

Total 


\section{B.10c. Sales Growth}

Projecting ahead over the next twelve months, for each of the following scenarios please provide an approximate percentage change in your firm's SALES REVENUE:
The LOWEST CASE change in my firm's sales levels would be:

The LOW CASE change in my firm's sales levels would be:

The MEDIUM CASE change in my firm's sales levels would be

The HIGH CASE change in my firm's sales levels would be

The HIGHEST CASE change in my firm's sales levels would be

$$
\begin{aligned}
& \begin{array}{|l|}
\hline 0 \% \quad \text { - } \\
\hline 1 \% \quad \\
\hline
\end{array} \\
& 2 \% \text { - } \\
& \begin{array}{|l|}
3 \% \quad \text { - } \\
\hline 4 \% \quad \text { - }
\end{array}
\end{aligned}
$$

Projecting ahead over the next twelve months, please provide the approximate low case, medium case, and high case percentage change in your firm's SALES REVENUE.

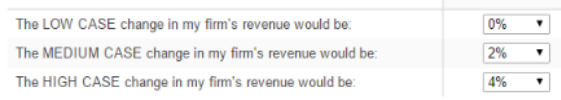

Please assign a percentage likelihood to the low case, medium case, and high case percentage changes in your firm's SALES REVENUE you selected above. (Values should sum to $100 \%$ )

LOW CASE: The likellihood of my firm reallizing about a $0 \%$ change in revenue

MEDIUM CASE: The likelihood of my firm realizing about a $2 \%$ change in revenue

HIGH CASE: The likelihood of my firm realizing about a $4 \%$ change in revenue

Please assign a percentage likelihood to the percentage SALES REVENUE changes you selected above. (Values should sum to 100\%)

LOWEST CASE: The likelihood of realizing about a $0 \%$ change in sales levels would be

LOW CASE: The likelihood of realizing about a $1 \%$ change in sales levels would be

MEDIUM CASE: The likelihood of realizing about a $2 \%$ change in sales levels would be

HIGH CASE: The likelihood of realizing about a $3 \%$ change in sales levels would be

HIGHEST CASE: The likelihood of realizing about a $4 \%$ change in sales levels would be

Total

\section{Figure B.11: Additional SBU Questions During $1^{\text {st }}$ Generation (August 2014-August 2016) B.11a. Profit Margins}

Currently, what is your AVERAGE PROFIT MARGIN, considering all of your products and services (in percentage terms)?

Looking back, 12 months ago, what was your AVERAGE PROFIT MARGIN (in percentage terms)? 
Looking ahead, 12 months from now, what AVERAGE PROFIT MARGIN would you assign to each of the following scenarios (in percentage terms)?

\begin{tabular}{l|l|}
\hline & \\
\hline Our LOWEST-CASE average profit margin would be: & \\
Our LOW-CASE average profit margin would be: & \\
Our MEDIUM-CASE average profit margin would be: & \\
Our HIGH-CASE average profit margin would be: & \\
Our HIGHEST-CASE average profit margin would be:
\end{tabular}

Please assign a percentage likelihood to the AVERAGE PROFIT
MARGINS you entered above. (Values should sum to 100\%)
LOWEST CASE: The likelihood of about a 1 percent average profit margin would be:
LOW CASE: The likelihood of about a 2 percent average profit margin would be:
MEDIUM CASE: The likelihood of about a 3 percent average profit margin would be:
HIGH CASE: The likelihood of about a 4 percent average profit margin would be:
HIGHEST CASE: The likelihood of about a 5 percent average profit margin would be:
Total

\section{B.11b. Prices}

Looking back, from 12 months ago to now, what was the approximate percentage change in the AVERAGE PRICE you charge, considering all of your products and services?

Looking ahead, from now to 12 months from now, what approximate percentage change in AVERAGE PRICE would you assign to each of the following scenarios? 

The LOW-CASE percentage change in the average price you charge would be: The MEDIUM-CASE percentage change in the average price you charge would be: The HIGH-CASE percentage change in the average price you charge would be: The HIGHEST-CASE percentage change in the average price you charge would be:

\begin{tabular}{|l|}
\hline 1 \\
\hline 2 \\
\hline 3 \\
\hline 4 \\
\hline 5 \\
\hline
\end{tabular}

Please assign a percentage likelihood to the AVERAGE PRICE changes you entered above. (Values should sum to $100 \%$ )

LOWEST CASE: The likelihood of about a 1 percent change in the average price you charge would be: LOW CASE: The likelihood of about a 2 percent change in the average price you charge would be: MEDIUM CASE: The likelihood of about a $\mathbf{3}$ percent change in the average price you charge would be: HIGH CASE: The likelihood of about a 4 percent change in the average price you charge would be: HIGHEST CASE: The likelihood of about a $\mathbf{5}$ percent change in the average price you charge would be: Total

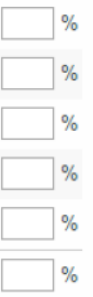




\section{Table B.1: Summary of Tests for Developing the Survey of Business Uncertainty}

\begin{tabular}{|c|c|c|c|c|}
\hline Panel & Date & Variable(s) & Abbreviated description & Description \\
\hline \multirow{9}{*}{ 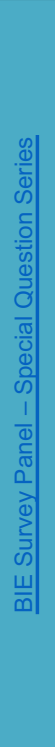 } & Oct-13 & sales levels & $\begin{array}{l}\text { A/B test. three-estimate } \\
\text { and five-binned range } \\
\text { versions. }\end{array}$ & $\begin{array}{l}\text { Participants were randomly assigned to one of two groups. Group } 1 \text { received a question eliciting the "best," "most } \\
\text { likely," and "worst" case change in sales levels over the next } 12 \text { months. A drop-down box was provided with } \\
\text { estimates ranging from }-15 \% \text { to } 30 \% \text {. Group } 2 \text { received a question asking respondents to assign a likelihood to five } \\
\text { potential percentage sales level change ranges (from "less than }-1 \% \text { " to "more than } 5 \% \text { ") over the next } 12 \text { months. }\end{array}$ \\
\hline & Nov-13 & sales levels & A/B test. & $\begin{array}{l}\text { Participants were randomly assigned to one of two groups. Group } 1 \text { received a question eliciting the "best," "most } \\
\text { likely," and "worst" case change in sales levels over the next twelve months. For each estimate a drop-down box was } \\
\text { provided with options ranging from -15\% to } 30 \% \text {. A note indicating "best" and "worst" case scenarios should be } \\
\text { associated with a } 10 \% \text { chance of occurrence was includeC. Group } 2 \text { received a question asking respondents to assign } \\
\text { a likelihood to five potential percentage sales level change ranges (ranging from "less than -5\%" to "more than } 25 \% " \text { ) } \\
\text { over the next } 12 \text { months. }\end{array}$ \\
\hline & Dec-13 & unit costs & A/B test & $\begin{array}{l}\text { Participants were randomly assigned to one of two groups. Group } 1 \text { received a question eliciting the "best," "middle," } \\
\text { and "worst" case percentage change in unit costs over the next } 12 \text { months. Group } 2 \text { received a question asking } \\
\text { respondents to assign a likelihood to five potential percentage unit cost change ranges (from "less than }-1 \% \text { " to "more } \\
\text { than } 5 \% " \text { ) over the next } 12 \text { months. }\end{array}$ \\
\hline & Jan-14 & sales levels & three estimates & $\begin{array}{l}\text { Participants received a two-part question. Part one elicited the expected "low," "middle," and "high" case changes in } \\
\text { sales levels over the next twelve months. Part two asked respondents to assign a likelihood of occurrence for each of } \\
\text { the three scenarios. }\end{array}$ \\
\hline & Feb-14 & $\begin{array}{l}\text { number of } \\
\text { employees }\end{array}$ & three estimates & $\begin{array}{l}\text { Participants received a two-part question. Part one elicited the expected "low," "middle," and "high" case number of } \\
\text { employees twelve months ahead. Part two asked respondents to assign a likelihood of occurrence for each of the } \\
\text { three scenarios. }\end{array}$ \\
\hline & Mar-14 & sales levels & three estimates & Repeat of the January 2014 question. \\
\hline & Apr-14 & sales levels & five estimates & $\begin{array}{l}\text { The same question as in January and March } 2014 \text { with the addition of a "worst case" and "best case" scenario for a } \\
\text { total of five response categories. }\end{array}$ \\
\hline & May-14 & $\begin{array}{l}\text { number of } \\
\text { employees }\end{array}$ & five estimates & $\begin{array}{l}\text { The same question as in February } 2014 \text { with the addition of a "worst case" and "best case" scenario for a total of five } \\
\text { response categories. }\end{array}$ \\
\hline & Jun-14 & sales levels & $\begin{array}{l}\text { three estimates with a } \\
\text { best case/worst case } \\
\text { follow-up }\end{array}$ & $\begin{array}{l}\text { Repeat of the January } 2014 \text { question with a follow-up question asking for the "best case" and "worst case" scenarios } \\
\text { without a likelihood assignment. }\end{array}$ \\
\hline
\end{tabular}

\begin{tabular}{|c|c|c|c|c|c|}
\hline Panel & Date & No. of Groups & Variable(s) & Notes & Description \\
\hline \multirow{3}{*}{$\begin{array}{c}\bar{\Phi} \\
\stackrel{\bar{\sigma}}{\circ} \\
0\end{array}$} & Jul-14 & 2 & $\begin{array}{c}\text { number of employees, average } \\
\text { price, sales revenue }\end{array}$ & $\begin{array}{l}\text { A/B Test }-5 \text { estimate and } 3 \\
\text { estimate versions with drop down } \\
\text { boxes for estimates and open text } \\
\text { boxes for likelihoods }\end{array}$ & $\begin{array}{l}\text { Participants were randomly assigned to one of two groups. In each group, } \\
\text { espondents received a two-part question for each variable. Grop 1: Part one } \\
\text { elicited the "high," "medium," and "low" case change in each variable over } \\
\text { the next } 12 \text { months. Part two asked respondents to assign a likelihood to } \\
\text { each of these scenarios. Group 2: Same format as Group } 1 \text { with two } \\
\text { additional scenarios eliciting the "lowest case" and "highest case." }\end{array}$ \\
\hline & Aug-14 & 2 & $\begin{array}{l}\text { sales revenue, average price, } \\
\text { number of employees, unit } \\
\text { cost, capital investment, profit } \\
\text { margin }\end{array}$ & $\begin{array}{l}\text { five estimates with drop down box } \\
\text { for estimates and open text box } \\
\text { for likelihoods }\end{array}$ & $\begin{array}{l}\text { Participants received a two-part question for each variable. Part one elicited } \\
\text { the "highest," "high," "medium," "low," and "lowest" case change in each } \\
\text { variable over the next } 12 \text { months. Part two asked respondents to assign a } \\
\text { likelihood to each of these scenarios. }\end{array}$ \\
\hline & Sep-14 & 2 & $\begin{array}{c}\text { sales revenue, average prices, } \\
\text { unit cost, capital investment }\end{array}$ & $\begin{array}{l}\text { five estimates with open text } \\
\text { boxes for estimates and } \\
\text { likelihoods }\end{array}$ & $\begin{array}{l}\text { Participants received a two-part question for each variable. Part one elicited } \\
\text { the "highest," "high," "medium," "low," and "lowest" case change in each } \\
\text { variable over the next } 12 \text { months. Part two asked respondents to assign a } \\
\text { likelihood to each of these scenarios. }\end{array}$ \\
\hline \multirow{3}{*}{ 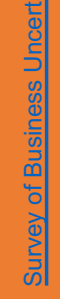 } & $\begin{array}{c}\text { Oct-14 } \\
\text { to } \\
\text { Jan-15 }\end{array}$ & 3 & $\begin{array}{c}\text { sales revenue, average price, } \\
\text { number of employees, unit } \\
\text { cost, capital investment, profit } \\
\text { margin }\end{array}$ & $\begin{array}{l}\text { five estimates with open text } \\
\text { boxes for estimates and } \\
\text { likelihoods }\end{array}$ & $\begin{array}{l}\text { Participants received a two-part question for each variable. Part one elicited } \\
\text { the "highest," "high," "medium," "low," and "lowest" case change in each } \\
\text { variable over the next } 12 \text { months. Part two asked respondents to assign a } \\
\text { likelihood to each of these scenarios. }\end{array}$ \\
\hline & $\begin{array}{c}\text { Feb-15 } \\
\text { to } \\
\text { Oct-15 }\end{array}$ & 3 & $\begin{array}{c}\text { sales revenue, average price, } \\
\text { number of employees, unit } \\
\text { cost, capital investment, profit } \\
\text { margin } \\
\end{array}$ & $\begin{array}{l}\text { five estimates with open text } \\
\text { boxes for estimates and } \\
\text { likelihoods }\end{array}$ & $\begin{array}{l}\text { Participants received a two-part question for each variable. Part one elicited } \\
\text { the "highest," "high," "medium," "low," and "lowest" case change in each } \\
\text { variable over the next } 12 \text { months. Part two asked respondents to assign a } \\
\text { likelihood to each of these scenarios. }\end{array}$ \\
\hline & $\begin{array}{c}\text { Nov-15 } \\
\text { to Jan- } \\
16\end{array}$ & 6 & $\begin{array}{c}\text { sales revenue, average price, } \\
\text { number of employees, unit } \\
\text { cost, capital investment, profit } \\
\text { margin }\end{array}$ & $\begin{array}{l}\text { five estimates with open text } \\
\text { boxes for estimates and } \\
\text { likelihoods }\end{array}$ & $\begin{array}{l}\text { Participants received a two-part question for each variable. Part one elicited } \\
\text { the "highest," "high," "middle," "low," and "lowest" case change in each } \\
\text { variable over the next } 12 \text { months. Part two asked respondents to assign a } \\
\text { likelihood to each of these scenarios. }\end{array}$ \\
\hline & $\begin{array}{c}\text { Feb-16 } \\
\text { to Aug- } \\
16\end{array}$ & 6 & $\begin{array}{c}\text { sales revenue, average price, } \\
\text { number of employees, unit } \\
\text { cost, capital investment, profit } \\
\text { margin }\end{array}$ & $\begin{array}{l}\text { five estimates with open text } \\
\text { boxes for estimates and } \\
\text { likelihoods }\end{array}$ & $\begin{array}{l}\text { Participants received a two-part question for each variable. Part one elicited } \\
\text { the "highest," "high," "middle," "low," and "lowest" value for each variable } \\
\text { over the next } 12 \text { months. Part two asked respondents to assign a likelihood } \\
\text { to each of these scenarios. }\end{array}$ \\
\hline & $\begin{array}{c}\text { Sep-16 } \\
\text { to } \\
\text { Present }\end{array}$ & 2 & $\begin{array}{l}\text { sales revenue, average unit } \\
\text { cost, capital expenditures, } \\
\text { number of employees }\end{array}$ & $\begin{array}{l}\text { five estimates with open text } \\
\text { boxes for estimates and } \\
\text { likelihoods }\end{array}$ & $\begin{array}{l}\text { Participants received a two-part question for each variable. Part one elicited } \\
\text { the "highest," "high," "middle," "low," and "lowest" value for each variable } \\
\text { over the next } 12 \text { months. Part two asked respondents to assign a likelihood } \\
\text { to each of these scenarios. }\end{array}$ \\
\hline
\end{tabular}




\section{Additional Empirical Results: Microdata}

\section{C.1 Core results focusing on employment growth expectations and uncertainty}

- The next few slides replicate some of the core results about the SBU microdata focusing on employment growth rather than sales growth expectations and uncertainty.

- Broadly speaking the results are the same whether we focus on sales or employment. In several cases they are sharper for employment, which we believe owes to less measurement error in employment expectations and realizations. 


\section{Figure C.1: Subjective Expectations Predict Realizations}

Figure C.1a

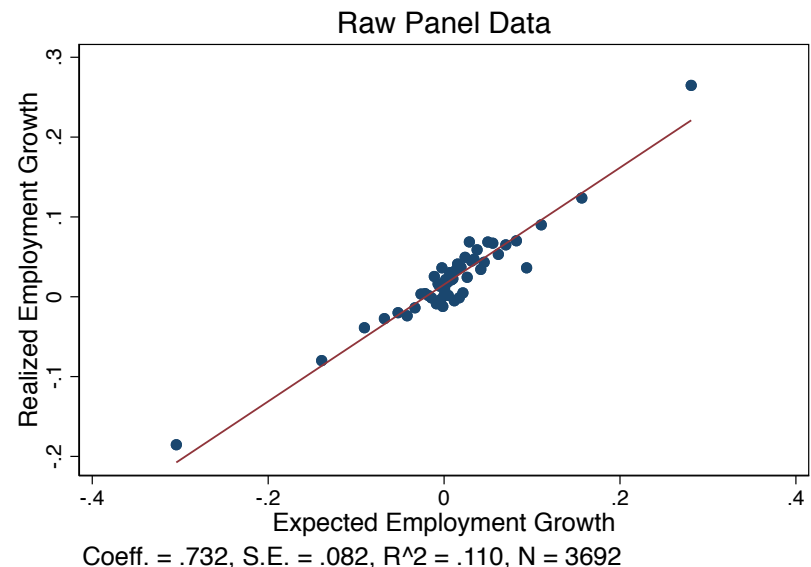

Figure C.1c

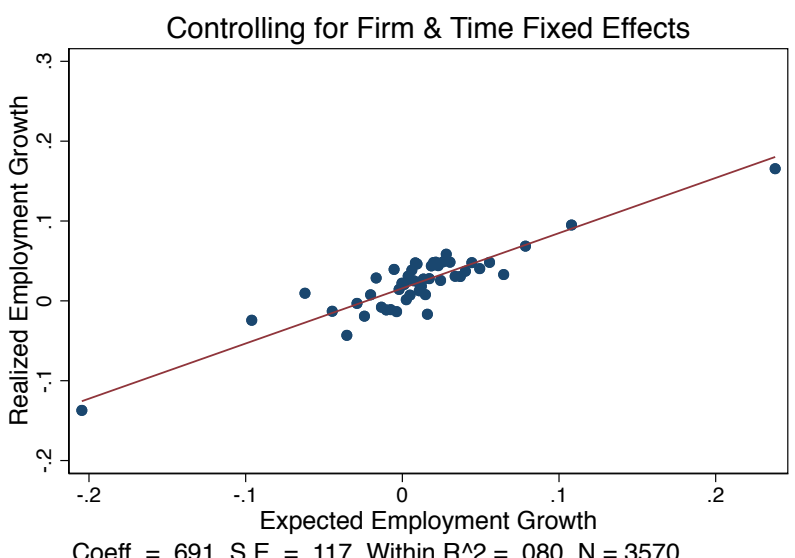

Figure C.1b

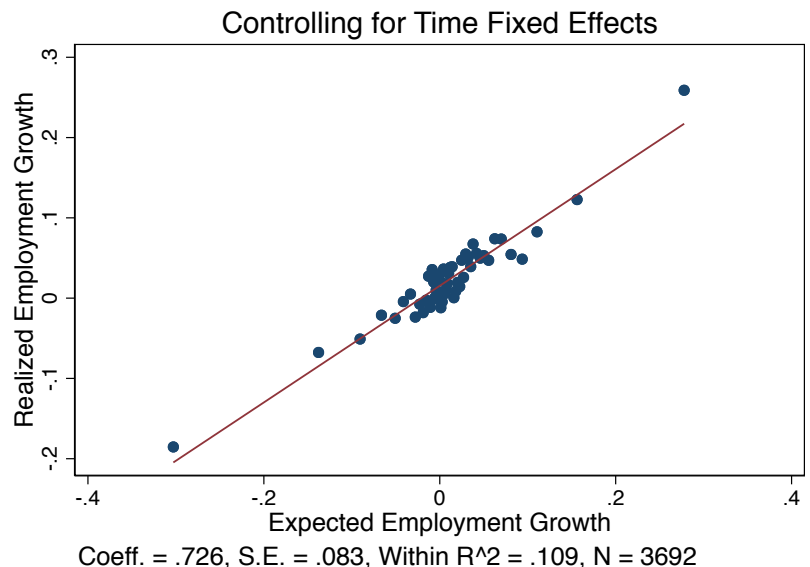

Coeff. $=.726$, S.E. $=.083$, Within $\mathrm{R}^{\wedge} 2=.109, \mathrm{~N}=3692$
Figure C.1d

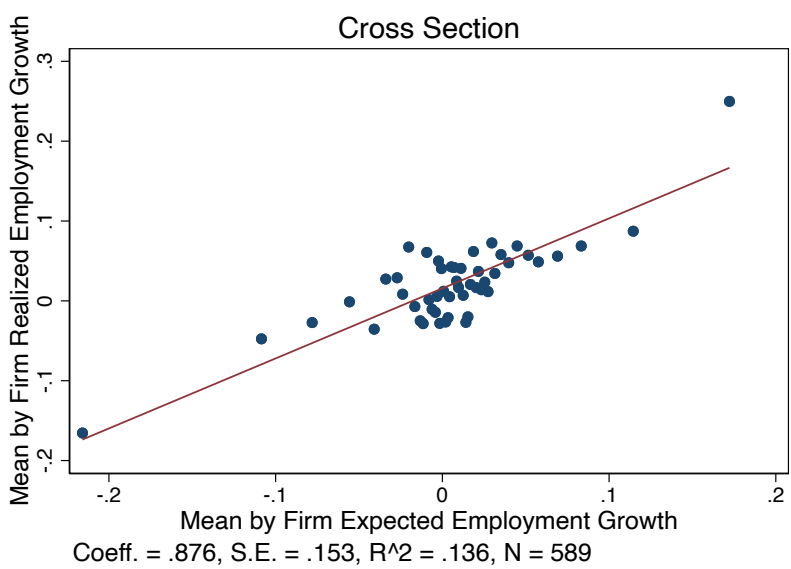

Notes: This figure shows bin-scatter plots of employment growth expectations for the next 12 months on the horizontal axis against measured employment growth over the ensuing 12 months on the vertical axis. Figure B.1a shows the relationship in the raw panel data. Figure B.1b controls for time effects. Figure B.1c controls for both firm and time fixed effects. Figure B.1d shows the relationship in the cross section, plotting the mean-by-firm expected employment growth on the horizontal axis and mean-by-firm realized employment growth on the vertical axis. The reported statistics below each figure correspond to the OLS regression in the underlying microdata, reporting firm-clustered standard errors. Data are from all waves of the SBU from 10/2014 to 10/2019. 


\section{Figure C.2: Subjective Uncertainty Predicts Absolute Forecast Errors}

Figure C.2a

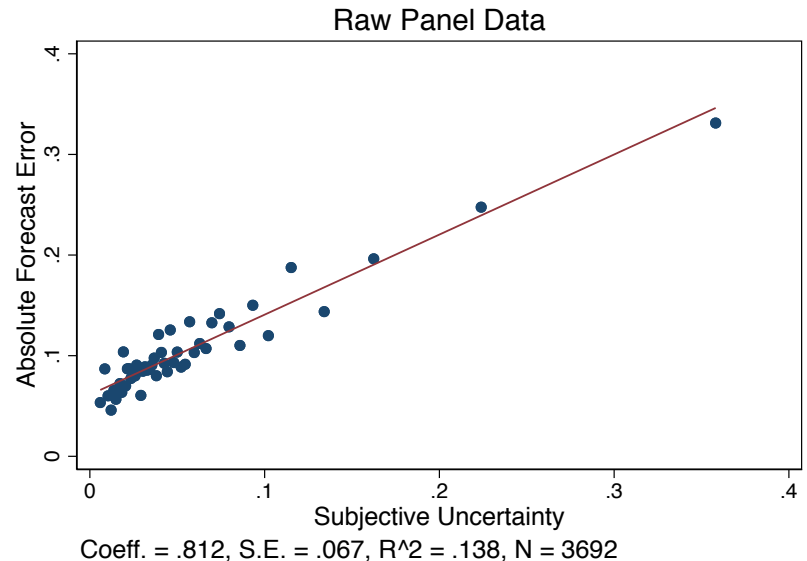

Coeff. $=.812$, S.E. $=.067, \mathrm{R}^{\wedge} 2=.138, \mathrm{~N}=3692$
Figure C.2b

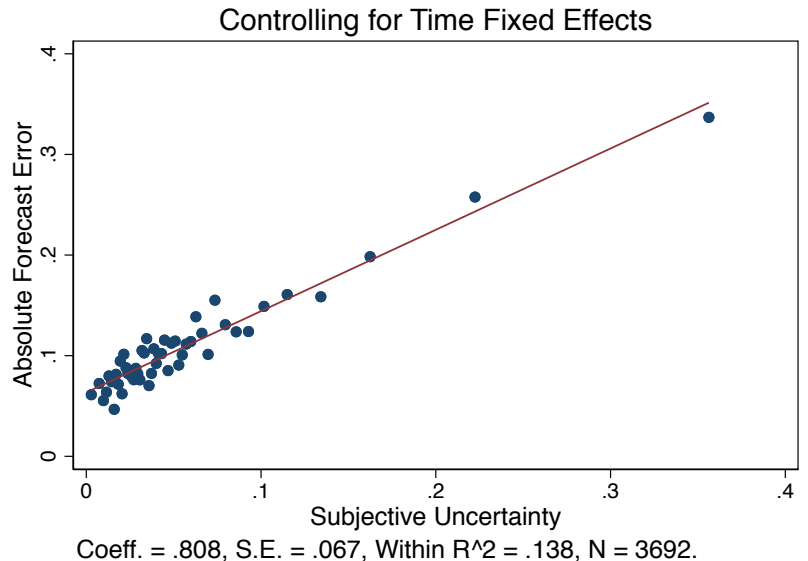

Coeff. $=.808$, S.E. $=.067$, Within $\mathrm{R}^{\wedge} 2=.138, \mathrm{~N}=3692$.
Figure C.2c

Controlling for Firm \& Time Fixed Effects

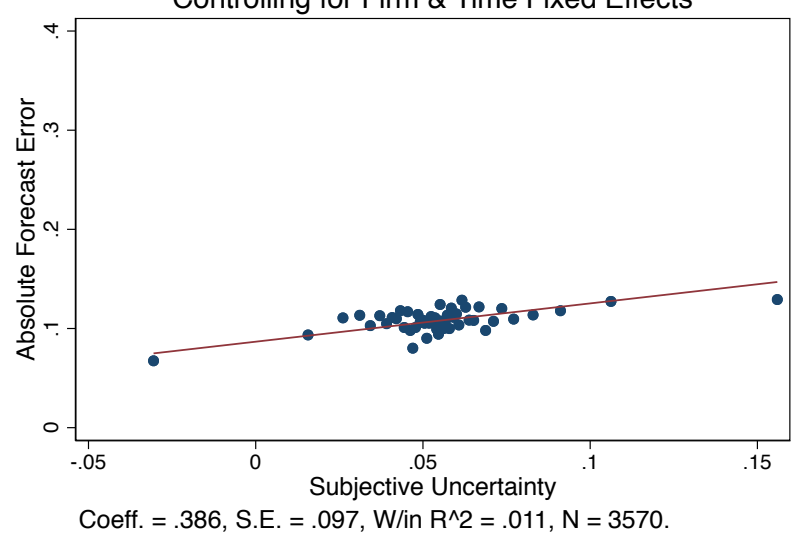

Figure C.2d

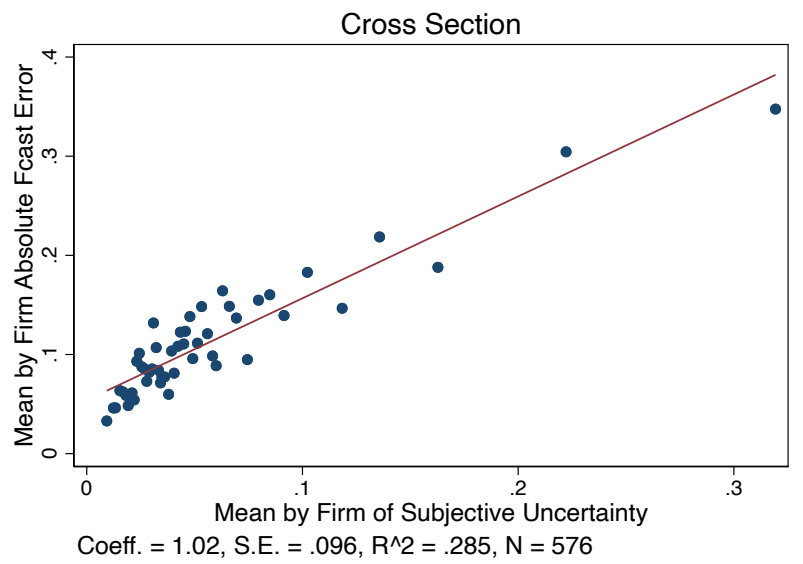

Notes: This figure shows bin-scatter plots of subjective uncertainty about the firm's employment growth for the next 12 months on the horizontal axis, against the respondent's absolute forecast error for employment growth over the ensuing 12 months on the vertical axis. Figure B.2a shows the relationship in the raw panel data. Figure B.2b controls for time effects. Figure B.2c also controls for firm effects. Figure B2.d shows the relationship in the cross section, plotting mean-by-firm subjective uncertainty on the horizontal axis against mean-by-firm absolute forecast errors on the vertical axis. The statistics below each figure correspond to the population OLS regression. Data are from all waves of the SBU from 10/2014 to 10/2019. 


\section{Figure C.3: Uncertainty and subsequent expectations revisions}

Figure C.3a

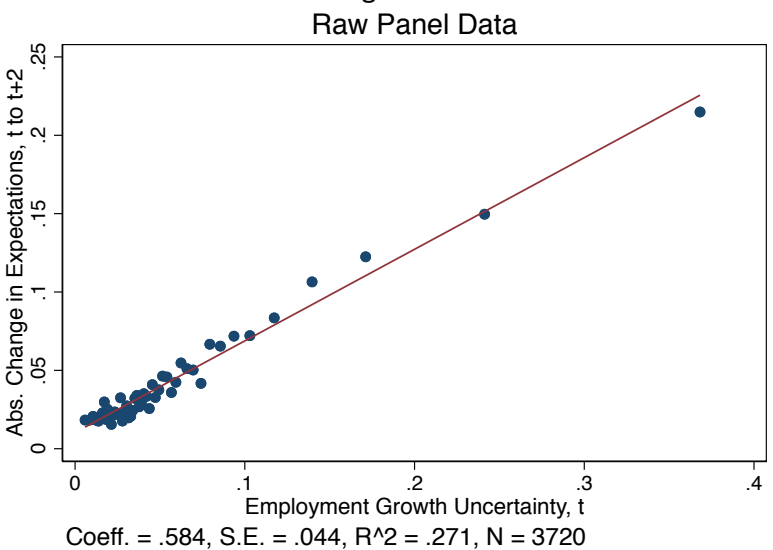

Figure C.3b

Controlling for Firm \& Time Fixed Effects

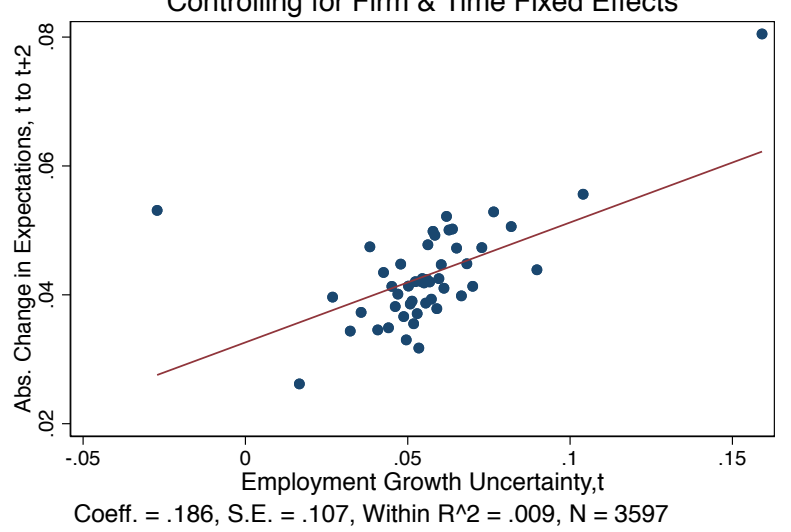

Notes: This figure shows two bin-scatter plots. On the horizontal axis, both show 50 quantiles of subjective uncertainty for employment growth over the next 12 months, measured in month $t$. Both have on the vertical axis the absolute value of the change in employment growth expectations (looking for 12 months ahead) from months $t$ to $t+2$ (or $t+3$ ). On the left, we show the relationship in the raw panel data, while on the right we show the relationship controlling for firm and time fixed effects. We report the underlying firm-level regressions with firm-clustered standard errors at the bottom of each figure, using SBU data from 10/2014 to 10/2019.

\section{Figure C.4: Uncertainty and subsequent uncertainty revisions}

Figure C.4a

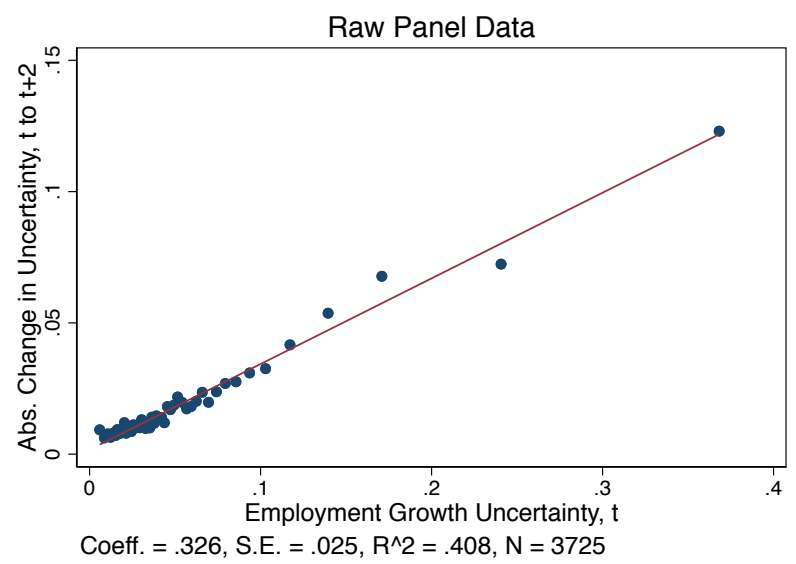

Figure C.4b

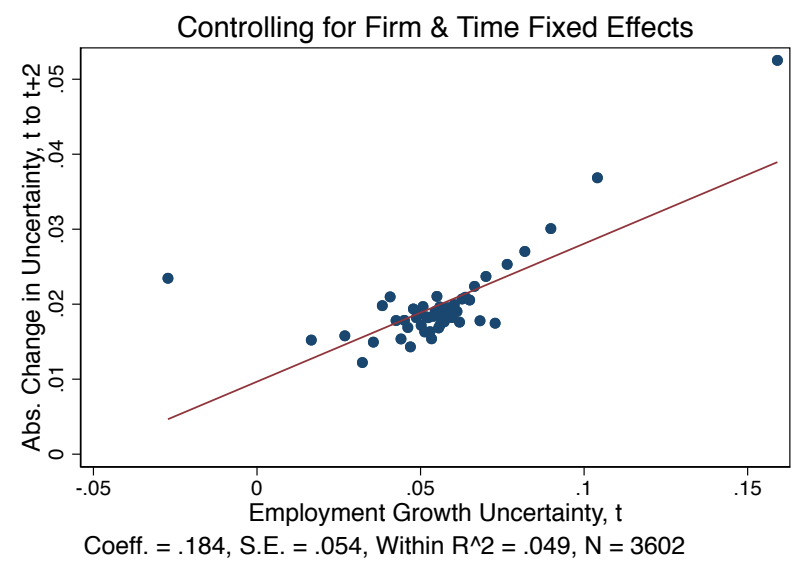

Notes: This figure shows two bin-scatter plots. On the horizontal axis, both show 50 quantiles of subjective uncertainty for employment growth over the next 12 months, measured in month $t$. Both have on the vertical axis the absolute value of the change in employment growth uncertainty from months $t$ to $t+2$ (or $t+3$ ). On the left, we show the relationship in the raw panel data, while on the right we show the relationship controlling for firm and time fixed effects. We report the underlying firm-level regressions with firm-clustered standard errors at the bottom of each figure, using SBU data from 10/2014 to 10/2019. 


\section{Figure C.5: Past sales growth and recent forecast revisions predict higher subjective uncertainty}

Figure C.5a

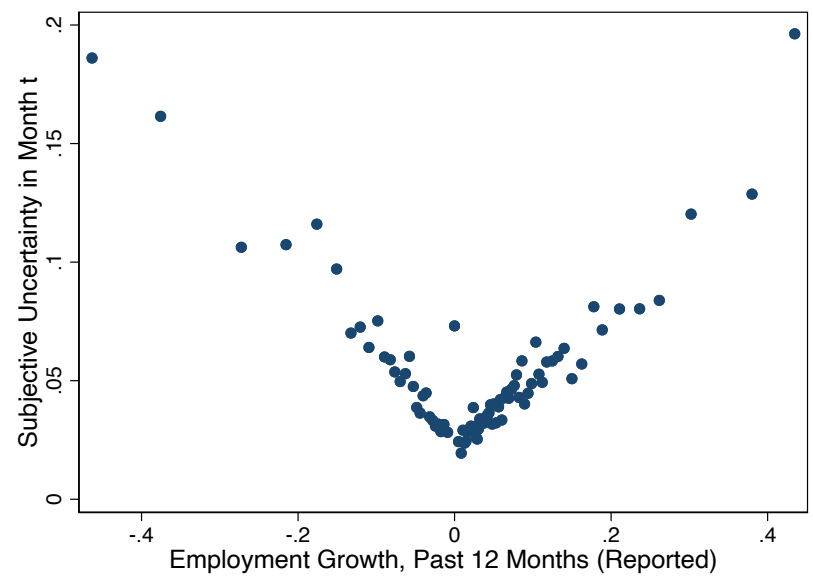

Figure C.5b

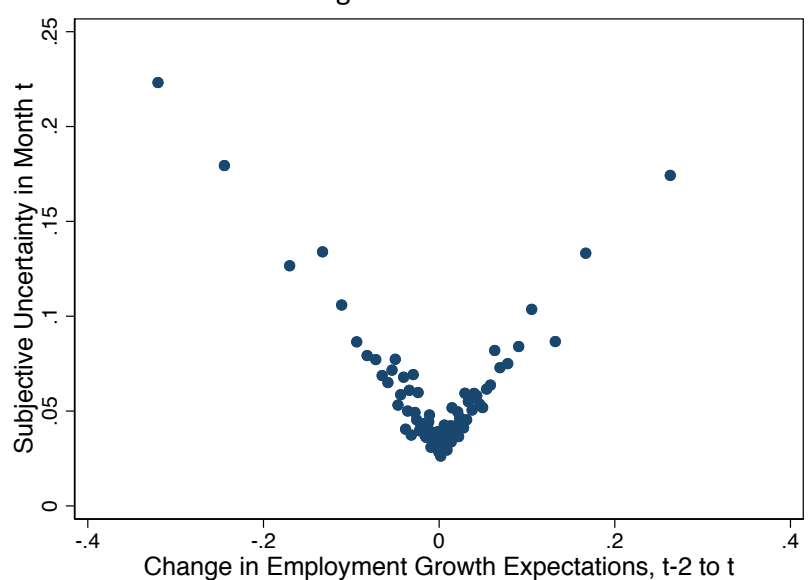

Notes: This figure shows two bin-scatter plots with subjective uncertainty over employment growth in the 12 months following month $t$ on the vertical axis. Figure $6 a$ shows shows 100 quantiles of past employment growth from month $t-12$ to $t$ on the horizontal axis. Figure $6 \mathrm{~b}$ instead shows 100 quantiles of the change in twelve-months-ahead employment growth expectations from $t-2$ (or $t-3$ ) to $t$. Data are from the SBU and the sample covers all survey waves from 10/2014 to 10/2019.

\section{Figure C.6: Reinterpreting responses as continuous distributions consisting of 5 uniform bins}

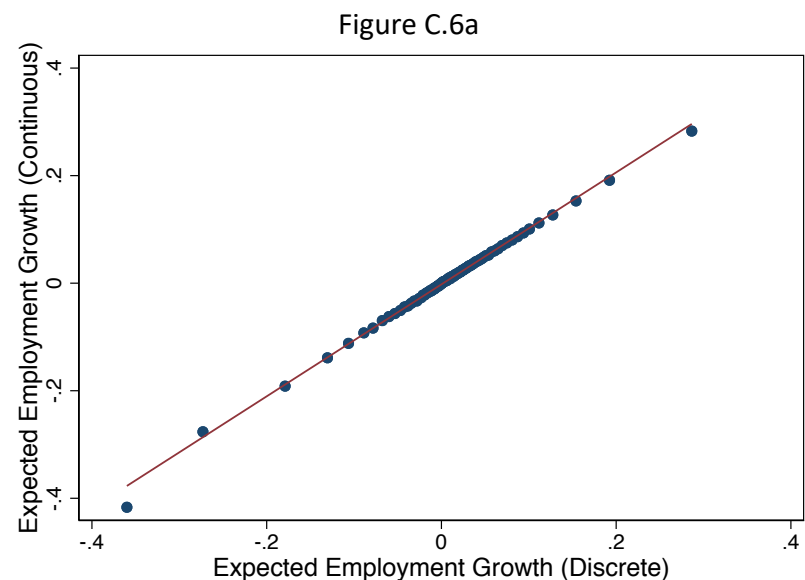

Coeff. $=1.04$, S.E. $=.005, \mathrm{R}^{\wedge} 2=.985, \mathrm{~N}=7064$

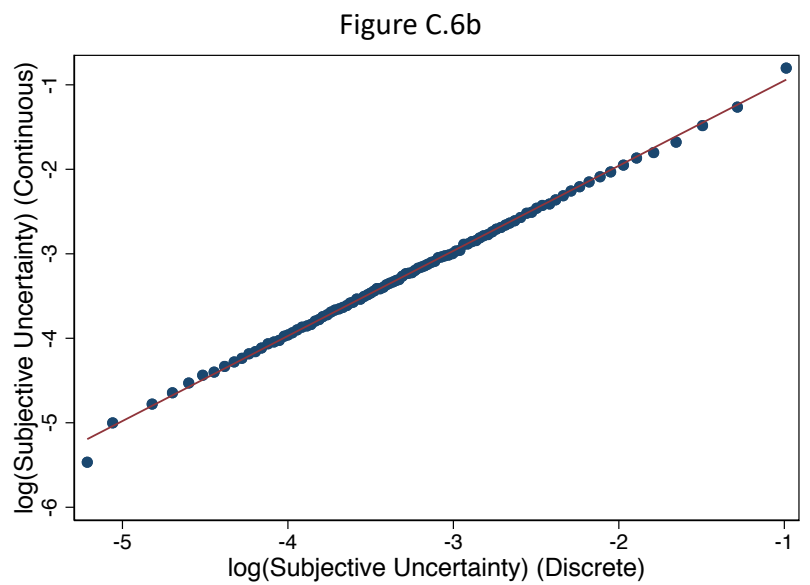

Coeff. $=1.00$, S.E. $=.004, \mathrm{R}^{\wedge} 2=.985, \mathrm{~N}=7070$

Notes: The above figures show bin-scatter plots that compare our measures of subjective mean expectations and uncertainty interpreting SBU responses as discrete or subjective distributions. Our baseline measures interpret SBU responses as discrete, 5-point probability distributions. Alternatively, we can interpret the responses as a continuous distribution consisting of 5 bins, with a uniform distribution within each bin. Figure 12a plots 100 percentiles of our discrete measure of expected employment growth (looking 12 months ahead) on the horizontal axis against the continuous measure of expectations on the vertical axis. Figure $12 \mathrm{~b}$ repeats the exercise for the natural logarithm of subjective uncertainty. Statistics below the figure correspond to the OLS regression in the underlying microdata, reporting firm-clustered standard errors. Data are from all waves of the SBU from 10/2014 to 10/2019. 


\section{Figure C.7: Subjective Uncertainty and Previous Survey Completions \\ Figure C.7a}

Dep Variable: log(Empl. Growth Uncertainty)

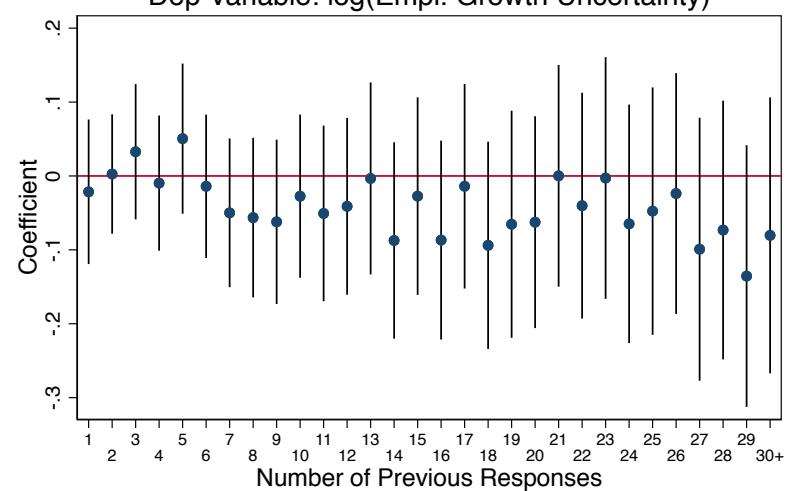

Mean (SD) of Dep. Var: -3.24 (.833)

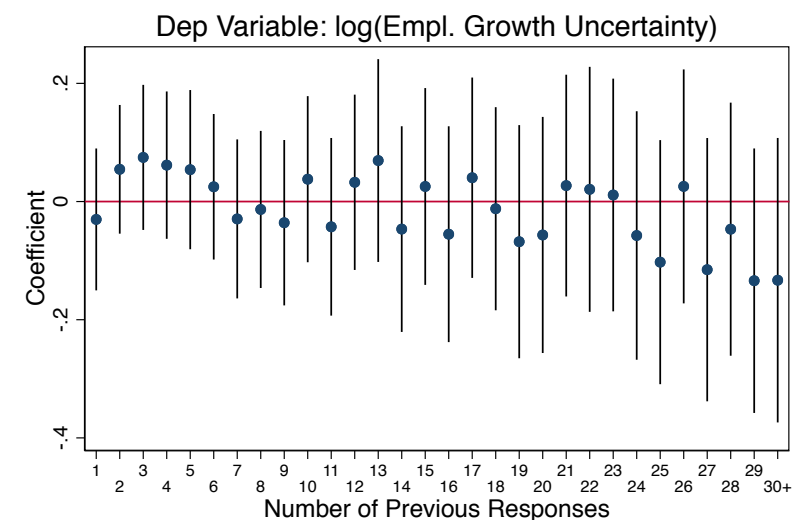

Mean (SD) of Dep. Var: -3.24 (.830). Employment-weighted

Notes: This figure shows estimated coefficients and 95 percent confidence intervals from regressions of the natural log of employment growth uncertainty (looking ahead over the next 4 quarters) on a set of indicators for the firm's number of previous SBU responses on the right-handside as well as firm and time fixed effects (not shown). Figure B.3a (left) shows unweighted estimates, while figure B.3b (right) weights observations by employment (winsorized at 500 employees). We top-code the number of responses at 30. Data are from the SBU and cover all survey waves between 10/2014 and 10/2019. We construct the 95 percent confidence intervals based on firm-clustered robust standard errors.

\section{C.2 Additional descriptive results about the SBU microdata}

- Below we report some additional results pertaining to our expectations and uncertainty indices based on the SBU data. 


\section{Figure C.8: Marginal Distributions C.8a. Reported Sales Growth Rates, Past 12 Months}

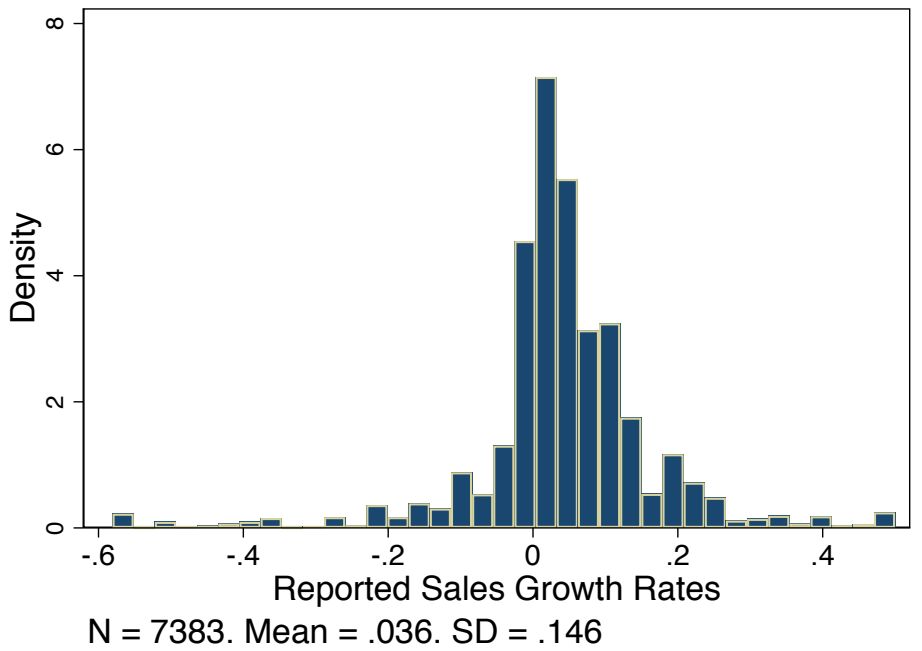

\section{C.8b. Sales Growth Expectations, Next 4 Quarters}

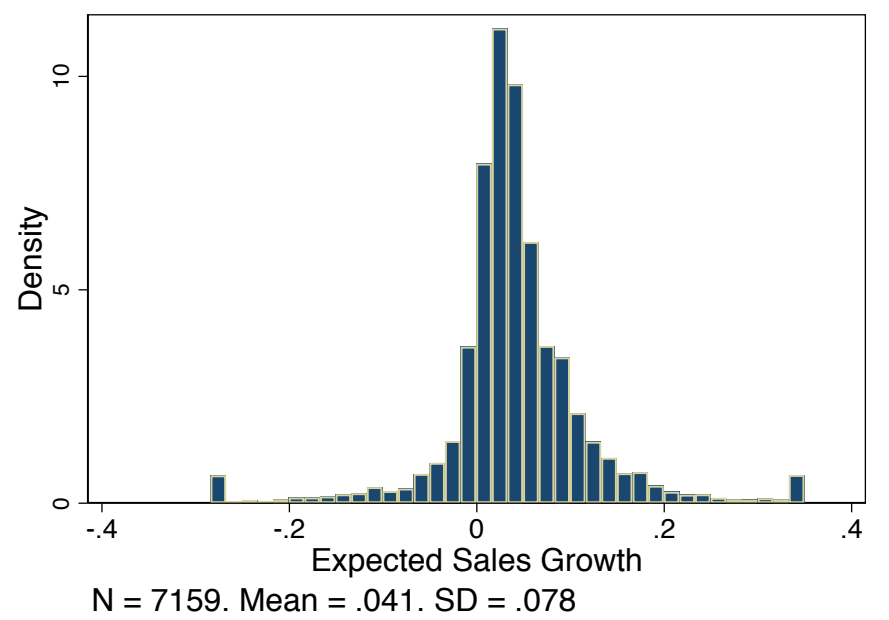

Notes: The histogram shows the empirical distribution of expected sales growth rates, looking ahead to the next four quarters. The sample includes all SBU responses between $10 / 2014$ to $10 / 2019$ for which we have a five-point subjective distribution over future employment growth rates. We compute these subjective mean growth rates as described in Section 2 of the main text. 


\section{C.8c. Sales Growth Uncertainty, Next 4 Quarters}

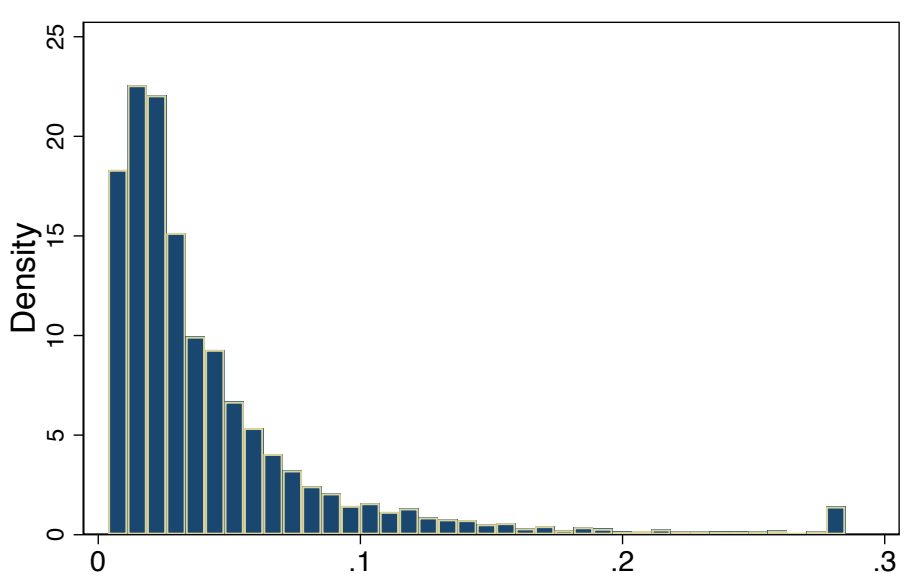

Subjective Uncertainty over Sales Growth $\mathrm{N}=7160$. Mean $=.044 . \mathrm{SD}=.047$
Notes: The histogram shows the empirical distribution of subjective uncertainty about sales growth, looking ahead to the next four quarters. The sample includes all SBU responses between 10/2014 to $10 / 2019$ for which we have a fivepoint subjective distribution over future sales growth rates. We compute subjective uncertainty about sales growth as the standard deviation of the five-point subjective distribution. See Section 2 of the main text for details.

\section{Figure C.9 Subjective uncertainty in month $t$ predicts the extent of beliefs revisions in the next survey}

\section{C.9a. Sales growth subjective distribution}

Figure C.9a.i Revising the Vector of Support Points

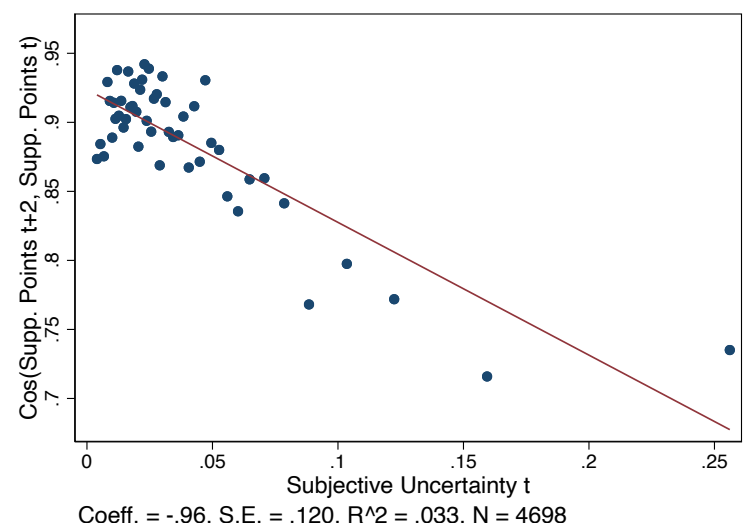

Figure C.9a.ii Revising the Vector of Probabilities

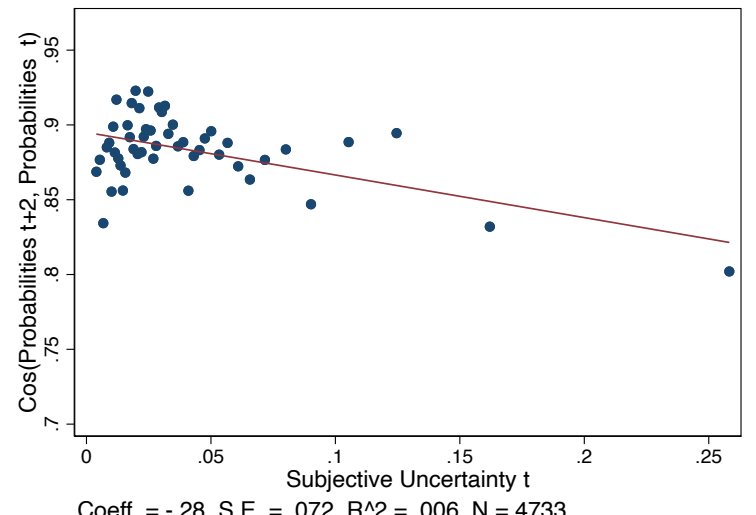

Notes: Both bin scatters show 50 quantiles of subjective uncertainty at $t$ for the sales growth rate over the next four quarters. The vertical axis in panel (a) shows the cosine similarity between forecast distribution support points for sales growth rates at $t$ and $t+2$ (or $t+3$ ) for the same firm. The vertical scale in panel (b) shows cosine similarity for forecast distribution probabilities at $t$ and $t+2$ (or $t+3$ ). We report the underlying firm-level regressions with firm-clustered standard errors at the bottom of each figure, using SBU data from 10/2014 to 10/2019. 


\section{C.9b Employment growth subjective distribution}

Figure C.9b.i Revising the Vector of Support Points

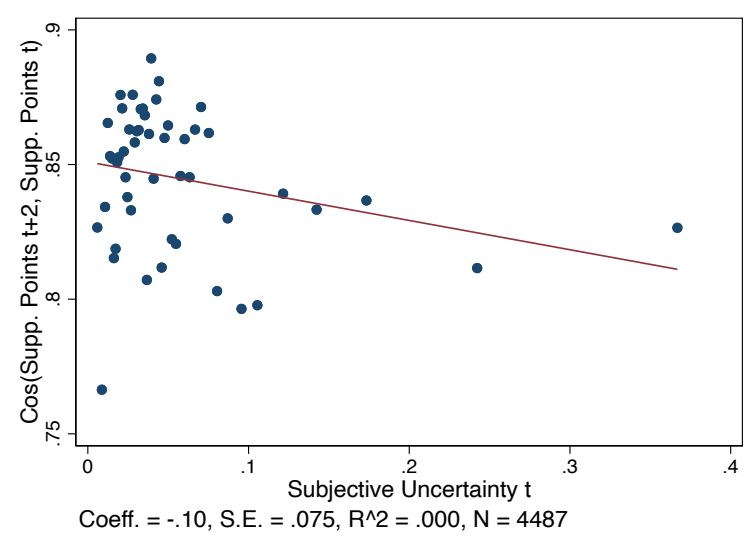

Figure C.9b.ii Revising the Vector of Probabilities

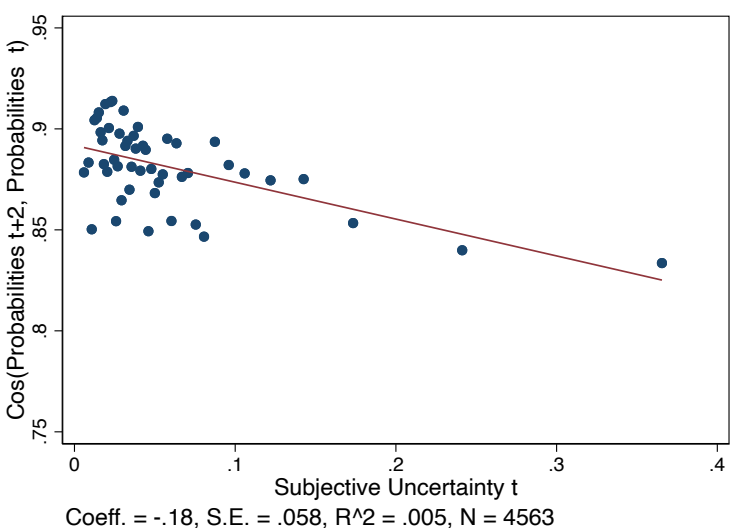

Notes: This figure shows two bin-scatter plots. On the horizontal axis, both show 50 quantiles of subjective uncertainty for employment growth over the next 12 months, measured in month $t$. In figure 5 a (left) the vertical axis shows the cosine similarity of the vector of support points respondents provide across consecutive surveys, in months $t$ and $t+2$ (or $t+3$ ). In figure $5 \mathrm{~b}$ (right) we instead show the cosine similarity across vectors of probabilities from nearest same-topic surveys. The regression results reported below each figure correspond to the underlying microdata regression, reporting firm-clustered standard errors. Data are from the SBU and the sample covers all survey waves from $10 / 2014$ to $10 / 2019$.

\section{Figure C.10: Predictive power of discrete vs. continuous subjective moments} C.10a. Sales Growth Expectations and Uncertainty

Figure C.10a.i

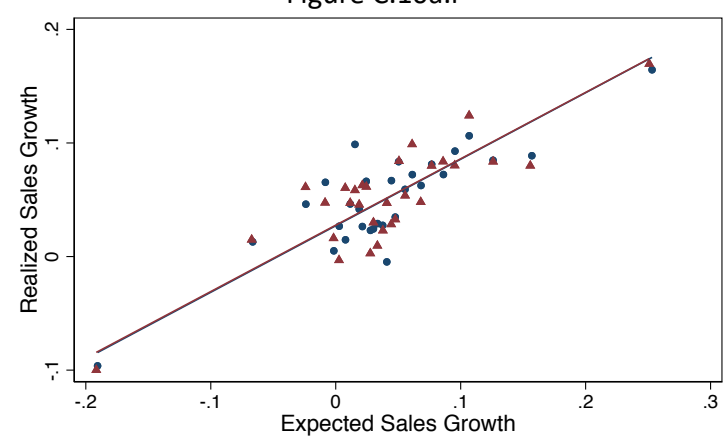

- Discrete Interpretation $\triangle$ Continuous Interpretation Discrete: .585 (.084). Continuous: .584 (.084). N = 6074

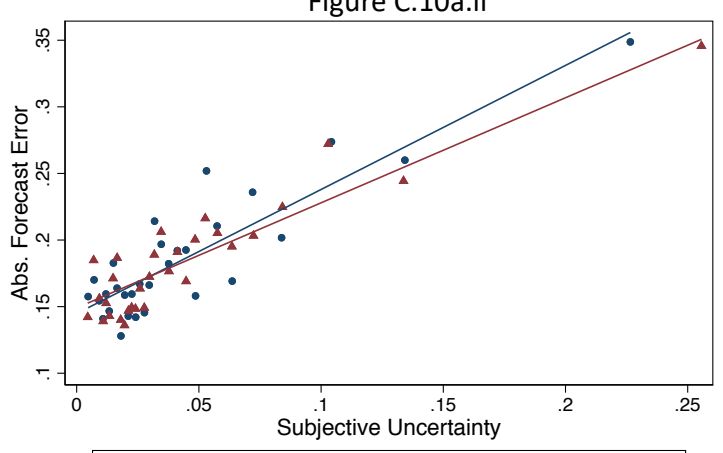

- Discrete Interpretation $\triangle$ Continuous Interpretation Discrete: .931 (.100). Continuous: .788 (.090). N = 6074

Notes: Each of the above figures figure superimposes two bin-scatter plots. On the left we show forecast employment growth over the next twelve months on the horizontal axis against actual employment growth. On the right we have subjective uncertainty over employment growth for the next twelve months on the horizontal axis against the respondent's absolute forecast error for employment growth over the ensuing twelve months on the vertical axis. The blue dots show each relationship under our baseline interpretation that SBU responses are discrete, 5-point probability distributions. The red triangles show the relationship if we interpret the responses as a continuous distribution consisting of 5 bins, with a uniform distribution within each bin. Statistics below the figure correspond to the population OLS regression, reporting firm-clustered standard errors. Data are from all waves of the SBU from 10/2014 to 10/2019. 


\section{C.10b. Employment Growth Expectations and Uncertainty}
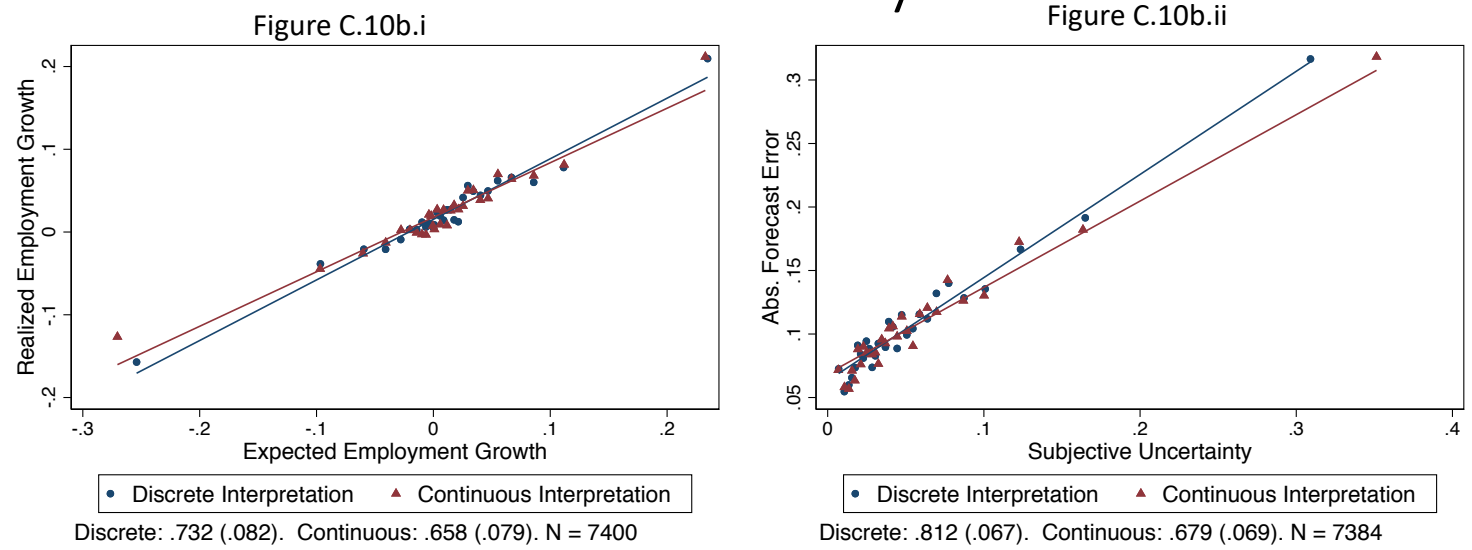

Notes: Each of the above figures figure superimposes two bin-scatter plots. On the left we show forecast employment growth over the next twelve months on the horizontal axis against actual employment growth. On the right we have subjective uncertainty over employment growth for the next twelve months on the horizontal axis against the respondent's absolute forecast error for employment growth over the ensuing twelve months on the vertical axis. The blue dots show each relationship under our baseline interpretation that SBU responses are discrete, 5-point probability distributions. The red triangles show the relationship if we interpret the responses as a continuous distribution consisting of 5 bins, with a uniform distribution within each bin. Statistics below the figure correspond to the population OLS regression, reporting firm-clustered standard errors. Data are from all waves of the SBU from 10/2014 to 10/2019.

\section{Figure C.11: Uncertainty vs. Firm Size}

Figure C.11a

Sales Growth Uncertainty vs. Size

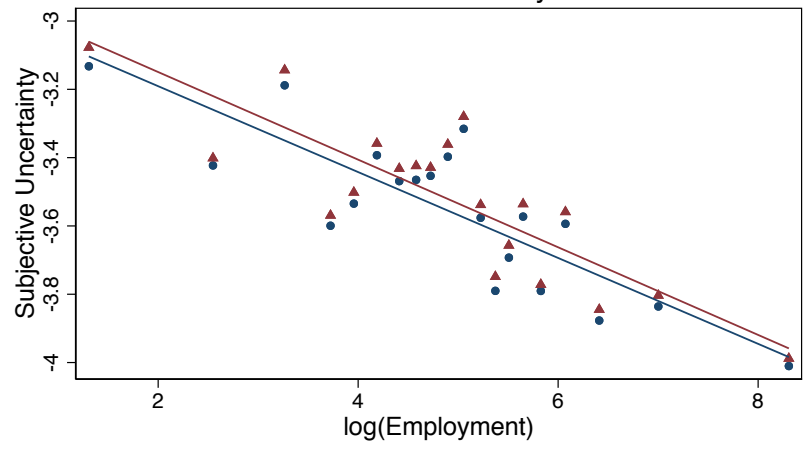

- Discrete Interpretation $\Delta$ Continuous Interpretation

Discrete: - .12 (.020). Continuous: -.12 (.021). $N=6882$
Figure C.11b

Employment Growth Uncertainty vs. Size

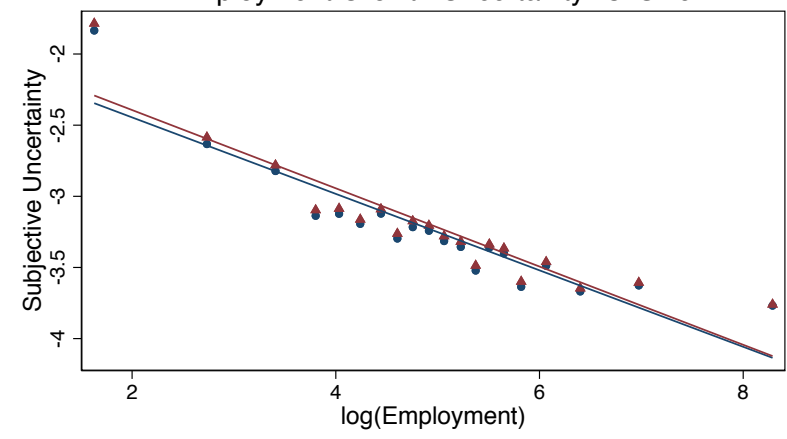

- Discrete Interpretation $\Delta$ Continuous Interpretation

Discrete: -.26 (.019). Continuous: -.27 (.020). N = 7073

Notes: Each of the above figures shows a bin-scatter plot of the natural logarithm of subjective uncertainty on the vertical axis against 20 quantiles of the natural log of current firm-level employment. Figure B.11a focuses on sales growth uncertainty for the next four quarters on the vertical axis, while Figure B.11b focuses on employment growth uncertainty looking ahead over the next twelve months. Subjective uncertainty is the standard deviation of each respondents' five-point subjective distribution over future sales or employment growth. See Section 2 and Appendix A for more details on how we measure subjective uncertainty. The statistics below each figure report the slope coefficient and firm-clustered standard error in the underlying microdata regression. Data are from all waves of the SBU from 10/2014 to 10/2019. 


\section{Figure C.12: Uncertainty vs. Age C.12a. Without controlling for size}

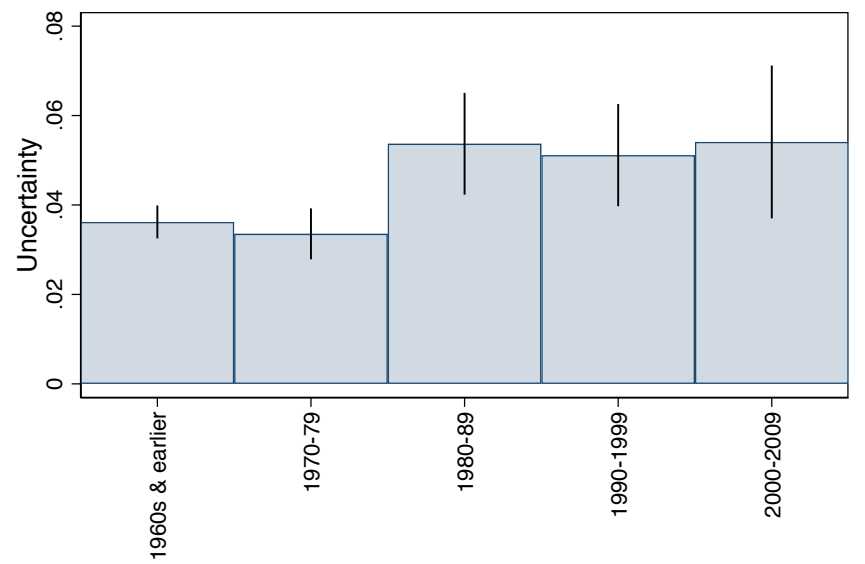

Year Hired 1st Employee
Notes: This figure shows within group means and 95 percent confidence intervals of sales growth uncertainty looking ahead over the next four quarters, grouping firms by the decade in which they hired their first paid employee. Data are from the SBU and cover all survey waves between $10 / 2014$ and 10/2019. The vertical lines are 95 percent confidence intervals based on firm clustered robust standard errors.

\section{C.12b. Controlling for Size}

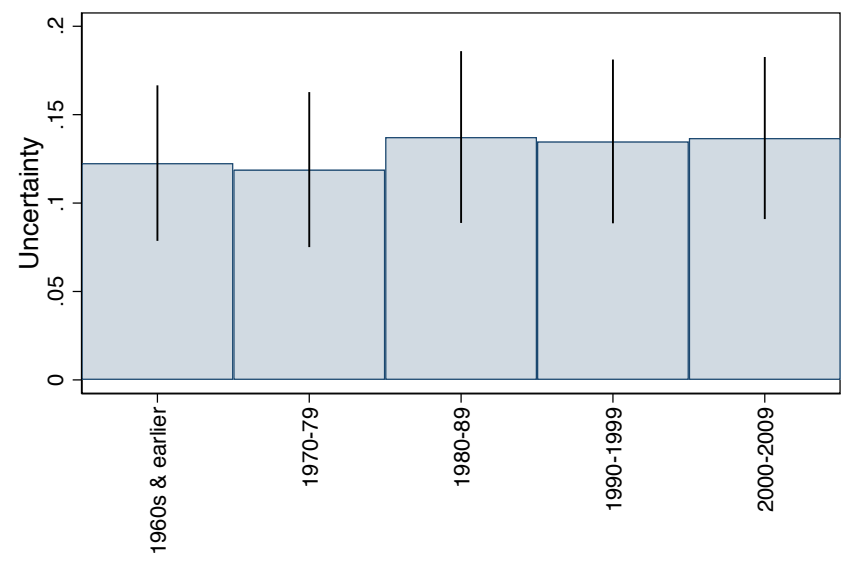

Year Hired 1st Employee
Notes: This figure shows within group means and 95 percent confidence intervals of sales growth uncertainty over the next four quarters, grouping firms by the decade in which they hired their first paid employee after controlling for the relationship between uncertainty and firm size (measured as log(current sales)). Data are from the SBU and cover all survey waves between 10/2014 and 10/2019. The vertical lines are 95 percent confidence intervals based on firm clustered robust standard errors. 


\section{Table C.1: How do higher-order subjective moments predict outcomes and forecast errors?}

C.1a. Sales Growth, Next 4 Quarters

\begin{tabular}{|c|c|c|c|c|c|c|c|c|}
\hline \multirow{3}{*}{$\begin{array}{l}\text { Dependent Variable } \\
\text { Expected Sales Growth, Next } 4 \text { Quarters }\end{array}$} & (1) & (2) & (3) & (4) & & (6) & $(7)$ & $(8)$ \\
\hline & \multicolumn{4}{|c|}{ Realized Sales Growth, Next 4 Quarters } & \multicolumn{4}{|c|}{$\begin{array}{c}\text { Abs(Forecast - Realized Sales Growth), } \\
\text { Next } 4 \text { Quarters }\end{array}$} \\
\hline & $\begin{array}{c}0.589 * * * \\
(0.084)\end{array}$ & $\begin{array}{c}0.569 * * * \\
(0.081)\end{array}$ & $\begin{array}{c}0.569 * * * \\
(0.081)\end{array}$ & $\begin{array}{c}0.451 * * * \\
(0.110)\end{array}$ & $\begin{array}{c}0.032 \\
(0.060)\end{array}$ & $\begin{array}{c}0.006 \\
(0.061)\end{array}$ & $\begin{array}{c}0.006 \\
(0.061)\end{array}$ & $\begin{array}{l}-0.025 \\
(0.059)\end{array}$ \\
\hline Sales Growth Uncertainty, Next 4 Quarters & $\begin{array}{c}0.058 \\
(0.163)\end{array}$ & $\begin{array}{c}0.087 \\
(0.159)\end{array}$ & $\begin{array}{c}0.095 \\
(0.155)\end{array}$ & $\begin{array}{c}0.065 \\
(0.229)\end{array}$ & $\begin{array}{c}0.936 * * * \\
(0.100)\end{array}$ & $\begin{array}{c}0.974 * * * \\
(0.098)\end{array}$ & $\begin{array}{c}0.948 * * * \\
(0.096)\end{array}$ & $\begin{array}{c}0.400 * * * \\
(0.150)\end{array}$ \\
\hline Sales Growth Skewness, Next 4 Quarters & & $\begin{array}{l}0.019 * * \\
(0.008)\end{array}$ & $\begin{array}{l}0.020 * * \\
(0.008)\end{array}$ & $\begin{array}{c}0.026 * * * \\
(0.008)\end{array}$ & & $\begin{array}{c}0.025 * * * \\
(0.005)\end{array}$ & $\begin{array}{c}0.024 * * * \\
(0.005)\end{array}$ & $\begin{array}{l}0.008^{*} \\
(0.004)\end{array}$ \\
\hline Sales Growth Kurtosis, Next 4 Quarters & & & $\begin{array}{l}-0.001 \\
(0.002)\end{array}$ & $\begin{array}{l}-0.002 \\
(0.002)\end{array}$ & & & $\begin{array}{c}0.002 \\
(0.001)\end{array}$ & $\begin{array}{c}0.000 \\
(0.001)\end{array}$ \\
\hline Time FE & & & & Y & & & & Y \\
\hline Firm FE & & & & $\mathrm{Y}$ & & & & $\mathrm{Y}$ \\
\hline Observations & 3,037 & 3,037 & 3,037 & 2,913 & 3,037 & 3,037 & 3,037 & 2,913 \\
\hline R-squared & 0.026 & 0.030 & 0.030 & 0.287 & 0.049 & 0.062 & 0.062 & 0.397 \\
\hline Adjusted R-squared & 0.0258 & 0.0293 & 0.0291 & 0.161 & 0.0480 & 0.0606 & 0.0612 & 0.290 \\
\hline Firms & 511 & 511 & 511 & 387 & 511 & 511 & 511 & 387 \\
\hline
\end{tabular}

\section{C.1b. Employment, Next 12 Months}

\begin{tabular}{|c|c|c|c|c|c|c|c|c|}
\hline \multirow{3}{*}{$\begin{array}{l}\text { Dependent Variable } \\
\text { Expected Employment Growth, Next } 12 \text { Months }\end{array}$} & (1) & (2) & (3) & (4) & \multirow{2}{*}{\multicolumn{4}{|c|}{$\begin{array}{ccc}(5) & (6) & (7) \\
\text { Abs(Forecast }- \text { Realized Employment Growth) } \\
\text { Next } 12 \text { Months }\end{array}$}} \\
\hline & \multicolumn{4}{|c|}{ Realized Employment Growth, Next 12 Months } & & & & \\
\hline & $\begin{array}{c}0.751 * * * \\
(0.083)\end{array}$ & $\begin{array}{c}0.732 * * * \\
(0.080)\end{array}$ & $\begin{array}{c}0.734^{* * *} \\
(0.079)\end{array}$ & $\begin{array}{c}0.671^{* * *} \\
(0.109)\end{array}$ & $\begin{array}{c}0.002 \\
(0.062)\end{array}$ & $\begin{array}{l}-0.006 \\
(0.059)\end{array}$ & $\begin{array}{c}-0.001 \\
(0.059)\end{array}$ & $\begin{array}{c}-0.142 * * \\
(0.059)\end{array}$ \\
\hline Employment Growth Uncertainty, Next 12 Months & $\begin{array}{c}0.114 \\
(0.082)\end{array}$ & $\begin{array}{c}0.126 \\
(0.082)\end{array}$ & $\begin{array}{c}0.118 \\
(0.083)\end{array}$ & $\begin{array}{c}0.143 \\
(0.134)\end{array}$ & $\begin{array}{c}0.813 * * * \\
(0.070)\end{array}$ & $\begin{array}{c}0.818^{* * *} \\
(0.071)\end{array}$ & $\begin{array}{c}0.795^{* * *} \\
(0.069)\end{array}$ & $\begin{array}{c}0.361^{* * *} \\
(0.097)\end{array}$ \\
\hline Employment Growth Skewness, Next 12 Months & & $\begin{array}{c}0.010 * * \\
(0.004)\end{array}$ & $\begin{array}{c}0.011 * * \\
(0.004)\end{array}$ & $\begin{array}{c}0.015^{* * *} * \\
(0.005)\end{array}$ & & $\begin{array}{c}0.004 \\
(0.004)\end{array}$ & $\begin{array}{l}0.006^{*} \\
(0.003)\end{array}$ & $\begin{array}{c}0.000 \\
(0.003)\end{array}$ \\
\hline Employment Growth Kurtosis, Next 12 Months & & & $\begin{array}{c}0.001 \\
(0.001)\end{array}$ & $\begin{array}{c}0.001 \\
(0.002)\end{array}$ & & & $\begin{array}{c}0.003 * * * \\
(0.001)\end{array}$ & $\begin{array}{c}0.001 \\
(0.001)\end{array}$ \\
\hline Time FE & & & & Y & & & & $\mathrm{Y}$ \\
\hline Firm FE & & & & Y & & & & $\mathrm{Y}$ \\
\hline Observations & 3,692 & 3,692 & 3,692 & 3,570 & 3,692 & 3,692 & 3,692 & 3,570 \\
\hline R-squared & 0.112 & 0.115 & 0.115 & 0.349 & 0.139 & 0.139 & 0.145 & 0.477 \\
\hline Adjusted R-squared & 0.112 & 0.114 & 0.114 & 0.242 & 0.138 & 0.139 & 0.144 & 0.391 \\
\hline Firms & 576 & 576 & 576 & 454 & 576 & 576 & 576 & 454 \\
\hline
\end{tabular}




\section{Additional Empirical Results: Aggregate Indices}

\section{Figure D.1: Investment Rate Indices}

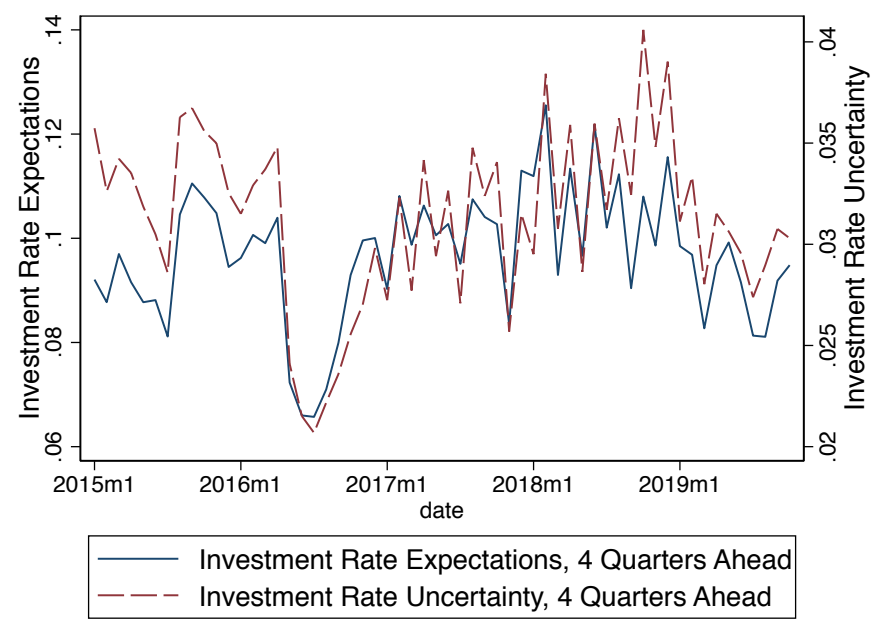

Notes: This figure shows our investment rate expectations (left axis) and uncertainty (right axis) indices, looking four quarters ahead. We smooth both indices using the same procedure as for the sales growth and employment growth indices, which we describe in the main text. Both indices appear on the official Survey of Business Uncertainty website as of February 2020. 


\section{Figure D.2: Employment growth expectations and uncertainty Indices controlling for panel composition}

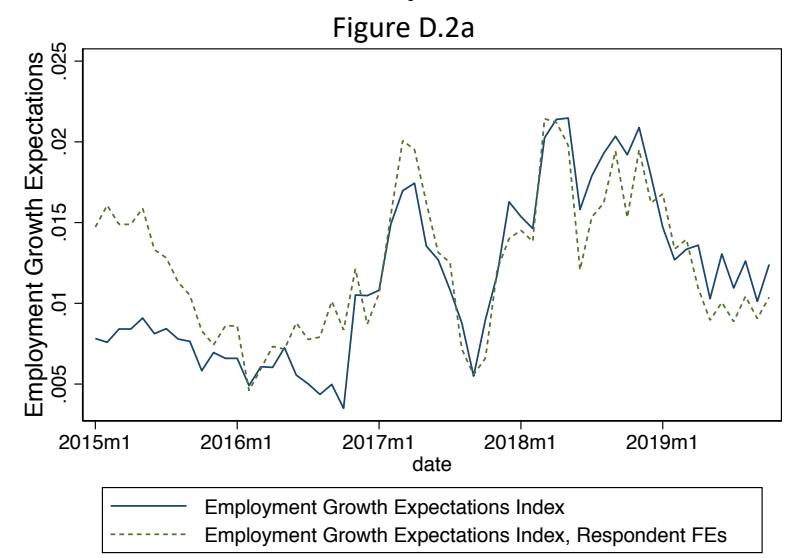

Correlation .806

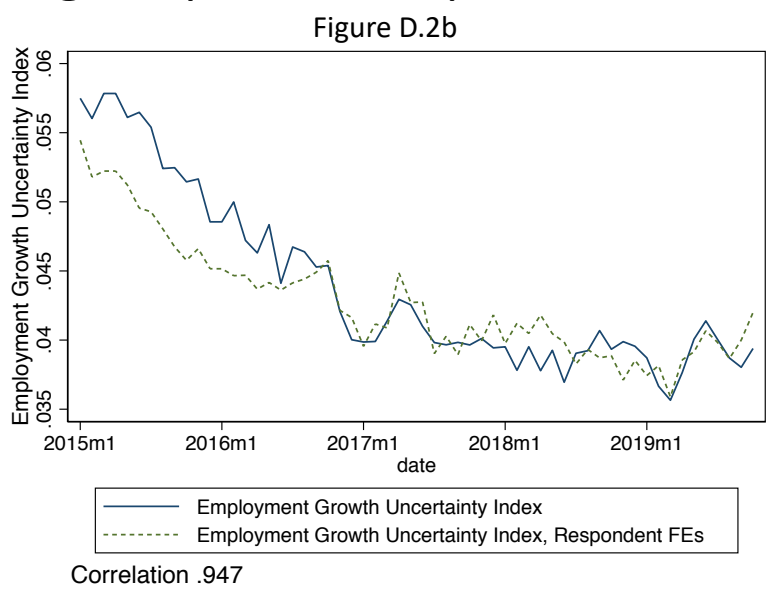

Notes: The above figures plot our baseline employment growth expectations (left) and uncertainty (right) indices alongside an alternative index that accounts for changing panel composition across months. Our baseline index computes an activity-weighted mean for expectations or uncertainty in each month. By contrast, the alternative index computes the same activity weighted mean after controlling for respondent fixed effects. We smooth both indices using the same procedure. Data are from the SBU and cover all months between 1/2015 and 10/2019.

\section{Figure D.3: Our sales growth expectations and uncertainty indices and 95 percent confidence bands with/without controlling for panel composition}

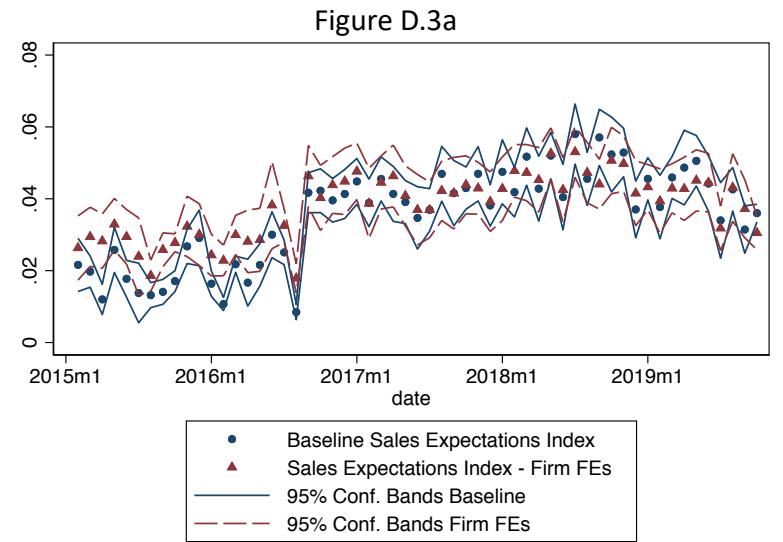

Correlation .942

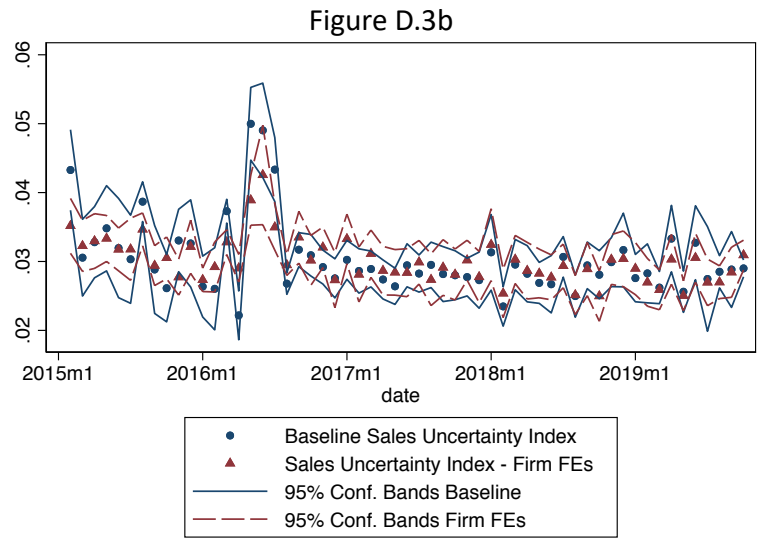

Correlation .854

Notes: The above figures plot our baseline sales growth expectations (left) and uncertainty (right) indices alongside alternative indices that account for changing panel composition across months and also display 95 percent confidence bands for each index based on two-way firm and date clustered robust standard errors. Our baseline index computes an activity-weighted mean for expectations or uncertainty in each month. By contrast, the alternative index computes the same activity weighted mean controlling for respondent fixed effects. This figure does not smooth the indices time series. Data are from the SBU and cover all months between 1/2015 and 10/2019. 


\section{D.1 How do our expectations and uncertainty indices change if we reweight our sample to match the share of employment by industry and region?}

- In the following pages, we test how reweighting our SBU sample to resemble its target population (the private nonfarm sector) more closely changes our expectations and uncertainty indices.

- We construct versions of our indices that match the share of employment by industry and region in each year, and compare them with our baseline (employment-weighted) indices.

- We obtain the target industry and region employment shares using private nonfarm payroll data from the Bureau of Labor Statistics.

\section{D.1.1 Reweighting Procedure}

1. We calculate industry employment shares (source: BLS) for each year from 2014 to 2018. Data for 2019 are not yet available as of mid-March 2020, so we use the 2018 shares for 2019. Let $P O P_{j t}$ denote industry j's employment share in year $t$.

2. We calculate region employment shares (source: BLS) in each year from 2014 to 2018. Data for 2019 are not yet available as of mid-March 2020, so we use the 2018 shares for 2019. Let $P O P_{r t}$ denote region $r$ 's employment share in year $t$.

3. We calculate industry and region employment shares in the SBU for each year from 2014 to 2019 , based on reported employment (winsorized at 500 employees). Let $S B U_{j t}$ denote industry j's employment share in year $t$, and $S B U_{r t}$ region $r$ 's employment share in year $t$ in the SBU.

4. We calculate industry (region) representativeness weights by dividing the population industry (region) employment share by its SBU counterpart $P O P_{j t} / S B U_{j t}\left(P O P_{r t} / S B U_{r t}\right)$.

5. We then multiply our baseline activity weight--the firm's employment, winsorized at $500--b y$ the appropriate industry and region representativeness weights, i.e the product $P O P_{j t} / S B U_{j t} \times P O P_{r t} / S B U_{r t}$.

6. We use the adjusted weights from step 5 to construct topic-specific indices for sales growth and employment, following the procedure described in the main text. 


\section{D.1.2 Industry and Region Disaggregation}

- The industry disaggregation we consider is the following:

- 1) Construction; 2) Durable goods manufacturing; 3) Educational services; 4) Finance and insurance; 5) Health care and social assistance; 6) Information; 7) Leisure and hospitality; 8) Mining and utilities; 9)Nondurable goods manufacturing; 10) Other services except government; 11) Professional and business services; 12) Real estate and rental and leasing 13) Retail and wholesale trade 14) Transportation and warehousing

- The regional disaggregation we consider uses the nine Census Divisions:

- 1) New England; 2) Mid-Atlantic; 3) East North Central; 4) West North Central;

5) South Atlantic; 6) East South Central; 7) West South Central; 8) Mountain;

9) Pacific

\section{Figure D.4: Sales growth rate indices, baseline and reweighted to match industry and region employment shares, no smoothing}

Figure D.4a

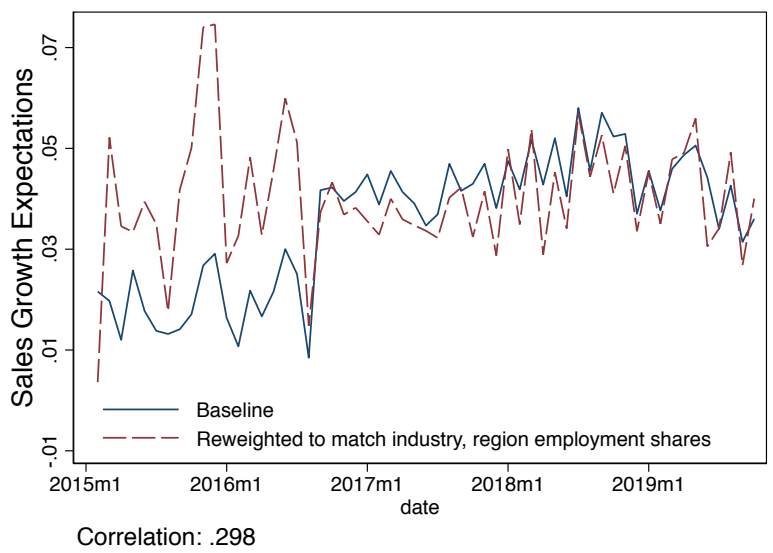

Figure D.4b

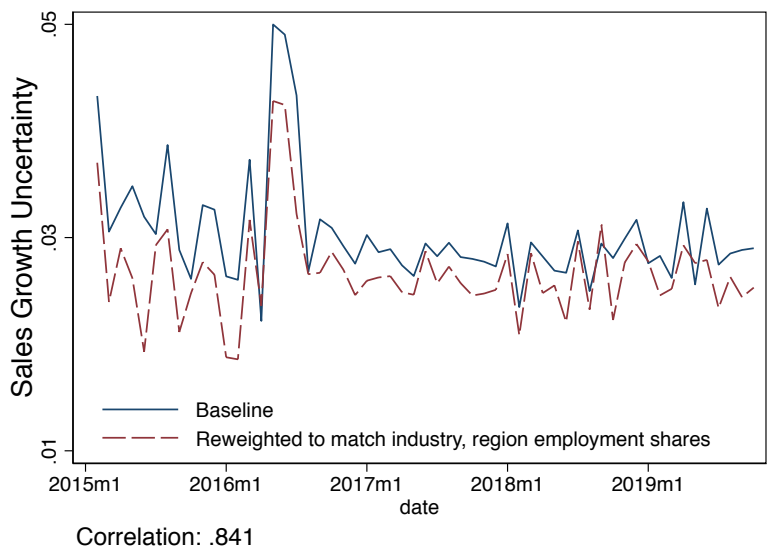

Notes: The above figures plot our baseline employment-weighted sales growth expectations (left) and uncertainty (right) indices, respectively alongside alternative indices that reweight the microdata to match the employment shares of 14 major industries and 9 Census Divisions by year in BLS nonfarm payrolls data. This figure does not smooth the baseline or reweighted indices. Data are from the SBU and BLS and cover all months between 1/2015 and 10/2019. 


\section{Figure D.5: Sales growth rate indices, baseline and reweighted to match industry and region employment shares, smoothed}
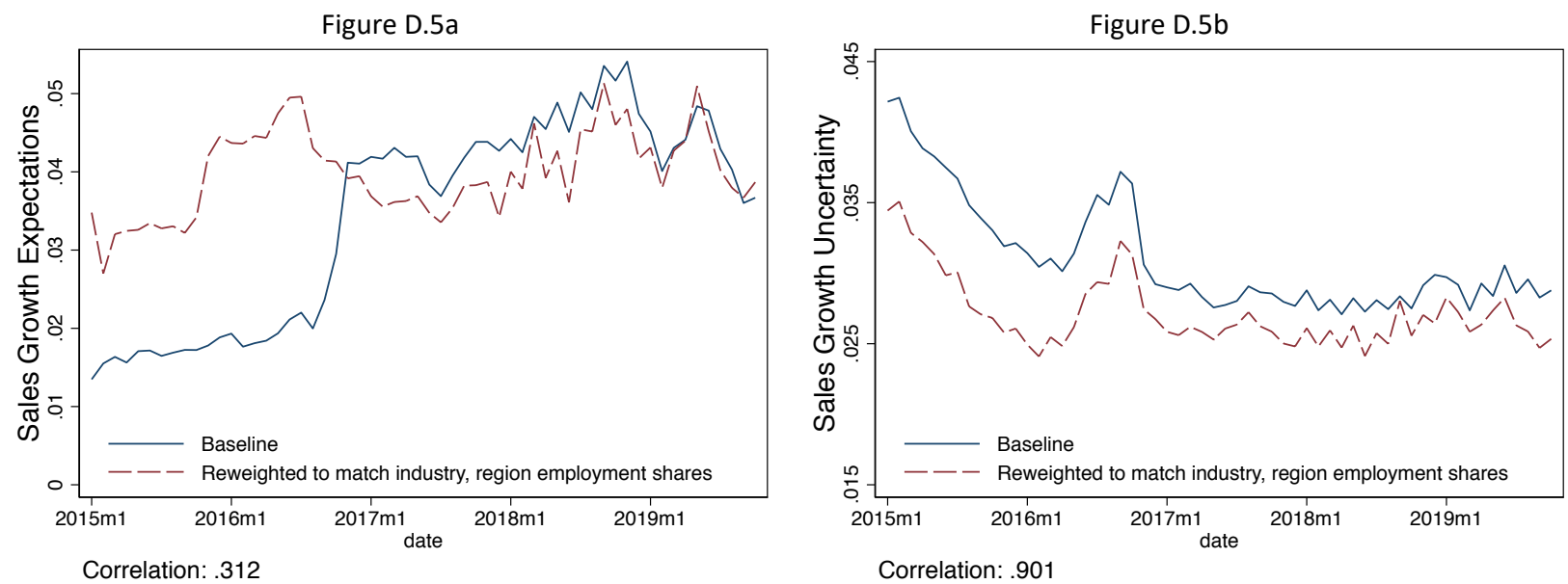

Notes: The above figures plot our baseline employment-weighted sales growth expectations (left) and uncertainty (right) indices, respectively alongside alternative indices that that reweight the microdata to match the employment shares of 14 major industries and 9 Census Divisions by year in BLS nonfarm payrolls data. This figure smooths the baseline and reweighted indices using the procedure outlined in the main text. Data are from the SBU and BLS and cover all months between 1/2015 and 10/2019.

\section{Figure D.6: Employment growth indices, baseline and reweighted to match industry and region employment shares, no smoothing}
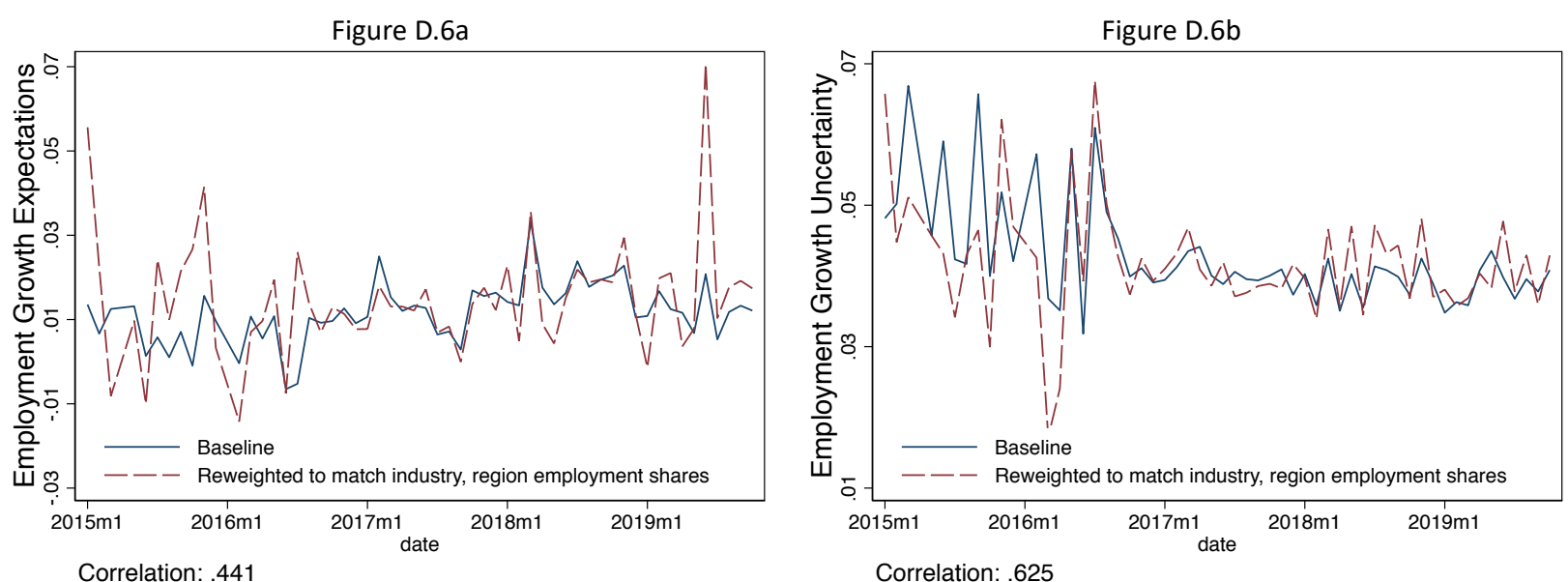

Notes: The above figures plot our baseline employment-weighted employment growth expectations (left) and uncertainty (right) indices, respectively alongside alternative indices that reweight the microdata to match the employment shares of 14 major industries and 9 Census Divisions by year in BLS nonfarm payrolls data. This figure does not smooth the baseline or reweighted indices. Data are from the SBU and BLS and cover all months between 1/2015 and 10/2019. 


\section{Figure D.7: Employment growth indices, baseline and reweighted to match industry and region employment shares, smoothed}
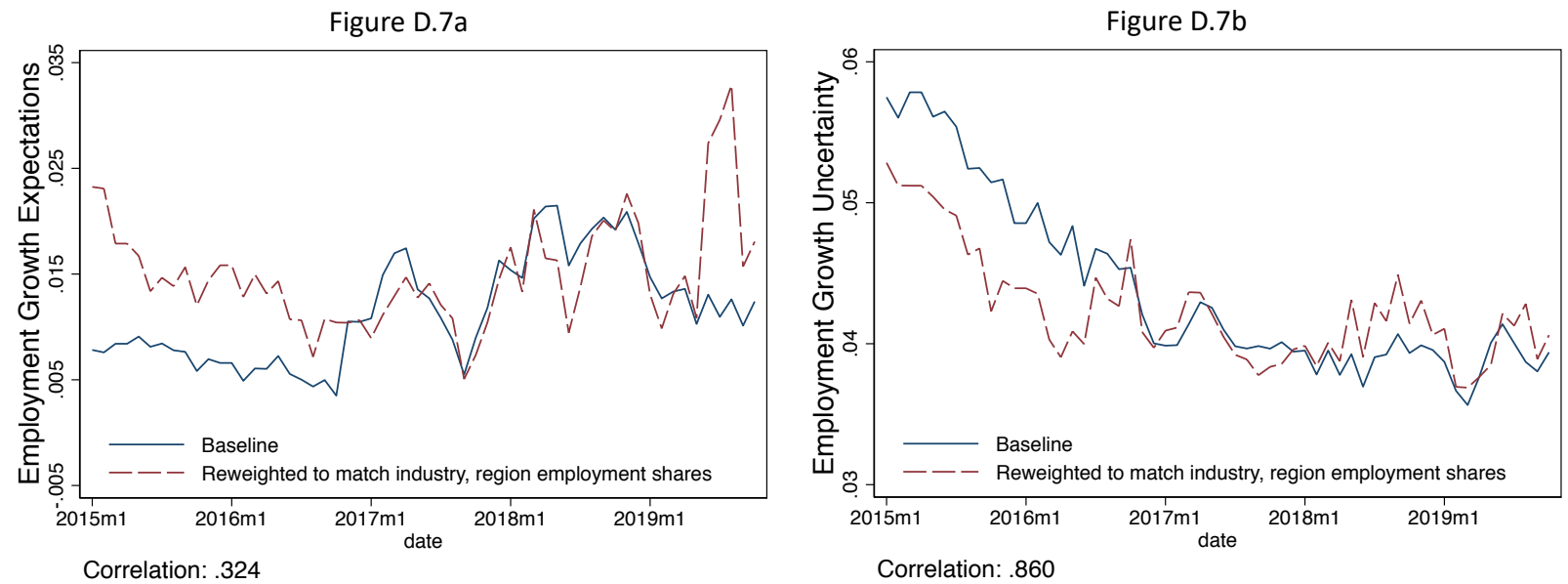

Notes: The above figures plot our baseline employment-weighted employment growth expectations (left) and uncertainty (right) indices, respectively alongside alternative indices that reweight the microdata to match the employment shares of 14 major industries and 9 Census Divisions by year in BLS nonfarm payrolls data. This figure smooths the baseline and reweighted indices using the procedure outlined in the main text. Data are from the SBU and BLS and cover all months between 1/2015 and 10/2019. 


\section{Table D.1 Summary Statistics for Baseline and Reweighted indices, 9/2016 to 10/2019}

\section{Table D.1a Sales growth rate indices}

\begin{tabular}{ccccccc}
\hline Moment & $\begin{array}{c}\text { Reweighted to } \\
\text { match empl. shares }\end{array}$ & Smoothing? & $\begin{array}{c}\text { Corr(Baseline, } \\
\text { Reweighted) } \\
\text { (\%) }\end{array}$ & $\begin{array}{c}\text { SD(Baseline) } \\
\text { (\%) }\end{array}$ & $\begin{array}{c}\text { SD(Reweighted) } \\
\text { (\%) }\end{array}$ & $\begin{array}{c}\text { Mean(Baseline } \\
\text { - Reweighted) } \\
\text { (\%) }\end{array}$ \\
\hline Expectations & Industry, region & No & 79.4 & 0.624 & 0.803 & 0.350 \\
Uncertainty & Industry, region & No & 71.1 & 0.206 & 0.228 & 0.251 \\
Expectations & Industry, region & Yes & 56.7 & 0.587 & 0.451 & 0.269 \\
Uncertainty & Industry, region & Yes & 90.1 & 0.206 & 0.163 & 0.256 \\
Expectations & Industry & No & 81.8 & 0.624 & 0.716 & 0.336 \\
Uncertainty & Industry & No & 79.9 & 0.206 & 0.207 & 0.229 \\
Expectations & Industry & Yes & 86.6 & 0.587 & 0.499 & 0.308 \\
Uncertainty & Industry & Yes & 90.7 & 0.206 & 0.146 & 0.240 \\
\hline
\end{tabular}

\section{Table D.1b Employment growth rate indices}

\begin{tabular}{lcccccc}
\hline Moment6 & $\begin{array}{c}\text { Reweighted to match } \\
\text { empl. shares }\end{array}$ & Smoothing? & $\begin{array}{c}\text { Corr(Baseline } \\
\text { Reweighted) } \\
\text { (\%) }\end{array}$ & $\begin{array}{c}\text { SD(Baseline) } \\
\text { (\%) }\end{array}$ & $\begin{array}{c}\text { SD(Reweighted) } \\
\text { (\%) }\end{array}$ & $\begin{array}{c}\text { Mean(Baseline } \\
\text { - Reweighted) } \\
\text { (\%) }\end{array}$ \\
\hline Expectations & Industry, region & No & 61.0 & 0.597 & 1.196 & -0.096 \\
Uncertainty & Industry, region & No & 61.4 & 0.254 & 0.395 & -0.080 \\
Expectations & Industry, region & Yes & 41.3 & 0.456 & 0.595 & -0.116 \\
Uncertainty & Industry, region & Yes & 68.6 & 0.199 & 0.230 & -0.083 \\
Expectations & Industry & No & 72.8 & 0.597 & 0.907 & 0.021 \\
Uncertainty & Industry & No & 70.3 & 0.254 & 0.356 & -0.082 \\
Expectations & Industry & Yes & 68.6 & 0.456 & 0.424 & 0.006 \\
Uncertainty & Industry & Yes & 72.7 & 0.199 & 0.210 & -0.075 \\
\hline
\end{tabular}

Notes: This table reports the correlation and individual standard deviations of our baseline and reweighted expectations and uncertainty indices. The top panel (Table D.1a) focuses on sales growth rate indices while the bottom panel (Table D.1b) focuses on employment growth rate indices. We report those statistics separately for both raw and smoothed versions of the indices. In the latter case we use the smoothing procedure described in the main text. We report statistics for reweighted indices that match both industry and region employment shares, where the reweighting follows the procedure in section D.1.1 above, as well as indices where we reweight to match only industry employment shares. Data are from the SBU and the sample period covers $9 / 2016$ to $10 / 2019$. 